\title{
Electrotransfection of polyamine folded DNA origami structures
}

\author{
Aradhana Chopra ${ }^{1}$, Swati Krishnan ${ }^{1}$ and Friedrich C. Simmel ${ }^{1 *}$ \\ ${ }^{1}$ Physik-Department E14, Technische Universität München, 85748 Garching, Germany \\ Email: simmel@tum.de, Phone: +49 (0)89 289 11611, Fax: +49 (0)89 28911612.
}

Supporting Information consisting of

- Experimental section

- Supporting figures S1-S16

- Supporting tables 1-3

- Supporting Videos 1-2 


\section{Experimental section}

Assembly of DNA origami structures. Three different DNA origami nanostructures - the twist corrected regular Rothemund rectangle (tCRRO), a single-layered square lattice structure consisting of 24 parallel double helices with dimensions $90 \mathrm{~nm} \times 65 \mathrm{~nm} \times 2 \mathrm{~nm}$ (length $\times$ width $\times$ height $)^{1,2}$, a DNA origami tube (a hollow honeycomb lattice structure with dimensions $83 \mathrm{~nm}$ $\times 23 \mathrm{~nm} \times 29 \mathrm{~nm}$ (length $\times$ diameter $1 \times$ diameter 2$)$ ), and a 42 helix bundle $(42 \mathrm{HB}$, a compact honeycomb lattice structure with dimensions $60 \mathrm{~nm} \times 26 \mathrm{~nm} \times 16 \mathrm{~nm}^{3}$ - were each assembled in one pot reactions using different variants of M13mp18 scaffold and their respective sets of DNA staple strands (used at $3 \mathrm{x}$ excess) in a variety of folding buffers - traditional buffer containing magnesium (1x TAE ( $\mathrm{pH} 8), 10-20 \mathrm{mM} \mathrm{MgCl}_{2}$, depending on the nanostructure) and buffers containing different concentrations of spermidine trihydrochloride $\left(\mathrm{C}_{7} \mathrm{H}_{22} \mathrm{~N}_{3} \mathrm{Cl}_{3} / \mathrm{Spd} \cdot 3 \mathrm{HCl}\right)$ (Sigma). DNA staple strands were purchased from Eurofins MWG, M13 scaffold variants (p7560, p7249) were kindly provided by F. Praetorius (Dietz lab, TUM) ${ }^{4}$. The sample solutions were subjected to a thermal annealing ramp that cooled from $65^{\circ} \mathrm{C}$ to $20^{\circ} \mathrm{C}$ over a time course of several hours (different annealing times were used depending on the target nanostructure). All chemicals were purchased from Carl Roth, Germany unless otherwise specified. Cadnano designs and nucleotide sequences of the different structures are provided below (Figure S1, Supporting tables S1-S3).

Agarose gel electrophoresis (AGE). All reaction products after folding and exposure to electric fields were analyzed in $2 \%$ agarose gels containing $1 \times \mathrm{TAE}, 12.5 \mathrm{mM} \mathrm{MgCl}$, at $100 \mathrm{~V}$ in a gel box immersed in an ice water bath for 2.5 hours. The gels were post-stained with SYBR gold (Invitrogen, USA) for 40 minutes and destained with buffer. The gels were scanned on a Typhoon FLV 9000 laser scanner and analyzed with the software package Fiji ${ }^{5}$. 
Transmission electron microscopy (TEM). Purified DNA nanostructures were adsorbed on glow-discharged formvar-supported carbon-coated Cu400 TEM grids (Science Services, Munich, Germany) and stained using a $2 \%$ aqueous uranyl formate solution containing $25 \mathrm{mM}$ sodium hydroxide. Imaging was performed using a Philips CM100 electron microscope operated at $100 \mathrm{kV}$. Images were acquired at 28500× magnification using an AMT 4 Megapixel CCD camera. Class averaging of DNA nanostructures was performed by using the xmipp protocol in the software package Scipion ${ }^{6}$.

Modification and purification of DNA origami nanostructures. DNA origami nanostructures (42HB) were fluorescently labeled at 21 positions via hybridization to Atto647N-modified oligonucleotides to facilitate detection by confocal microscopy. To this end, the corresponding DNA staple strands were extended at the 3' end with a 22 nt long sequence complementary to the 5'-labelled DNA strand. The sequence of the extension and dye labelled DNA strand are provided in table 1. Mg-nanostructures were purified using PEG purification ${ }^{7}$ and Spdnanostructures were purified by size exclusion chromatography (SEC) using sephacryl resins (SR 300) (GE Healthcare Life Sciences) to remove excess DNA staples ${ }^{8}$. Sephacryl resins were equilibrated with MillliQ water and $50 \% \mathrm{v} / \mathrm{v}$ slurry was prepared. The resins were packed into Pierce centrifugal columns (Invitrogen) by spinning the columns at $800 \mathrm{rcf}$ for 3 minutes at $15^{\circ} \mathrm{C}$. A volume of $100 \mu 1$ unpurified Spd-nanostructures was subsequently passed through two spin columns packed with different resin volumes (400 and $260 \mu 1$ respectively) and spun at 800 rcf for 3 minutes at $15^{\circ} \mathrm{C}$.

We found that established methods of DNA origami purification such as PEG precipitation ${ }^{7}$, spin column filtration and gel extraction ${ }^{8,9}$ were not well suited for Spd-nanostructures. High sodium chloride concentrations used in PEG precipitation unfolded the Spd-nanostructures, potentially due to the competition between the monovalent and trivalent ions ${ }^{10}$. Spdnanostructures interacted with and clogged spin column filter membranes, making the filters 
unsuitable for purification. Gel extraction resulted in contamination of the structures with ions contained in the gel buffer (data not shown). We therefore used a protocol based on size exclusion chromatography (SEC) that left the Spd-nanostructures intact without changing the composition of the charge-compensating counterions.

As an alternative strategy to stabilize Mg-folded origami structures in spermidine buffer postfolding, we also found that Mg-folded structures can be first PEG purified, followed by a buffer exchange to an Spd-buffer (data not shown).

DNA nanostructures in electric fields. DNA nanostructures folded in $\mathrm{Mg}^{2+}$ or $\mathrm{Spd}^{3+}$-containing buffers as described above and diluted to a concentration of $10 \mathrm{nM}$ in the cell culture medium RPMI (Gibco, USA, conductivity $\approx 1.5 \mathrm{~S} / \mathrm{m}$ ) were subjected to different electric field pulses using a BTX electroporator ECM 399. The strength and duration of the pulses was varied by altering the length of the cuvettes, the voltage applied and the capacitance mode $(\mathrm{C}=1050 \mu \mathrm{F}$ or $36 \mu \mathrm{F}$ ) selected on the instrument. Electroporation cuvettes with $0.1 \mathrm{~cm}$ electrode distance (electrode area $0.5 \mathrm{~cm} \times 1.1 \mathrm{~cm}$ ) were filled with a sample volume of $100 \mu 1$, while cuvettes with $0.4 \mathrm{~cm}$ distance (electrode area $2 \times 1.1 \mathrm{~cm}$ ) were filled with $800 \mu 1$. Electric field pulse and electroporation experiments were performed within 5 minutes after dilution in order to avoid disintegration of the structures in the RPMI medium (with $\left[\mathrm{Mg}^{2+}\right] \approx 0.4 \mathrm{mM} \mathrm{RPMI}$ is essentially Mg-free) ${ }^{11}$.

Electrotransfection of Spd-nanostructures in Jurkat cells. One day before electroporation, a suspension culture of Jurkat cells was split in tissue culture flasks (T-25, VWR) in growth medium (RPMI (Gibco, USA)) supplemented with 10\% fetal bovine serum, $1 \%$ glutamax-I, and $1 \%$ penicillin and streptomycin. Cells were seeded in each flask at a density of $3 \times 10^{6} / \mathrm{ml}$ (counted by a hemocytometer) and incubated overnight at $37^{\circ} \mathrm{C}$ in a humidified atmosphere containing $5 \% \mathrm{CO}_{2}$. On the following day, cells were washed with PBS (Phosphate buffered saline) and resuspended in $650 \mu \mathrm{l}$ RPMI (Gibco, USA)) not supplemented with additional 
components and added to $0.4 \mathrm{~cm}$ electroporation cuvettes. A final concentration of $10 \mathrm{nM}$ purified Atto647N labelled DNA nanostructures (Spd/Mg-nanostructures) was added to cells. The cuvettes were kept on ice all the time. Electroporation was carried out at $200 \mathrm{~V}$ (low voltage mode, $\mathrm{C}=1050 \mu \mathrm{F})$ using a BTX electroporator ECM 399. The electroporated cells were added to fully supplemented growth media as above and kept in an incubator at $37^{\circ} \mathrm{C}$ in a humidified atmosphere containing 5\% $\mathrm{CO}_{2}$ for 3-4 hours to allow the cells to recover before imaging.

Confocal Fluorescence Microscopy. Before imaging, growth medium was removed and cells were washed with PBS. Benzonase nuclease (EMD Millipore) treatment prior to imaging was carried out by incubating the cells for 30 minutes in a solution of PBS containing at least 0.5 $\mathrm{U} / \mu 1$ of nuclease to the cells to dispense of DNA nanostructures outside of the cells. Cell organelles were stained in order to assess the intracellular localization of the DNA nanostructures introduced. LysoSensor Green (DND-189, Invitrogen, USA) and CellMask plasma membrane stain orange (Invitrogen, USA) were used to stain the lysosomes and cell membrane respectively according to the manufacturer's protocol. Jurkat cells were washed and supplied with fully supplemented growth medium and seeded in collagen coated IBIDI ${ }^{\mathrm{TM}}$ chambers to facilitate their adherence. Live cells were imaged using a Leica SP II inverted fluorescence confocal microscope (Leica) in a sequential scan mode and analyzed using Fiji. Fluorescence filters used were $488 \mathrm{~nm} \mathrm{Ex/} \mathrm{510-540} \mathrm{nm} \mathrm{Em} \mathrm{for} \mathrm{Lysosensor} \mathrm{Green,} 561 \mathrm{~nm}$ Ex/ 565-600 nm Em and $633 \mathrm{~nm} \mathrm{Ex/660-700} \mathrm{nm} \mathrm{for} \mathrm{Atto647N-DNA} \mathrm{nanostructures.}$

Cell viability assays. The viability of Jurkat cells after being subjected to electroporation of DNA origami nanostructures was evaluated by two different assays, namely CyQuant Direct assay (Thermo Scientific) and PrestoBlue cell viability assay (Thermo Scientific). Both these assays determined the cell viability by orthogonal mechanisms.

The CyQUANT Direct assay is based on a live cell-permeant DNA-binding dye (Ex=480 nm, $\mathrm{Em}=530 \mathrm{~nm}$ ) in combination with an impermeable suppression reagent. The suppression agent 
blocks staining of dead cells and cells with compromised cell membranes, causing only staining of healthy cells with intact membranes. The increase in fluorescence of DNA-binding dye is proportional to the increasing cell number over time, thus confirming cell proliferation and growth. Jurkat cells $\left(10^{5}\right.$ cells $\left./ \mathrm{ml}\right)$ subjected to different conditions- no electroporation, electroporation without any DNA origami nanostructures, electroporation with 42HB (cf. Supporting Information section "Transfection of Spd-nanostructures in Jurkat cells via electroporation"), were seeded in triplicates (n=3) by adding $100 \mu$ of cell suspension into the wells of a 96 -well plate for each time point $(0,24,48$, or 72 hours after seeding). At each time point, cell proliferation was measured by CyQUANT Direct assay following the manufacturer's protocol. Fluorescence was measured using a FLUOstar Omega plate reader at $530 \mathrm{~nm}$. The fluorescence observed at different time points was normalized to fluorescence readings at $\mathrm{t}=0$ hours. It was observed that the fluorescence increased with time suggesting an increase in cell number, thereby confirming cell proliferation and growth (Figure S15 A).

The PrestoBlue assay uses a cell permeable non fluorescent, blue colored resazurin-based reagent $(\mathrm{Ex}=569 \mathrm{~nm} / \mathrm{Em}=586 \mathrm{~nm})$ which functions as a cell viability indicator. The PrestoBlue reagent gets modified to the red colored highly fluorescent compound resorufin by the reducing environment of the viable cells. The increase in fluorescence is in proportional to the increasing cell number over time, thus corroborating cell viability. Jurkat cells $\left(10^{6}\right.$ cells $\left./ \mathrm{ml}\right)$ subjected to different conditions as mentioned above were seeded in triplicates $(n=3)$ by adding $90 \mu$ of cell suspension into the wells of a 96-well plate for each time point $(0,3,6$, or 24 hours after seeding). Cell viability was measured using PrestoBlue assay at each time point following the manufacturer's protocol. Fluorescence was measured on a FLUOstar Omega plate reader at 590 $\mathrm{nm}$. The fluorescence observed at different time points was normalized to fluorescence readings at 0 hour. It was observed that the fluorescence increased with time suggesting an increase in cell number, thereby confirming cell viability (Figure S15 B). 
Stability in cell lysate. Cell lysate buffer (10X) was purchased from CST. Jurkat cell lysate $\left(10^{6}\right.$ cells/ml) was prepared as per manufacturer's protocol. Cell lysates corresponding to different cell numbers were obtained by dilution of lysate. The quality of cell lysate was checked using BCA assay and the protein content varied linearly with the corresponding cell number. $20 \mathrm{nM}$ $42 \mathrm{HB}$ was incubated with lysate corresponding to different cell number for different times ( 0 and 6 hours) at $37^{\circ} \mathrm{C}$. The survival of $42 \mathrm{HB}$ was then analysed by TEM and agarose gels (Figure S16). 


\section{Supporting figures}

A

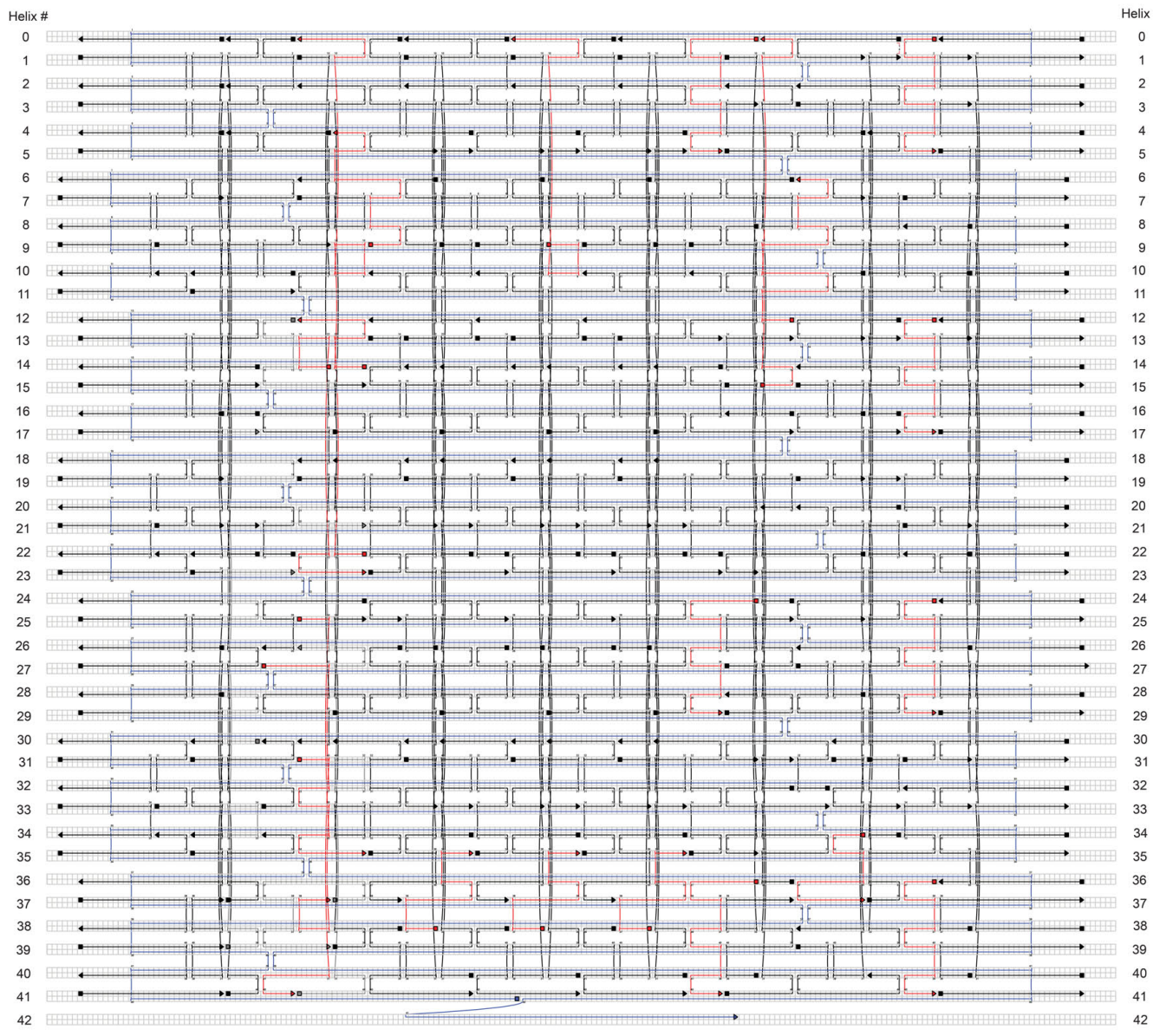

B

$5^{4} 3^{2}-1$

$67^{8}, 9^{10} 11$

(17) 16$\left.)^{14}\right)_{13}^{12}$

$\left.18)^{20} 21\right)^{22}(23$

(29) 28$\left.)^{27}\right)^{26}(2)^{24}$

(30) $\left.312^{32}\right)_{33}^{34}$

(41 $\left.\left.{ }^{40} 39\right)^{38} 37\right)^{36}$

$+\square$

?

$\square$

(2)

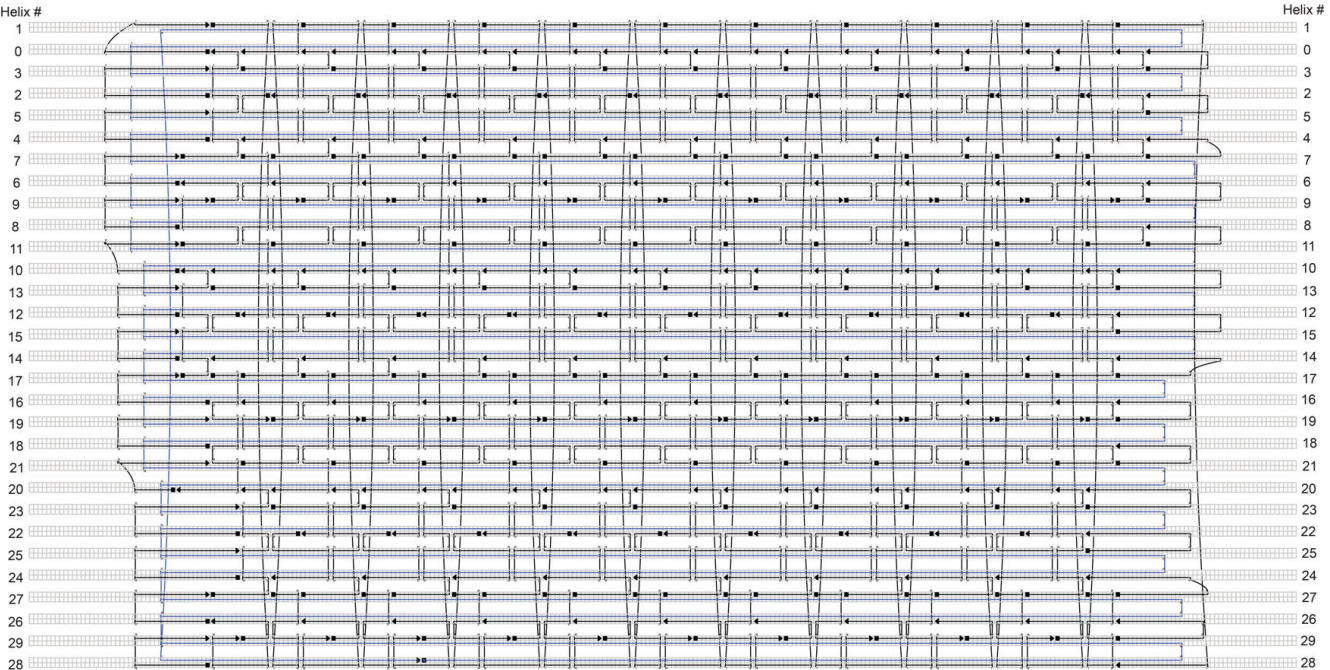


C

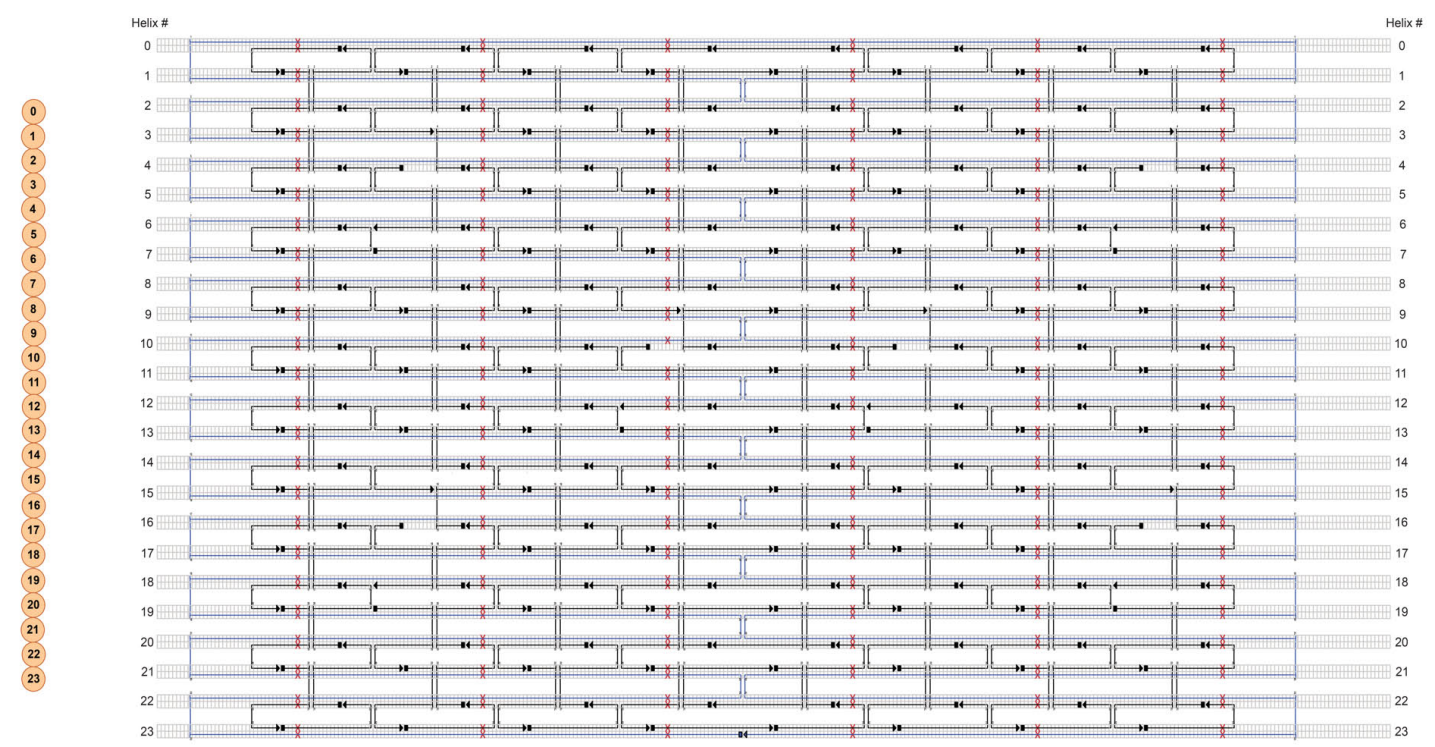

Figure S1. Cadnano diagrams of the three DNA-origami nanostructures used in this work. Scaffold strands are depicted in blue and staple strands in black. (A) 42HB, staple strands which are extended for hybridization with dye labelled complementary DNA strand are depicted in red, (B) Tube, (C) tCRRO. 
A

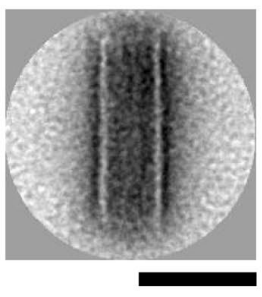

B

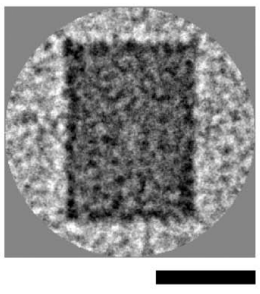

$\begin{array}{lllllllllllllllllllll}\mathrm{L} & \mathrm{S} & \mathrm{C} & 1 & 2 & 3 & 4 & 5 & 6 & 7 & 8 & 9 & 10 & 11 & 12 & 13 & 14 & \mathrm{C} & \mathrm{S} & \mathrm{L}\end{array}$
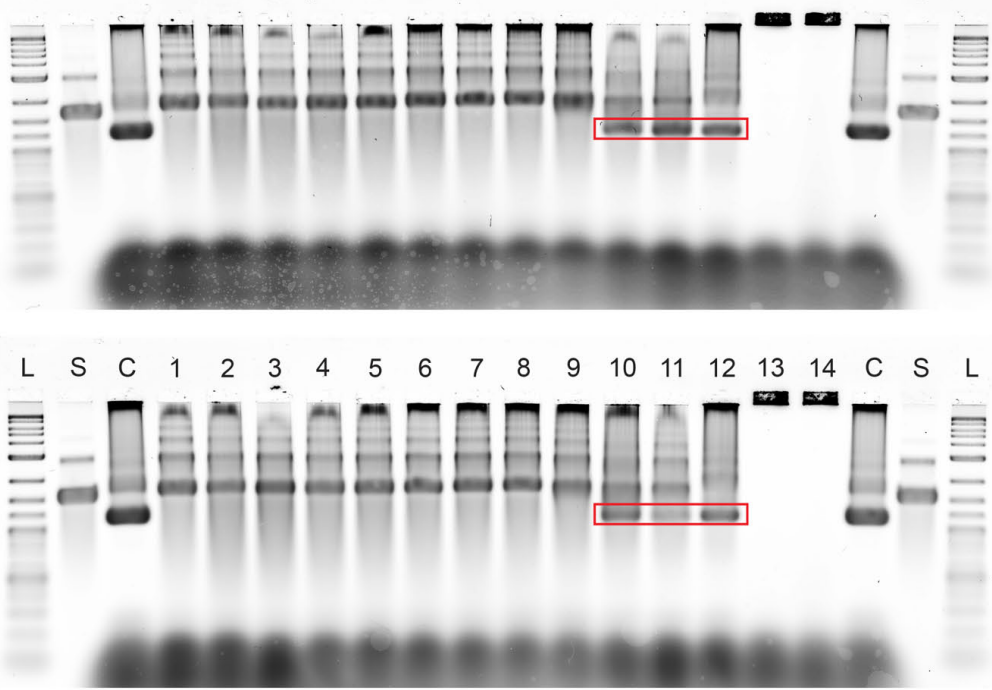

Figure S2. Class averaged TEM images of DNA origami nanostructures folded in $\mathrm{Spd}^{3+}$ and agarose gel electrophoresis for screening of $\mathrm{Spd}^{3+}$ concentrations required for folding 50nM DNA origami nanostructures. (A) Tubes, (B) tCRRO. L: 2-Log Ladder, C: DNA origami nanostructures folded in traditional buffer containing magnesium (control). $\mathrm{Spd}^{3+}$ concentrations in the numbered lanes are: 1: $0 \mu \mathrm{M}, 2: 50 \mu \mathrm{M}, 3: 100 \mu \mathrm{M}, 4: 200 \mu \mathrm{M}, 5: 300 \mu \mathrm{M}, 6: 400 \mu \mathrm{M}, 7$ : $500 \mu \mathrm{M}, 8: 600 \mu \mathrm{M}, 9: 700 \mu \mathrm{M}, 10: 800 \mu \mathrm{M}, 11: 900 \mu \mathrm{M}, 12: 1 \mathrm{mM}, 13: 5 \mathrm{mM}, 14: 10 \mathrm{mM}$. Red boxes highlight bands corresponding to the correctly folded DNA origami nanostructures. Scale bar $=50 \mathrm{~nm}$.

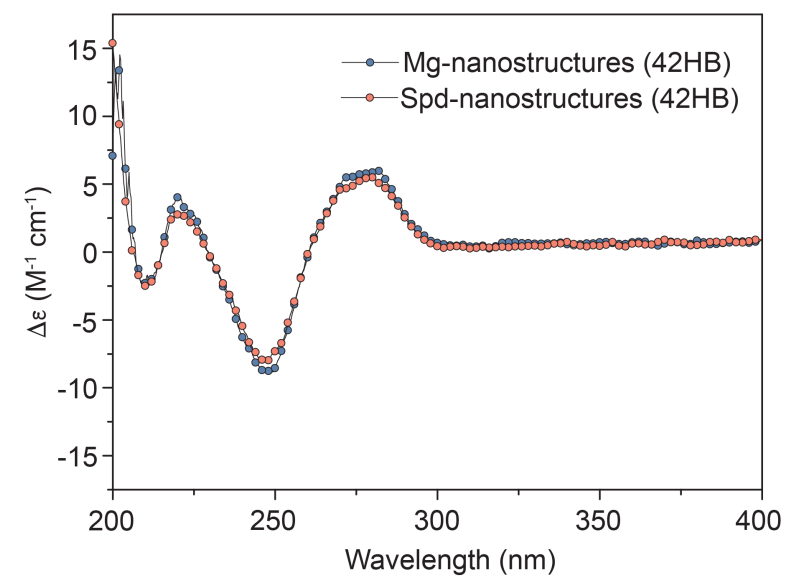

Figure S3. Circular dichroism spectra of $42 \mathrm{HB}$ folded in traditional buffer containing magnesium (blue $\ldots$ symbol curve) and in $\operatorname{Spd}^{3+}(\multimap$ pink curve). The spectra were recorded from $200 \mathrm{~nm}$ to $400 \mathrm{~nm}$ with data being collected every $0.2 \mathrm{~nm}$ on a Jasco J-815 CD spectrometer. For the curves, the data was taken every $0.2 \mathrm{~nm}$, symbols are shown only every 10 nm. 


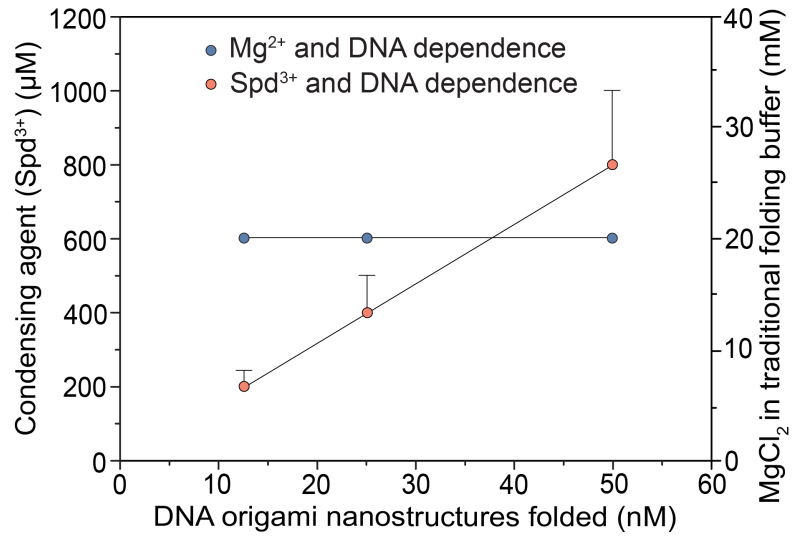

Figure S4. Relation between the concentration of folding buffer and concentration of DNA origami nanostructures to be folded. The concentration of $\mathrm{Spd}^{3+}$ required for folding is roughly proportional to the concentration of DNA nanostructures to be folded (pink circle symbols (०)). At a particular DNA concentration, folding occurs over a narrow range of $\mathrm{Spd}^{3+}$ as depicted by in the above figure. Blue circle symbols $(\bullet)$ show that folding of DNA origami nanostructures is independent of the concentration of DNA and folding occurs at the same concentration of $\mathrm{MgCl}_{2}$ in traditional buffer $($ at $\approx 20 \mathrm{mM})$.

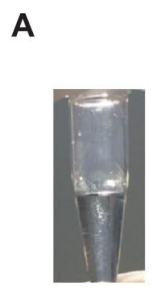

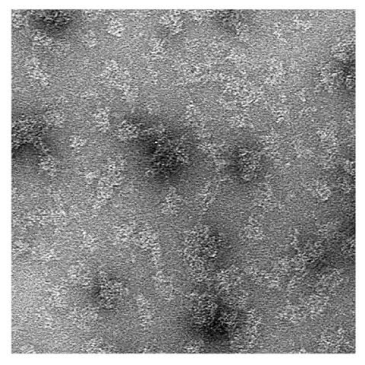

$200 \mu \mathrm{M}$ (No folding)
B

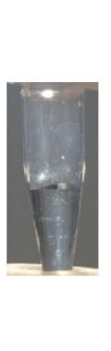

$800 \mu \mathrm{M}$ (folding)

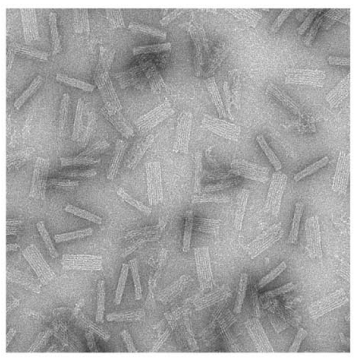

C

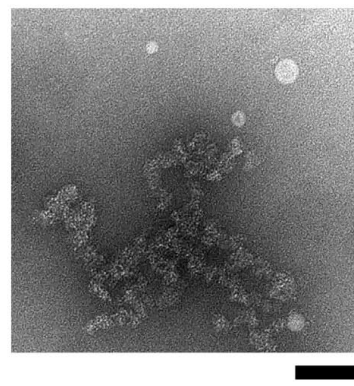

10mM (Aggregation)

Figure S5. Folding reactions and corresponding TEM micrographs for $42 \mathrm{HB}$ at different concentrations of $\mathrm{Spd}^{3+}$. Concentration of $42 \mathrm{HB}$ to be folded is $50 \mathrm{nM}$. Aggregation and precipitation of DNA scaffold and staples at higher concentrations of $\mathrm{Spd}^{3+}$ is observed. (A) 200 $\mu \mathrm{M}$ (No folding), (B) $800 \mu \mathrm{M}$ (folding concentration for $50 \mathrm{nM} 42 \mathrm{HB}$ ), (C) $10 \mathrm{mM}$ (aggregation of DNA before thermal annealing cycle). Scale bar $=100 \mathrm{~nm}$. 
A

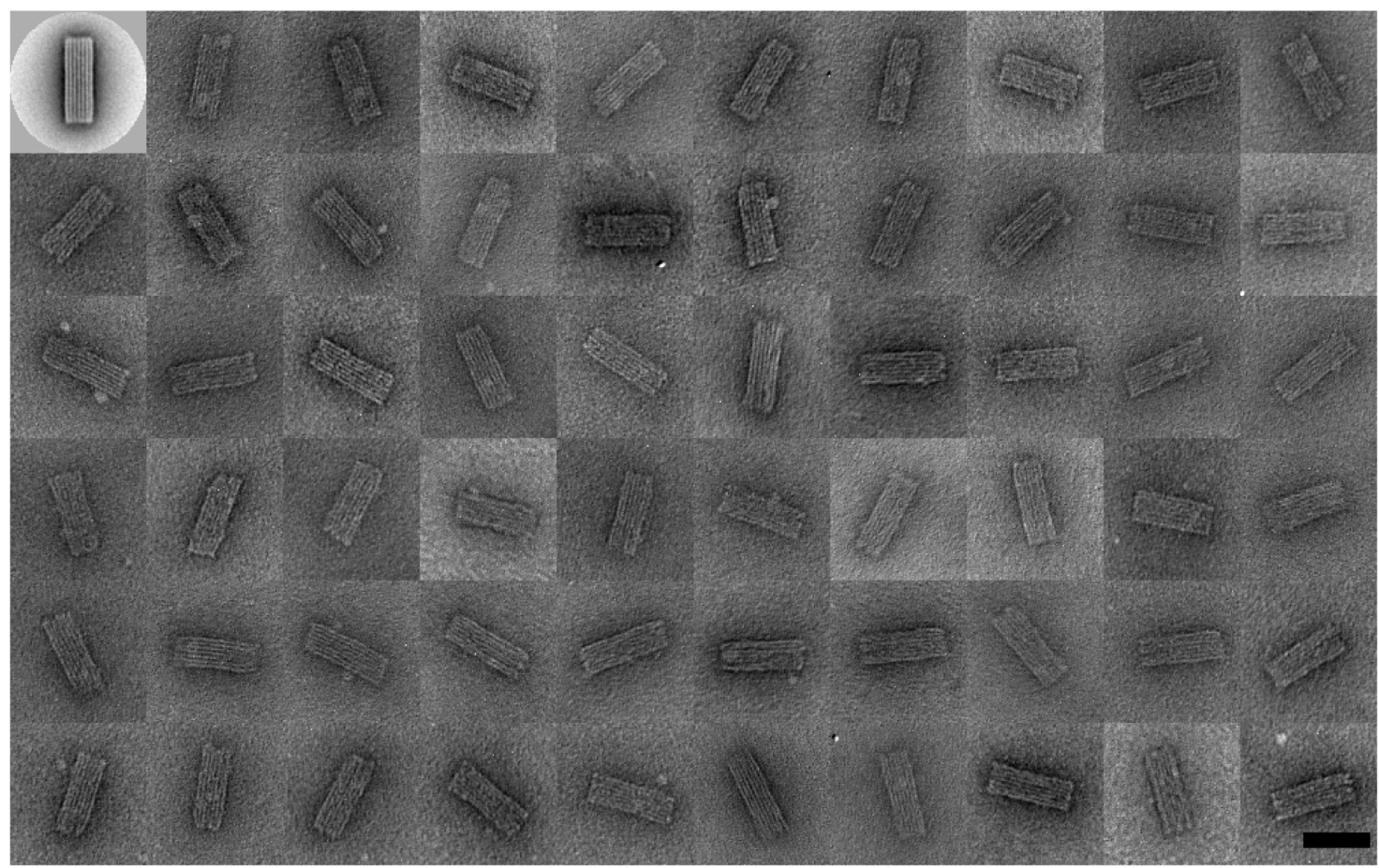

B

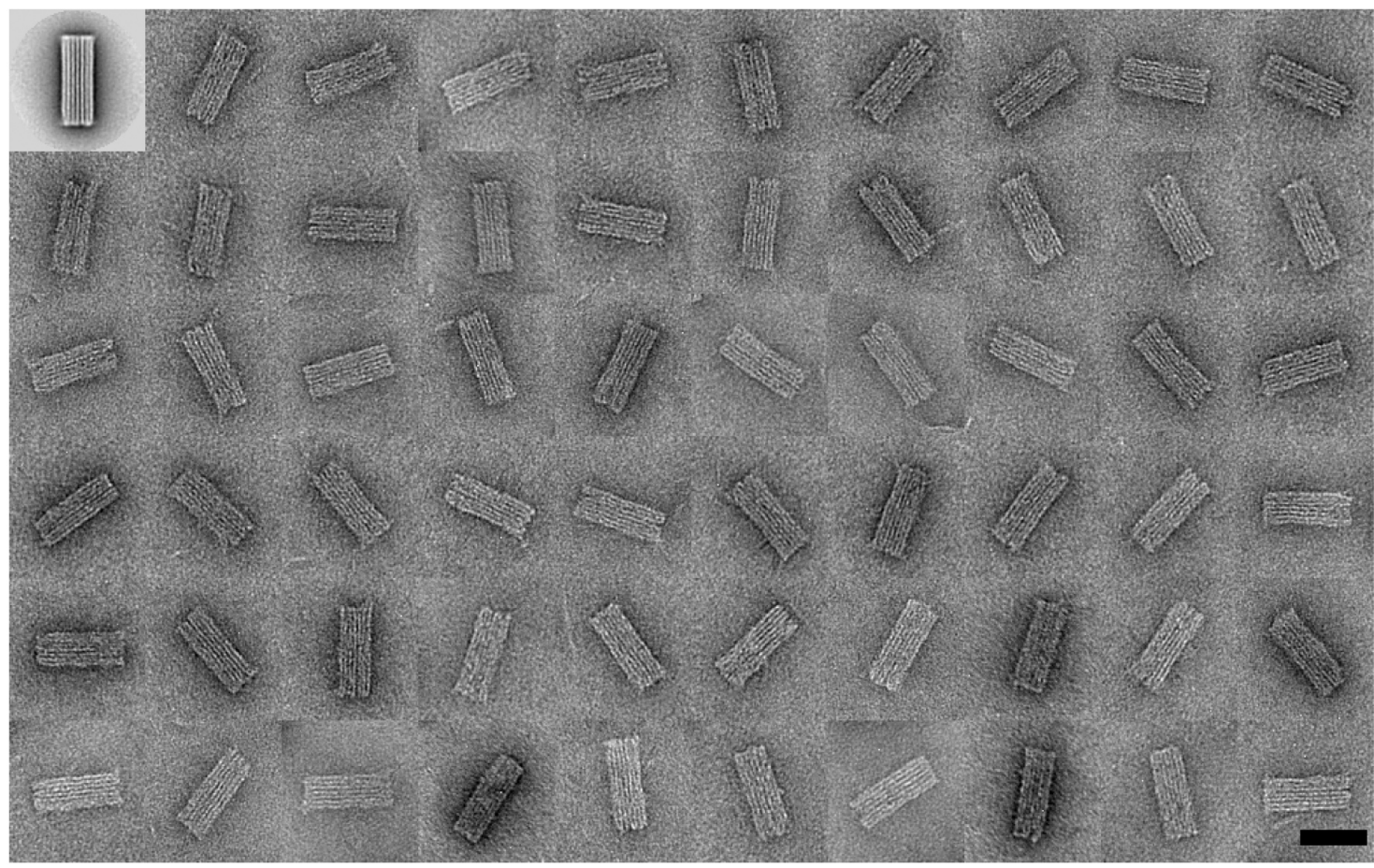

Figure S6. Negative-stain TEM micrographs of $42 \mathrm{HB}$ folded in traditional buffer containing magnesium (A) and $\mathrm{Spd}^{3+}$ (B) respectively. Each micrograph: $100.5 \mathrm{~nm}$ x $100.5 \mathrm{~nm}$. Top row, image $\# 1$ = class average micrograph. Scale bar: $50 \mathrm{~nm}$. The dimensions of $42 \mathrm{HB}$ (length $=60$ $\mathrm{nm}$, width $=26 \mathrm{~nm}$, height $\approx 16 \mathrm{~nm}$ ) were found to be the same when folded in either magnesium containing buffer or $\mathrm{Spd}^{3+}$. 
A

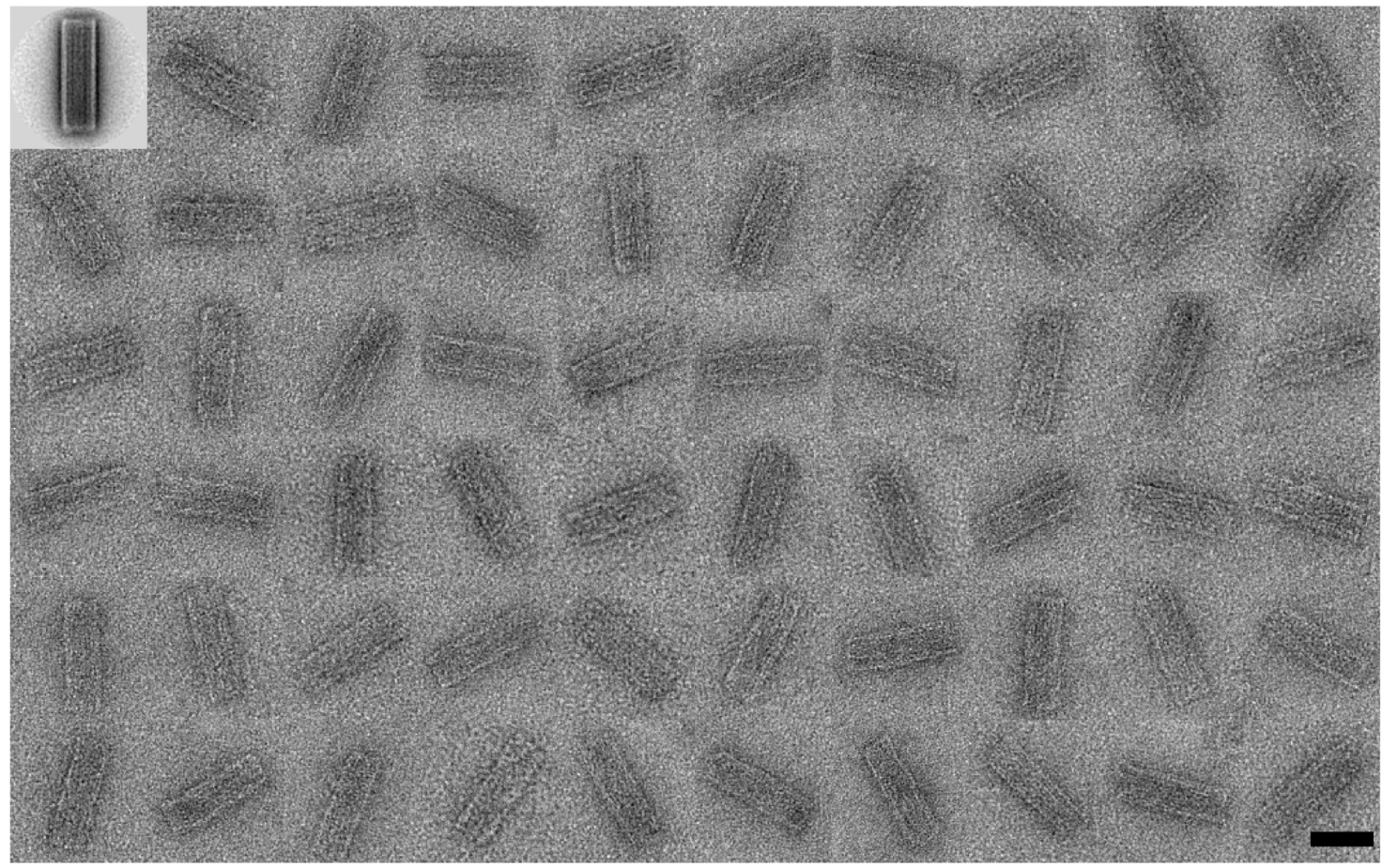

B

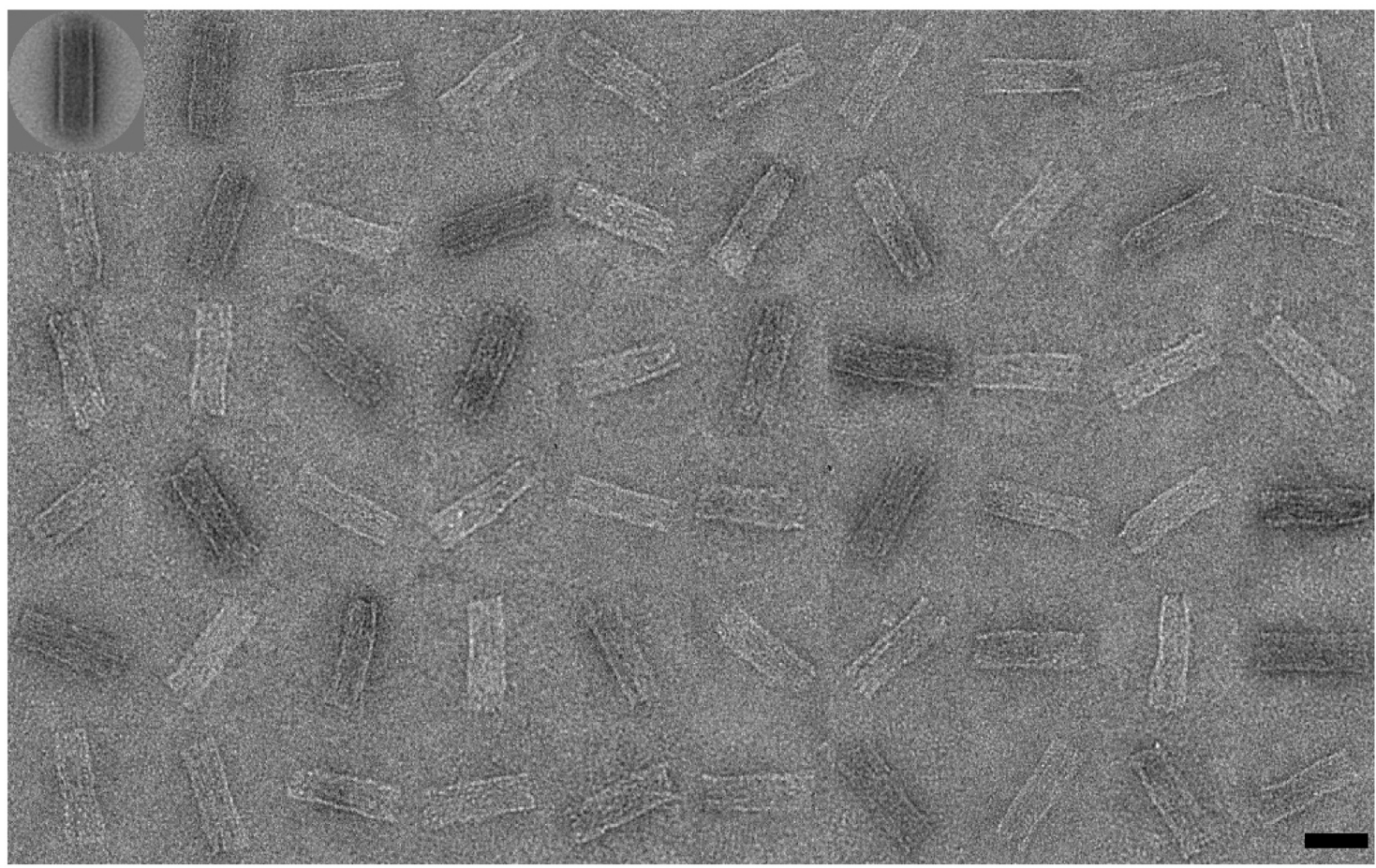

Figure S7. Negative-stain TEM micrographs of tube folded in traditional buffer containing magnesium (A) and $\mathrm{Spd}^{3+}(\mathrm{B})$ respectively. Each micrograph: $106.6 \mathrm{~nm}$ x $106.6 \mathrm{~nm}$. Top row, image $\# 1$ = class average micrograph. Scale bar: $50 \mathrm{~nm}$. The dimensions of tube (length = 83 $\mathrm{nm}$, diameter $1 \approx 23 \mathrm{~nm}$, diameter $2 \approx 29 \mathrm{~nm}$ ) were found to be the same when folded in either magnesium containing buffer or $\operatorname{Spd}^{3+}$. 
A

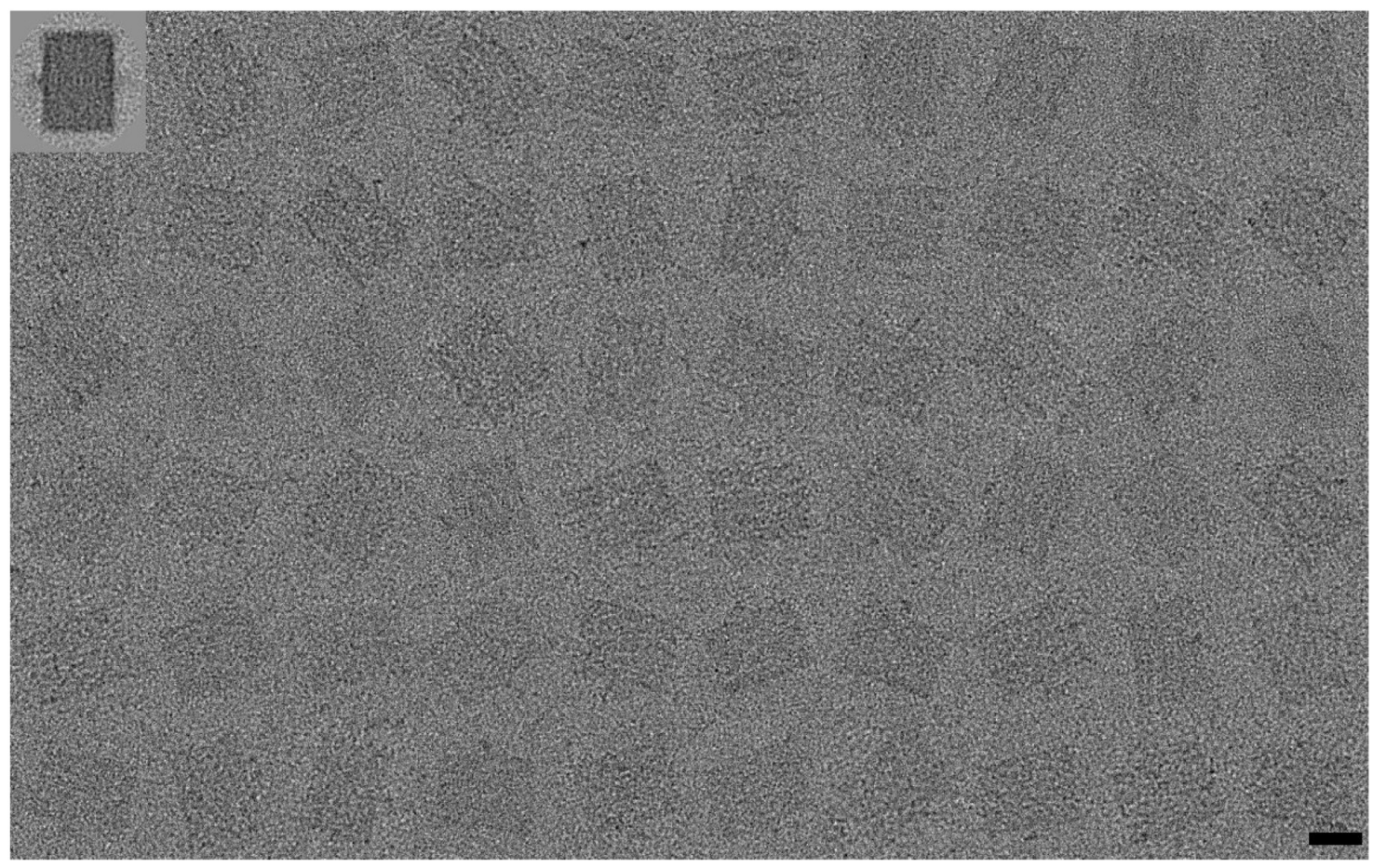

B

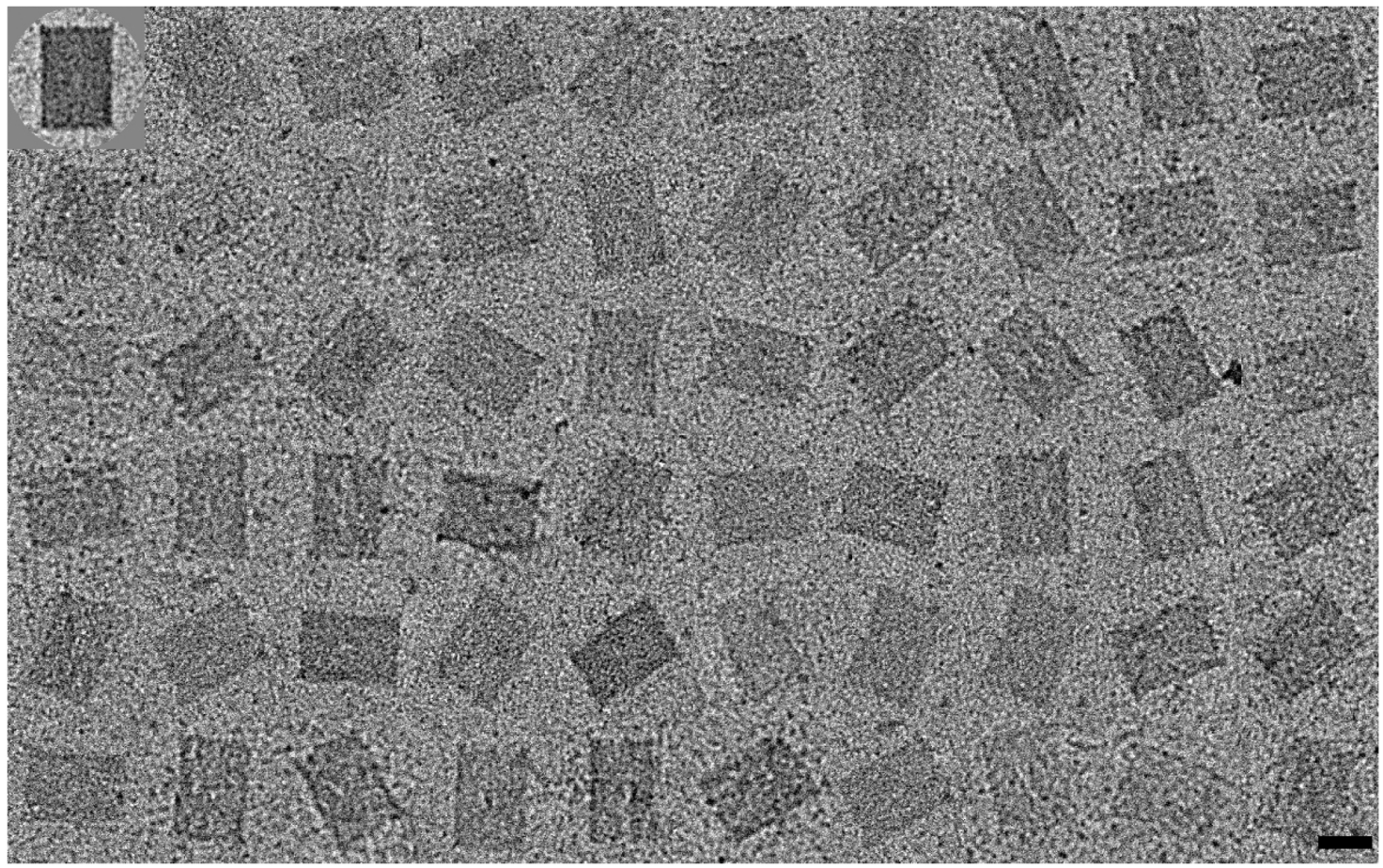

Figure S8. Positive-stain TEM micrographs of tCRRO folded in traditional buffer containing magnesium (A) and $\mathrm{Spd}^{3+}$ (B) respectively. Each micrograph: $125.7 \mathrm{~nm}$ x $125.7 \mathrm{~nm}$. Top row, image $\# 1$ = class average micrograph. Scale bar: $50 \mathrm{~nm}$. The dimensions of tube (length $=90$ $\mathrm{nm}$, width $=65 \mathrm{~nm}$, height $=2 \mathrm{~nm}$ ) were found to be the same when folded in either magnesium containing buffer or $\mathrm{Spd}^{3+}$. 
A

\begin{tabular}{|c|c|c|c|c|c|c|}
\hline & \multicolumn{5}{|c|}{ Low voltage $(\mathrm{LV})$ mode, $\mathrm{C}=1050 \mu \mathrm{F}$} \\
\hline Voltage (V) & OV & $10 \mathrm{~V}$ & $50 \mathrm{~V}$ & $100 \mathrm{~V}$ & $200 \mathrm{~V}$ & $400 \mathrm{~V}$ \\
\hline \begin{tabular}{|c|} 
Path length, \\
$(\mathrm{cm})$
\end{tabular} & - & 0.1 & 0.1 & 0.1 & 0.1 & 0.1 \\
\hline $\begin{array}{c}\text { Electric field } \\
(\overrightarrow{\mathrm{E}}),(\mathrm{V} / \mathrm{cm})\end{array}$ & - & 100 & 500 & 1000 & 2000 & 4000 \\
\hline $\begin{array}{l}\text { Path length, } \\
(\mathrm{cm})\end{array}$ & - & 0.4 & 0.4 & 0.4 & 0.4 & 0.4 \\
\hline $\begin{array}{c}\text { Electric field } \\
(\vec{E}),(V / c m)\end{array}$ & - & 25 & 125 & 250 & 500 & 1000 \\
\hline
\end{tabular}

B

Low voltage (LV) mode, $\mathrm{C}=1050 \mu \mathrm{F}$

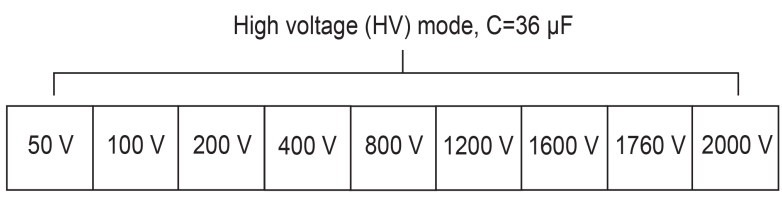

\begin{tabular}{|c|c|c|c|c|c|c|c|c|}
\hline 0.1 & 0.1 & 0.1 & 0.1 & 0.1 & 0.1 & 0.1 & 0.1 & 0.1 \\
\hline 500 & 1000 & 2000 & 4000 & 8000 & 12000 & 16000 & 17600 & 20000 \\
\hline
\end{tabular}

\begin{tabular}{|c|c|c|c|c|c|c|c|c|}
\hline 0.4 & 0.4 & 0.4 & 0.4 & 0.4 & 0.4 & 0.4 & 0.4 & 0.4 \\
\hline 125 & 250 & 500 & 1000 & 2000 & 3000 & 4000 & 4400 & 5000 \\
\hline
\end{tabular}

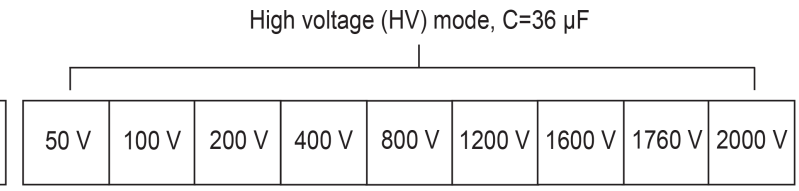

\begin{tabular}{|c|c|c|c|c|c|c|}
\hline $\begin{array}{c}\text { Path length, } \\
(\mathrm{cm})\end{array}$ & - & 0.1 & 0.1 & 0.1 & 0.1 & 0.1 \\
\hline $\begin{array}{r}\text { Electric field } \\
(\overrightarrow{\mathrm{E}}),(\mathrm{V} / \mathrm{cm})\end{array}$ & - & 100 & 500 & 1000 & 2000 & 4000 \\
\hline
\end{tabular}

\begin{tabular}{|c|c|c|c|c|c|c|c|c|}
\hline 0.1 & 0.1 & 0.1 & 0.1 & 0.1 & 0.1 & 0.1 & 0.1 & 0.1 \\
\hline 500 & 1000 & 2000 & 4000 & 8000 & 12000 & 16000 & 17600 & 20000 \\
\hline
\end{tabular}

\begin{tabular}{|c|c|c|c|c|c|c|}
\hline $\begin{array}{l}\text { Path length, } \\
(\mathrm{cm})\end{array}$ & - & 0.4 & 0.4 & 0.4 & 0.4 & 0.4 \\
\hline $\begin{array}{c}\text { Electric field } \\
(\overrightarrow{\mathrm{E}}),(\mathrm{V} / \mathrm{cm})\end{array}$ & - & 25 & 125 & 250 & 500 & 1000 \\
\hline
\end{tabular}

DNA nanostructures do not survive

\begin{tabular}{|c|c|c|c|c|c|c|c|c|}
\hline 0.4 & 0.4 & 0.4 & 0.4 & 0.4 & 0.4 & 0.4 & 0.4 & 0.4 \\
\hline 125 & 250 & 500 & 1000 & 2000 & 3000 & 4000 & 4400 & 5000 \\
\hline
\end{tabular}

DNA nanostructures survive
Conditions favorable for mammalian cell transfection

Figure S9. Effect of different electric field strengths on the stability of $42 \mathrm{HB}$ folded in different buffers. (A) Mg-nanostructures. (B) Spd-nanostructures. Mg- or Spd- nanostructures diluted in RPMI and subjected to a range of electric field strengths both in LV and HV mode. The strength of the field is varied by using cuvettes of different path lengths, 0.1 and $0.4 \mathrm{~cm}$. Voltages, path length and the corresponding electric field strengths analogous to the modes are specified in rows \#1; \#2, \#4; \#3, \#5 respectively, in $\mathrm{A}$ and $\mathrm{B}$. The rows equivalent to electric field strengths (\#3, \#5) are color coded with respect to survival of nanostructures (pink/green) and conditions favorable for mammalian cell transfection (purple). Mg- structures sustained the long ( $\tau=12 \mathrm{~ms}$ ) electric fields pulses only below $\mathrm{E} \approx 125 \mathrm{~V} / \mathrm{cm}$, and the short duration $(\tau=0.4 \mathrm{~ms})$ pulses below $\mathrm{E} \approx 4000 \mathrm{~V} / \mathrm{cm}$. The Mg-structures failed to survive at conditions favorable for mammalian cell transfection. On the contrary, Spd-nanostructures survived all conditions. However, at electric field strengths of $E \approx 17600 \mathrm{~V} / \mathrm{cm}$ and higher their stability is compromised (cf. Figure S11 A). 
A

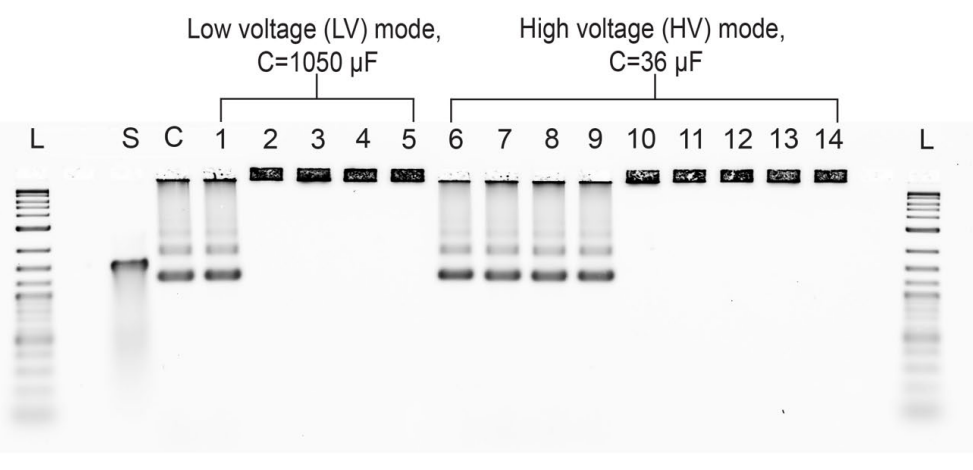

B

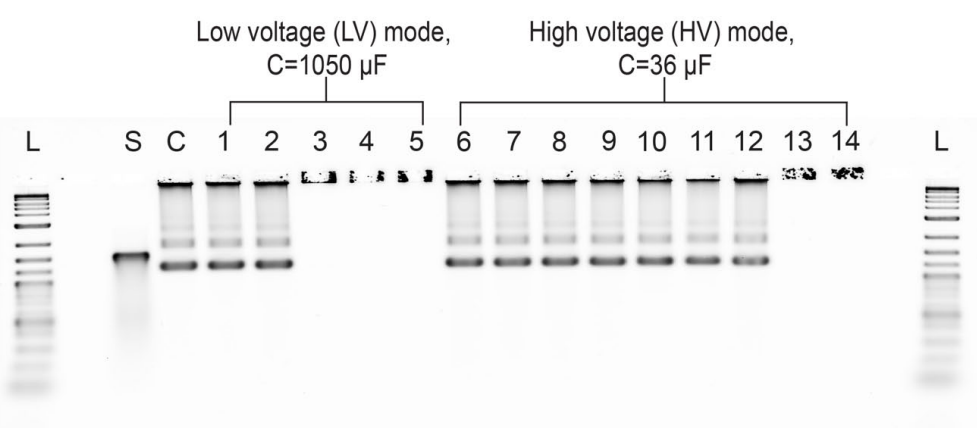

Figure S10. Agarose gel electrophoresis showing the stability of Mg-nanostructures (42HB) diluted in RPMI and subjected to a range of electric field strengths both in LV and HV mode. L: 2-Log Ladder, C: Control (No electric field). (A) $0.1 \mathrm{~cm}$ path length. Electric fields corresponding to the numbered lanes are: 1: $100 \mathrm{~V} / \mathrm{cm}, 2: 500 \mathrm{~V} / \mathrm{cm}, 3: 1000 \mathrm{~V} / \mathrm{cm}, 4: 2000$ $\mathrm{V} / \mathrm{cm}, 5: 4000 \mathrm{~V} / \mathrm{cm}$ in the low voltage mode ( $\mathrm{LV}$ mode, $1050 \mu \mathrm{F}$ ) and 6: $500 \mathrm{~V} / \mathrm{cm}, 7: 1000$ $\mathrm{V} / \mathrm{cm}$, 8: $2000 \mathrm{~V} / \mathrm{cm}$, 9: $4000 \mathrm{~V} / \mathrm{cm}, 10: 8000 \mathrm{~V} / \mathrm{cm}, 11: 12000 \mathrm{~V} / \mathrm{cm}, 12: 16000 \mathrm{~V} / \mathrm{cm}, 13$ : $17600 \mathrm{~V} / \mathrm{cm}, 14: 20000 \mathrm{~V} / \mathrm{cm}$ in the high voltage mode (HV mode, $36 \mu \mathrm{F}$ ). (B) $0.4 \mathrm{~cm}$ path length. Electric fields corresponding to the numbered lanes are: 1: $25 \mathrm{~V} / \mathrm{cm}, 2: 125 \mathrm{~V} / \mathrm{cm}, 3: 250$ $\mathrm{V} / \mathrm{cm}$, 4: $500 \mathrm{~V} / \mathrm{cm}, 5: 1000 \mathrm{~V} / \mathrm{cm}$ in the low voltage mode (LV mode, $1050 \mu \mathrm{F}$ ) and 6: 125 $\mathrm{V} / \mathrm{cm}, 7: 250 \mathrm{~V} / \mathrm{cm}, 8: 500 \mathrm{~V} / \mathrm{cm}, 9: 1000 \mathrm{~V} / \mathrm{cm}, 10: 2000 \mathrm{~V} / \mathrm{cm}, 11: 3000 \mathrm{~V} / \mathrm{cm}, 12: 4000 \mathrm{~V} / \mathrm{cm}$, 13: $4400 \mathrm{~V} / \mathrm{cm}, 14: 5000 \mathrm{~V} / \mathrm{cm}$ in the high voltage mode (HV mode, $36 \mu \mathrm{F}$ ). These observations are also mentioned in a tabulated form in figure S9 A. 


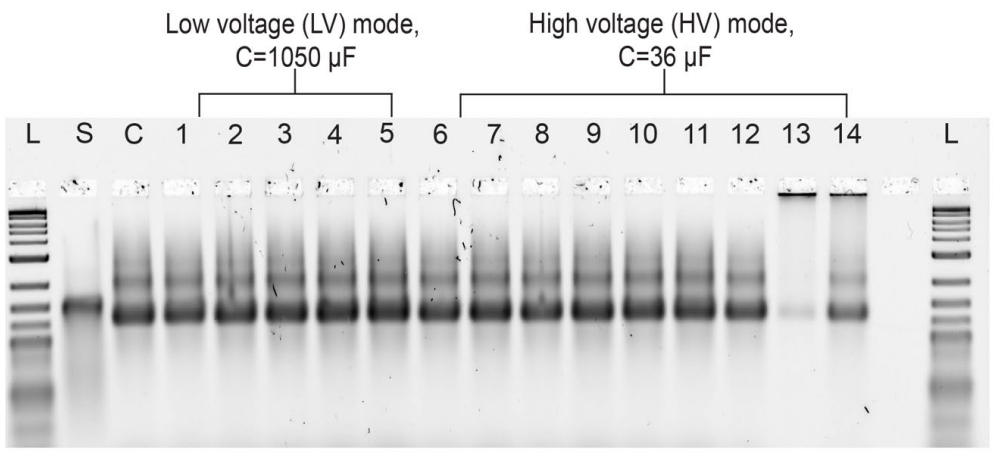

B

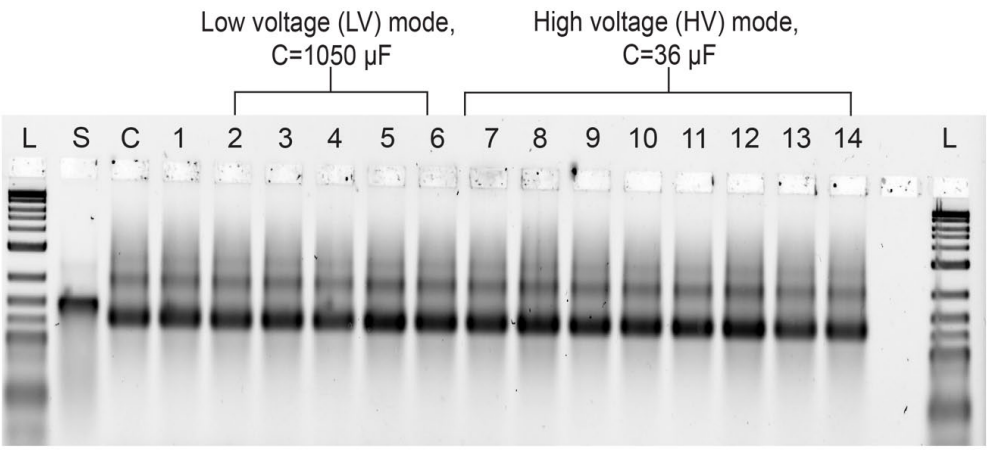

Figure S11. Agarose gel electrophoresis showing the stability Spd-nanostructures (42HB) diluted in RPMI and subjected to a range of electric field strengths both in LV and HV mode. L: 2-Log Ladder, C: Control (No electric field). (A) $0.1 \mathrm{~cm}$ path length. Electric fields corresponding to the numbered lanes are: 1: $100 \mathrm{~V} / \mathrm{cm}, 2: 500 \mathrm{~V} / \mathrm{cm}, 3: 1000 \mathrm{~V} / \mathrm{cm}, 4: 2000$ $\mathrm{V} / \mathrm{cm}, 5: 4000 \mathrm{~V} / \mathrm{cm}$ in the low voltage mode ( $\mathrm{LV}$ mode, $1050 \mu \mathrm{F}$ ) and 6: $500 \mathrm{~V} / \mathrm{cm}, 7: 1000$ $\mathrm{V} / \mathrm{cm}$, 8: $2000 \mathrm{~V} / \mathrm{cm}$, 9: $4000 \mathrm{~V} / \mathrm{cm}, 10: 8000 \mathrm{~V} / \mathrm{cm}, 11: 12000 \mathrm{~V} / \mathrm{cm}, 12: 16000 \mathrm{~V} / \mathrm{cm}, 13$ : $17600 \mathrm{~V} / \mathrm{cm}, 14: 20000 \mathrm{~V} / \mathrm{cm}$ in the high voltage mode (HV mode, $36 \mu \mathrm{F}$ ). At electric field strengths of $\mathrm{E} \approx 17600 \mathrm{~V} / \mathrm{cm}$ and higher, bands appear in the pockets indicating that the stability of the structures is compromised in this case (wells 13, 14). (B) $0.4 \mathrm{~cm}$ path length. Electric fields corresponding to the numbered lanes are: 1: $25 \mathrm{~V} / \mathrm{cm}, 2: 125 \mathrm{~V} / \mathrm{cm}, 3: 250 \mathrm{~V} / \mathrm{cm}, 4: 500$ $\mathrm{V} / \mathrm{cm}, 5: 1000 \mathrm{~V} / \mathrm{cm}$ in the low voltage mode (LV mode, $1050 \mu \mathrm{F}$ ) and 6: $125 \mathrm{~V} / \mathrm{cm}, 7: 250$ $\mathrm{V} / \mathrm{cm}, 8: 500 \mathrm{~V} / \mathrm{cm}$, 9: $1000 \mathrm{~V} / \mathrm{cm}, 10: 2000 \mathrm{~V} / \mathrm{cm}, 11: 3000 \mathrm{~V} / \mathrm{cm}, 12: 4000 \mathrm{~V} / \mathrm{cm}, 13: 4400$ $\mathrm{V} / \mathrm{cm}, 14: 5000 \mathrm{~V} / \mathrm{cm}$ in the high voltage mode ( $\mathrm{HV}$ mode, $36 \mu \mathrm{F}$ ). These observations are also mentioned in a tabulated form in figure S9 B. 
A

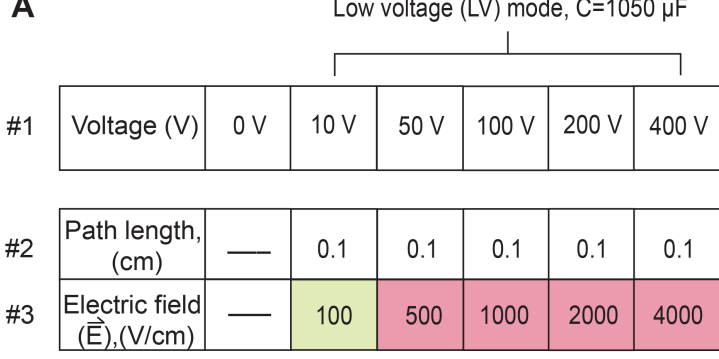

B Low voltage (LV) mode, C=1050 $\mu \mathrm{F}$

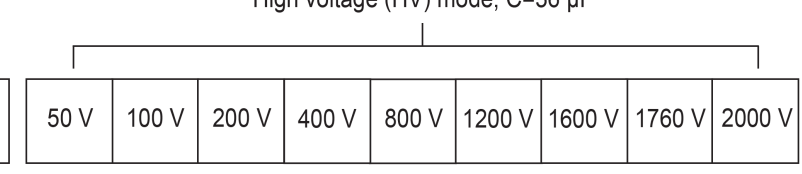

\begin{tabular}{|c|c|c|c|c|c|c|c|c|}
\hline 0.1 & 0.1 & 0.1 & 0.1 & 0.1 & 0.1 & 0.1 & 0.1 & 0.1 \\
\hline 500 & 1000 & 2000 & 4000 & 8000 & 12000 & 16000 & 17600 & 20000 \\
\hline
\end{tabular}

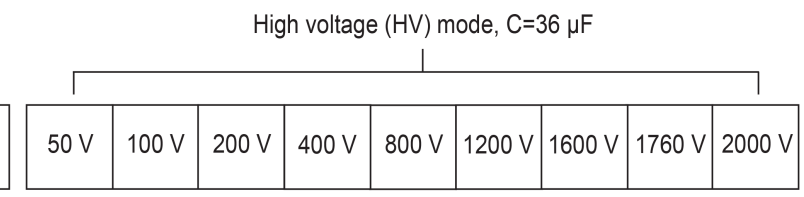

\#2 \begin{tabular}{c|c|c|c|c|c|c|}
\hline $\begin{array}{c}\text { Path length, } \\
(\mathrm{cm})\end{array}$ & - & 0.1 & 0.1 & 0.1 & 0.1 & 0.1 \\
\hline $\begin{array}{c}\text { Electric field } \\
(\vec{E}),(\mathrm{V} / \mathrm{cm})\end{array}$ & - & 100 & 500 & 1000 & 2000 & 4000 \\
\hline
\end{tabular}

\begin{tabular}{|c|c|c|c|c|c|c|c|c|}
\hline 0.1 & 0.1 & 0.1 & 0.1 & 0.1 & 0.1 & 0.1 & 0.1 & 0.1 \\
\hline 500 & 1000 & 2000 & 4000 & 8000 & 12000 & 16000 & 17600 & 20000 \\
\cline { 5 - 8 } & & &
\end{tabular}

DNA nanostructures do not survive

DNA nanostructures survive

Conditions favorable for bacterial transformation

Figure S12. Effect of different electric field strengths on the stability of $\mathrm{Mg}$ and Spdnanostructures diluted in high resistance sample buffers (10\% v/v glycerol in water) and using smaller path length cuvettes $(0.1 \mathrm{~cm})$. (A) Mg-nanostructures. (B) Spd-nanostructures. Voltages, path length and the corresponding electric field strengths analogous to the modes are specified in rows \#1, \#2 and \#3 respectively and the corresponding agarose gels, in (A) and (B). The rows corresponding to electric field strength (\#3) are color coded with respect to survival of nanostructures (pink/green) and conditions favorable for bacterial transformation (orange). 


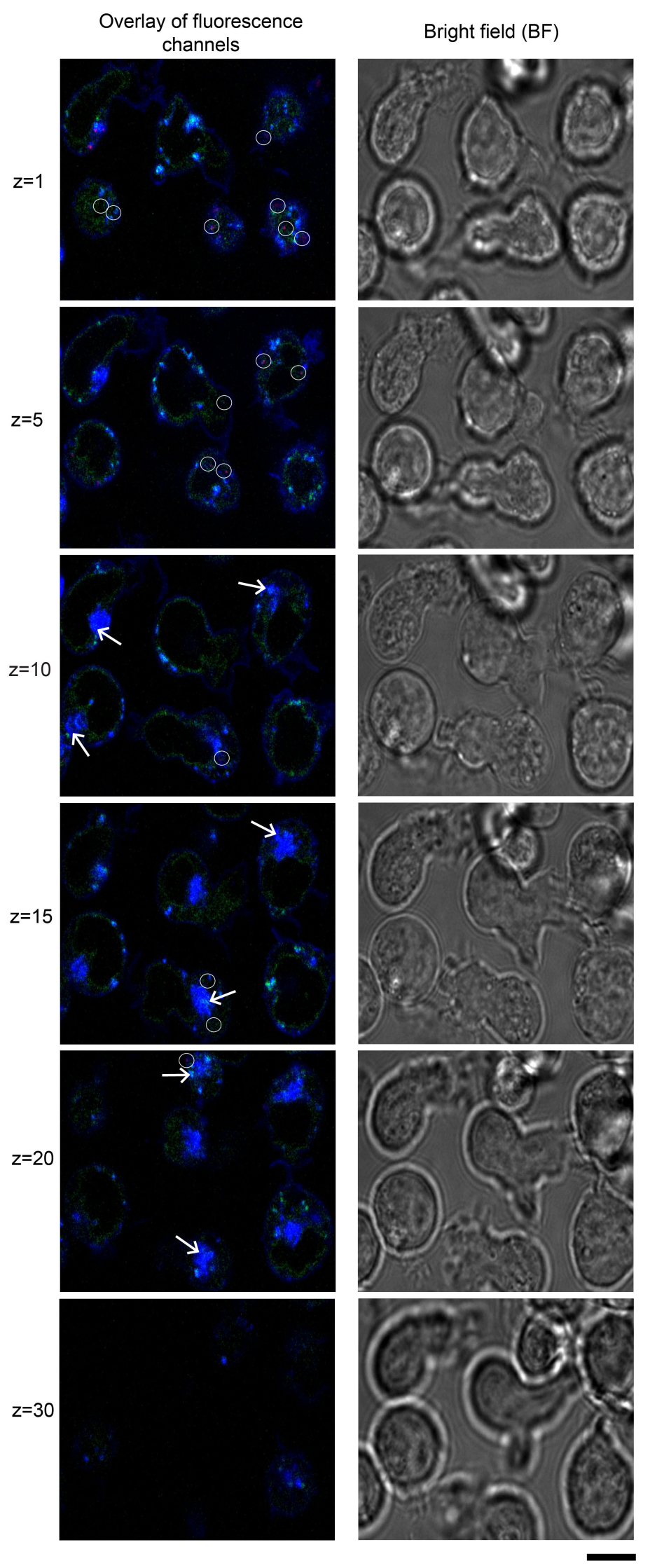

Figure S13. Caption see next page. 
Figure S13 caption. Additional confocal micrographs of Jurkat cells with Atto647N labelled Spd-nanostructures (42HB) internalized via electrotransfection. The cell volume was imaged as z sections each of width $0.5 \mu \mathrm{m}$, accounting for the entire volume of the cell ( $\approx 15 \mu \mathrm{m})$. Confocal micrographs of Jurkat cells showing the overlay of fluorescence channels (green, blue and red) and bright field (BF) taken at different z sections within the cell. The Spd-nanostructures were present at different $\mathrm{z}$ positions inside the cell. The cell organelles lysosome (green) and cell membrane (blue) were stained to confirm the localization of Spd-nanostructures in the cytosol. Blue blobs (shown by white arrows) correspond to the cell membrane which appears at different $\mathrm{z}$ positions for different cells due to variable cell size $(\approx 11-15 \mu \mathrm{m})$ of the cells and differential attachment of cells to the chamber. White circles show the location of Spd-nanostructures inside the cell. Scale bar $10 \mu \mathrm{m}$.
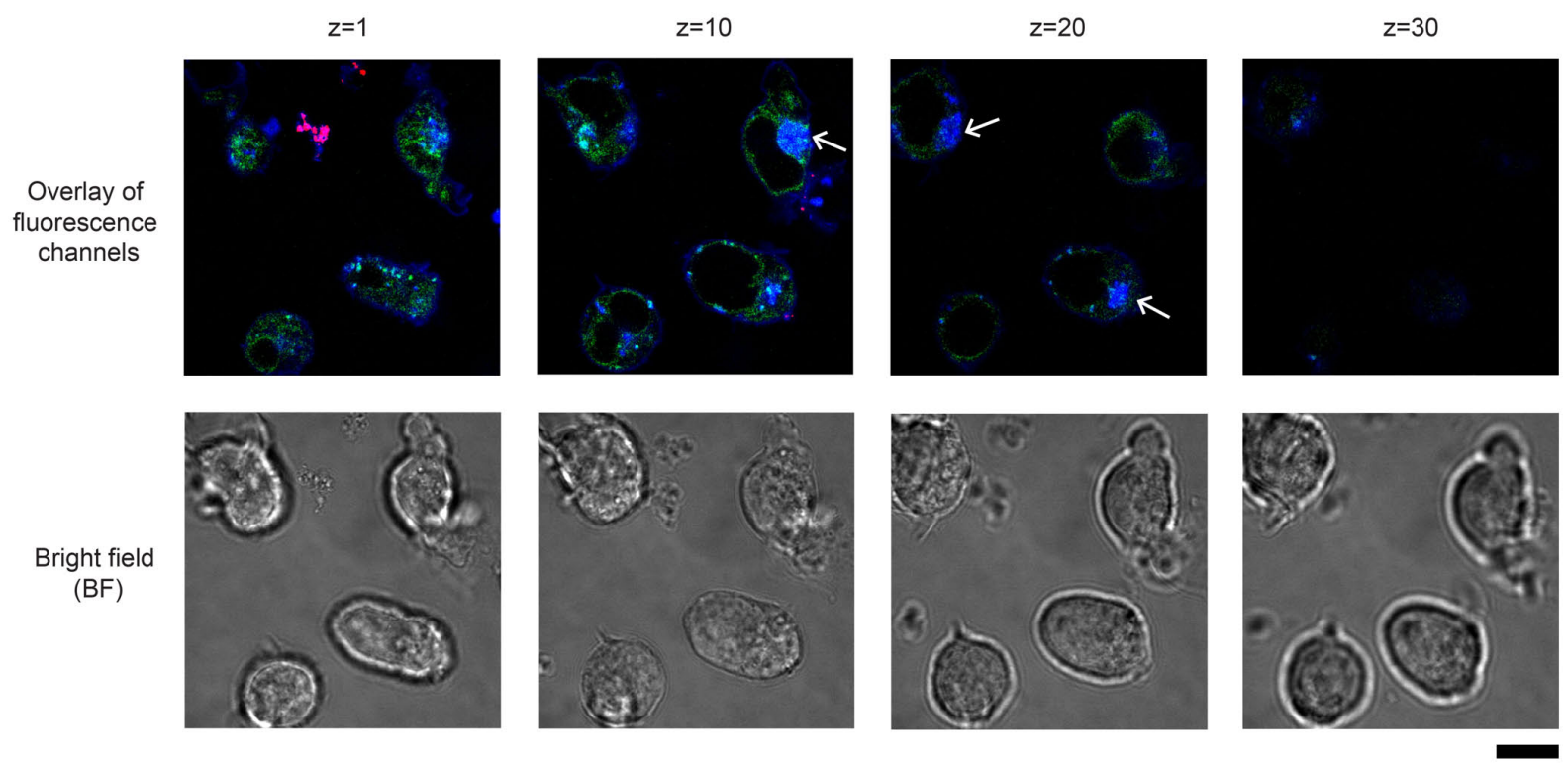

Figure S14. Electroporation experiment with Mg-nanostructures and Jurkat cells. Confocal micrographs of overlay of fluorescence channels (green, blue and red) and bright field (BF) were taken at different $\mathrm{z}$ sections (each $\mathrm{z}=0.5 \mu \mathrm{m}$ ). The cell organelles lysosome (green) and cell membrane (blue) were stained to mark the boundaries of the cell and pinpoint location of the electroporated species (red, Mg-nanostructures). No internalization of Mg-nanostructures was observed, suggesting their disintegration on the application of electric field. The electroporation conditions used were same as for Spd-nanostructures (cf. Supporting information section "Electrotransfection of Spd-nanostructures in Jurkat cells"). Blue blobs (shown by white arrows) correspond to tip the cell membrane which appears at different z positions for different cells due to variable cell size $(\sim 11-15 \mu \mathrm{m})$ of the cells and differential attachment of cells to the chamber. Scale bar $10 \mu \mathrm{m}$. 

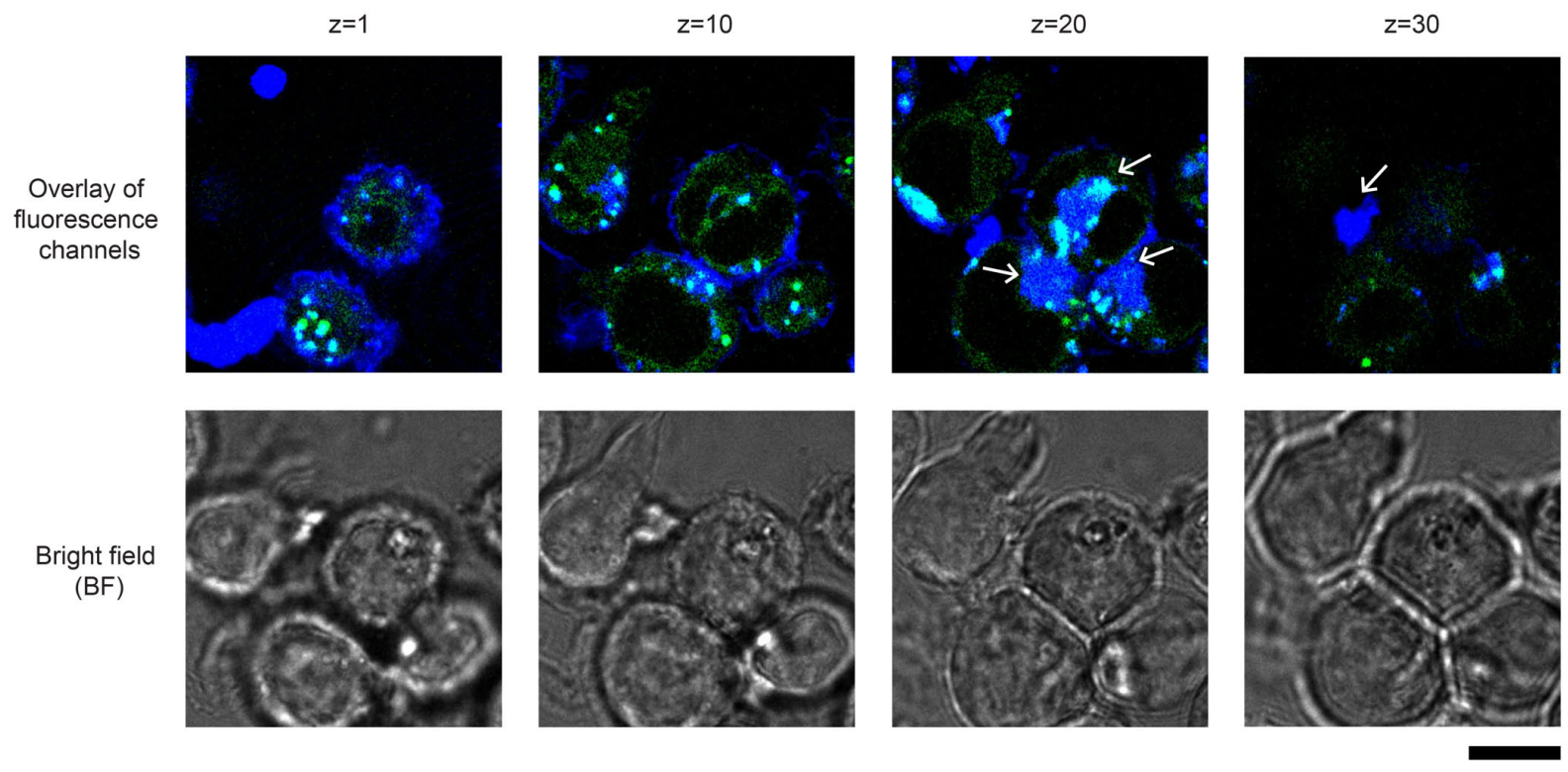

Figure S15. Electroporation experiment with Atto 647N labelled DNA strands in Jurkat cells. Confocal micrographs of overlay of fluorescence channels (green, blue and red) and bright field (BF) were taken at different z positions. The cell organelles lysosome (green) and cell membrane (blue) were stained to mark the boundaries of the cell and pinpoint location of the electroporated species (red, Atto 647N labelled DNA strands). In contrast to experiments with Atto647N labelled 42HB origami structures - carrying multiple co-localized fluorophores -, fluorescence of individual, dispersed DNA strands could not be detected by confocal microscopy. The electroporation conditions used were same as for Spd-nanostructures (cf. Supporting information section "Electrotransfection of Spd-nanostructures in Jurkat cells"). Blue blobs (shown by white arrows) correspond to tip the cell membrane which appears at different $\mathrm{z}$ positions for different cells due to variable cell size $(\sim 11-15 \mu \mathrm{m})$ of the cells and differential attachment of cells to the chamber. Scale bar $10 \mu \mathrm{m}$. 

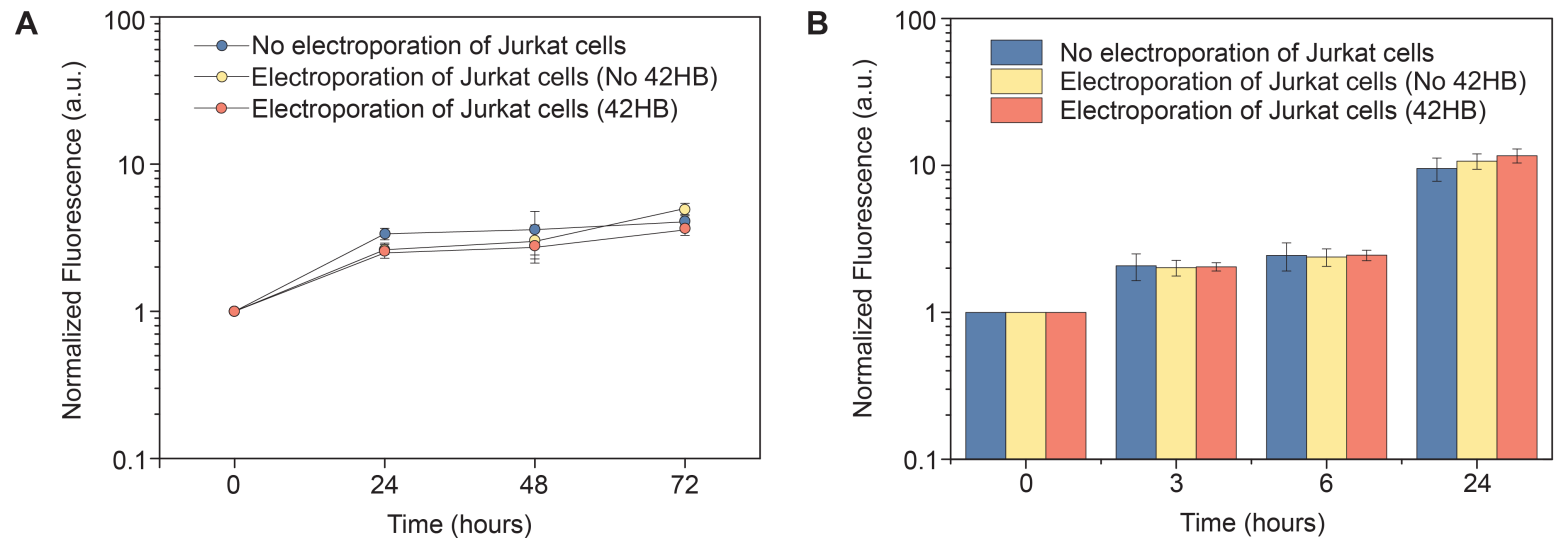

Figure S16. Cell viability assays. (A) CyQuant Direct assay for cell proliferation of cells after electroporation. (B) PrestoBlue cell viability assay. The viability of Jurkat cells (seeded at different densities for the assays- $10^{4}$ cells $/ \mathrm{ml}$ (A) and $10^{6}$ cells $/ \mathrm{ml}$ (B) subjected to different conditions- no electroporation, electroporation without any DNA origami nanostructures, electroporation with $42 \mathrm{HB}$ was analyzed by two different assays working via orthogonal mechanisms. Both assays showed an increase in fluorescence, correlating with the increase in cell number over time, thereby suggesting the viability of Jurkat cells after electroporation without and with $42 \mathrm{HB}$. Error bars represent standard deviation of the mean of $n=3$, triplicates.
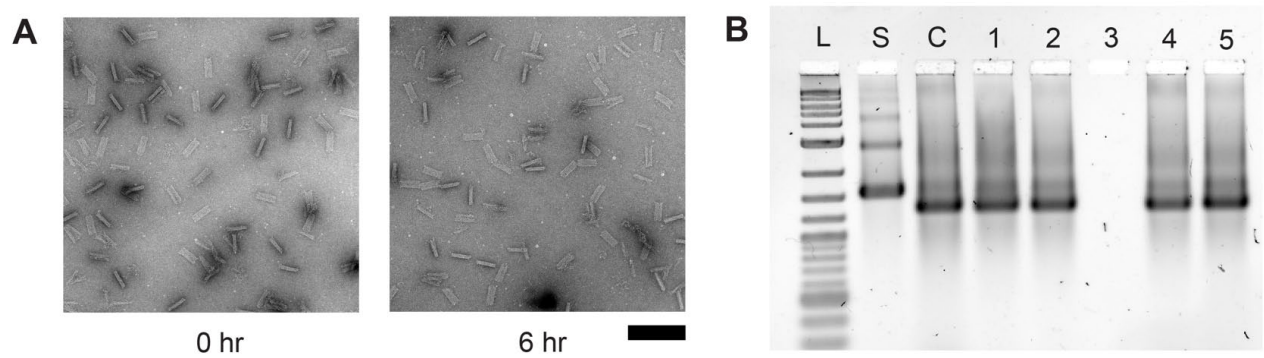

Figure S17. Stability of Spd-nanostructures (42HB) in Jurkat lysate. (A) TEM micrographs of showing the stability of 42HB in Jurkat lysate at 2 different time points ( 0 hour and 6 hour). (B) Agarose gel showing the stability of nanostructures in cell lysates corresponding to different cell densities. L: $2 \log$ ladder C: Control Spd-nanostructures without lysate, 1: 0 hour incubation $\left(10^{3}\right.$ cells $/ \mathrm{ml}), 2: 6$ hour incubation $\left(10^{3}\right.$ cells $\left./ \mathrm{ml}\right) 3:$ Jurkat lysate, 4: 6 hour incubation $\left(10^{4}\right.$ cells $\left./ \mathrm{ml}\right)$, 5: 0 hour incubation $\left(10^{4}\right.$ cells $\left./ \mathrm{ml}\right)$. Scale bar $=200 \mathrm{~nm}$. 
Supporting tables S1-S3 Sequences of DNA staples for different DNA origami nanostructures generated by cadnano software.

Table S1. Sequences of DNA staples for 42HB generated by cadnano software. The staples which are extended at 3 ' for hybridization with dye labelled complementary DNA strand are depicted in red and others in grey, in the cadnano diagram and staple list.

The extension sequence (Ext) is 5'-TTATACATCTATTATACATCTA-3' (22 nt)

The dye is labelled at 5' of sequence 5'-TAGATGTATAATAGATGTATAA-3' (22 nt)

\begin{tabular}{|c|c|c|c|c|}
\hline Start & End & Sequence (5'-3') & Length & Color \\
\hline $0[111]$ & $5[118]$ & $\begin{array}{l}\text { CAGACGTAGGTGAACGGTCGCCCGTCGA } \\
\text { TAAACAGTACCAAGCGCGAAA }\end{array}$ & 49 & \\
\hline $0[167]$ & $1[160]$ & $\begin{array}{l}\text { CATTCCACAGACAGCCCTCATCAAGCAA } \\
\text { GCCCAAT }\end{array}$ & 35 & \\
\hline $0[203]$ & $0[175]$ & TTTTTTTTTTACCAGTACAAACTACAACG & 29 & \\
\hline $0[34]$ & $0[6]$ & AGGAACAACTAAAGGAATTTTTTTTTTTT & 29 & \\
\hline $0[48]$ & $9[55]$ & $\begin{array}{l}\text { GAGTGAGAATCTCCAAGACAGTTAGACT } \\
\text { CCTCAAGTCATAATGACGAGA }\end{array}$ & 49 & \\
\hline $0[69]$ & $5[76]$ & $\begin{array}{l}\text { CAACTTTAAAAGGAGATCGTCTTAGGATT } \\
\text { GAAACAAAAGAATACACTAA }\end{array}$ & 49 & \\
\hline $0[90]$ & $5[97]$ & $\begin{array}{l}\text { TCTGTATCGGTTTAAGTTAAATCAGTACC } \\
\text { TATTTCATCTTTGACCCCCA }\end{array}$ & 49 & \\
\hline $1[112]$ & $12[105]$ & $\begin{array}{l}\text { TTTCTTATTAATAAGACAAAACAGTATGT } \\
\text { TAGCAA }\end{array}$ & 35 & \\
\hline $1[133]$ & $13[132]$ & $\begin{array}{l}\text { GATAGTTCGTTGGGCGATTGATGATTAAG } \\
\text { ACTCCTGCTTTAA }\end{array}$ & 42 & \\
\hline $1[49]$ & $10[63]$ & $\begin{array}{l}\text { AAAAAAAGGAATACTCCACGGAATAAGT } \\
\text { TTATTTTTCAGTTG }\end{array}$ & 42 & \\
\hline
\end{tabular}




\begin{tabular}{|c|c|c|c|}
\hline $1[6]$ & $2[6]$ & $\begin{array}{l}\text { TTTTTTTTTTGCGAATAATAATTAGCAAC } \\
\text { GGCTATTTTTTTTTT }\end{array}$ & 44 \\
\hline $1[70]$ & $12[63]$ & $\begin{array}{l}\text { GCCTTTAAGATTCAGTCACAAAACATATA } \\
\text { AAAGAA }\end{array}$ & 35 \\
\hline $1[91]$ & $12[84]$ & $\begin{array}{l}\text { TCAGCTTACAACATATATGGTAAATACAT } \\
\text { ACATAA }\end{array}$ & 35 \\
\hline $10[160]$ & $13[160]$ & $\begin{array}{l}\text { GAGCCAGATATTGAGAATACCCAAAAGA } \\
\text { GACGAAGCCCTTTT }\end{array}$ & 42 \\
\hline $10[181]$ & $12[175]$ & ACTTGAGTAAAGGTCGAGGAA & 21 \\
\hline $10[200]$ & $1[203]$ & $\begin{array}{l}\text { TTTTTTTTTTCCGTCACCGCTGAGTTTCGT } \\
\text { CTTTTTTTTTT }\end{array}$ & 41 \\
\hline $10[48]$ & $2[35]$ & $\begin{array}{l}\text { CACATTCTTGCCCTCATGACAAGAACCGA } \\
\text { GACTTTCATCGGA }\end{array}$ & 42 \\
\hline $11[2]$ & $10[21]$ & $\begin{array}{l}\text { TTTTTTTTTTATTACGAGGCATAGTACAT } \\
\text { AACG }\end{array}$ & 33 \\
\hline $11[28]$ & $12[6]$ & $\begin{array}{l}\text { AGAGCAATAAAAACCAAAATAGCGAGTT } \\
\text { TTTTTTTT }\end{array}$ & 36 \\
\hline $12[167]$ & $8[168]$ & $\begin{array}{l}\text { AATAACGCGGAAATTATTCATCCATTTGC } \\
\text { ATTACCCATCGAT }\end{array}$ & 42 \\
\hline $12[203]$ & $11[200]$ & $\begin{array}{l}\text { TTTTTTTTTTCCAGAAGGAAACGAATTAT } \\
\text { CATTTTTTTTTT }\end{array}$ & 41 \\
\hline $12[48]$ & $26[49]$ & $\begin{array}{l}\text { TTACCAGGAGGGGGAAATATCGTCAAAA } \\
\text { ATGAAAACCTAATTCTGTAATGGATTGA }\end{array}$ & 56 \\
\hline $13[105]$ & $18[112]$ & $\begin{array}{l}\text { TTCATTGCTATTATCGGGAGACCAGTTAA } \\
\text { AGTACGGTGTCTGTAGTTTG }\end{array}$ & 49 \\
\hline $13[112]$ & $25[111]$ & $\begin{array}{l}\text { AATCCCCAAATCACATCATAACGCAAGA } \\
\text { CAAAGAATGTTAAA }\end{array}$ & 42 \\
\hline $13[6]$ & $14[6]$ & $\begin{array}{l}\text { TTTTTTTTTTAGGCTTTTGCAAGCTTCAAA } \\
\text { GCGATTTTTTTTTT }\end{array}$ & 44 \\
\hline
\end{tabular}




\begin{tabular}{|c|c|c|c|}
\hline $13[63]$ & $18[70]$ & $\begin{array}{l}\text { TTAGACTAGAGGAACTTTACAATAAGAA } \\
\text { GAATATAATGCTGTGTTTAGC }\end{array}$ & 49 \\
\hline $13[70]$ & $25[69]$ & $\begin{array}{l}\text { GGATAGCTTAATGCACCGTGTAGTTAATT } \\
\text { TCATCTATTGTAA }\end{array}$ & 42 \\
\hline $13[84]$ & $18[91]$ & $\begin{array}{l}\text { ACTGCGGCGGATTGAAAACAGATATTAT } \\
\text { CATGTTTTAAATATTTCGCAA }\end{array}$ & 49 \\
\hline $13[91]$ & $25[90]$ & $\begin{array}{l}\text { AATCGTCTCAACCGTAAATAAAAACTTTT } \\
\text { TCAAATTTAAAAT }\end{array}$ & 42 \\
\hline $14[132]$ & $17[146]$ & $\begin{array}{l}\text { AAAATCACTGAACAGTCTTTCCATTCCAT } \\
\text { ATAACAGTTGATT }\end{array}$ & 42 \\
\hline $14[203]$ & $9[200]$ & $\begin{array}{l}\text { TTTTTTTTTTCAATAATAAGAGAGGCCGG } \\
\text { AАTTTTTTTTTT }\end{array}$ & 41 \\
\hline $14[41]$ & $0[35]$ & $\begin{array}{l}\text { GCGTTTTATAAGGCAACTAATCGTTGAAA } \\
\text { ATAGAA }\end{array}$ & 35 \\
\hline $15[133]$ & $35[139]$ & $\begin{array}{l}\text { AAGTCAGCAATAAATGAGTAAGTCTGGC } \\
\text { AGGAACGCGAACTCGAACCAC }\end{array}$ & 49 \\
\hline $15[147]$ & $19[160]$ & $\begin{array}{l}\text { TTGAGCGATCTTACTTAGCGAACCTCCCC } \\
\text { TAAGAATTCATCG }\end{array}$ & 42 \\
\hline $15[6]$ & $16[6]$ & $\begin{array}{l}\text { TTTTTTTTTTACCAGACCGGAACTTTAATT } \\
\text { GCTCTTTTTTTTTT }\end{array}$ & 44 \\
\hline $16[146]$ & $2[133]$ & $\begin{array}{l}\text { CAACGCTCCACCAGGACAGAAAGGTGTA } \\
\text { GACAACAACCATCG }\end{array}$ & 42 \\
\hline $16[160]$ & $26[147]$ & $\begin{array}{l}\text { ATCCTGACTAATATGTAATTCATGTAATA } \\
\text { TAGCTTAGATTAT }\end{array}$ & 42 \\
\hline $16[167]$ & $2[147]$ & $\begin{array}{l}\text { CAATTTTCCGCCGCAGCAGCAAGGTTTAC } \\
\text { ACCCTCATTTTCAGGGATAT }\end{array}$ & 49 \\
\hline $16[203]$ & $7[200]$ & $\begin{array}{l}\text { TTTTTTTTTTATTAGTTGCTATGGTTGAGG } \\
\text { CTTTTTTTTTT }\end{array}$ & 41 \\
\hline $16[34]$ & $21[34]$ & AGAGTACGCAAACTATTAAGCTGTACCA & 28 \\
\hline
\end{tabular}




\begin{tabular}{|c|c|c|c|}
\hline $16[41]$ & $4[35]$ & $\begin{array}{l}\text { GGATTAGCAAGAGTAATCTAACTTTGAA } \\
\text { AGAGGACAAGGCACTGAGGAA }\end{array}$ & 49 \\
\hline $17[119]$ & $2[112]$ & $\begin{array}{l}\text { GAAGTTTCAGAGCCGAGCCACAGCGTCA } \\
\text { GATATAAATAACCGATATATT }\end{array}$ & 49 \\
\hline $17[175]$ & $33[181]$ & $\begin{array}{l}\text { TTTGAAGATAGAAGGCGCCCAAGATTTT } \\
\text { GTAAATCGGTCAGTAATATCA }\end{array}$ & 49 \\
\hline $17[56]$ & $2[49]$ & $\begin{array}{l}\text { AATTGCTACGATTTGAACCAGCATCTTTA } \\
\text { GAAGGAACCCTCAGCAGCGA }\end{array}$ & 49 \\
\hline $17[6]$ & $19[34]$ & $\begin{array}{l}\text { TTTTTTTTTTCTTTTGATAAGAGGTCATTC } \\
\text { ATCAATAATCATA }\end{array}$ & 43 \\
\hline $17[77]$ & $2[70]$ & $\begin{array}{l}\text { AGCTCAATTATCCCCCGCCTCAGCCCCCG } \\
\text { TTTTGCGGCCGCTTTTGCGG }\end{array}$ & 49 \\
\hline $17[98]$ & $2[91]$ & $\begin{array}{l}\text { GCAACTACAAAATACCCTCAGTTCATCGA } \\
\text { TAAGTGTGAGGCTTGCAGGG }\end{array}$ & 49 \\
\hline $18[200]$ & $17[203]$ & $\begin{array}{l}\text { TTTTTTTTTTAAATCAGATCCTTAAATCAA } \\
\text { GTTTTTTTTTT }\end{array}$ & 41 \\
\hline $19[112]$ & $33[118]$ & $\begin{array}{l}\text { CCAAGTAATAACGGAATTACCCACTAAC } \\
\text { ACGAATA }\end{array}$ & 35 \\
\hline $19[147]$ & $28[133]$ & $\begin{array}{l}\text { TTTTATTCGCGAGGCGTTCCCAATTCTGA } \\
\text { ACAAGCCCTTTTA }\end{array}$ & 42 \\
\hline $19[2]$ & $20[2]$ & $\begin{array}{l}\text { TTTTTTTTTTGCATTAACACCTCAGAGCTT } \\
\text { TTTTTTTT }\end{array}$ & 38 \\
\hline $19[49]$ & $30[49]$ & $\begin{array}{l}\text { AGCATGTATTTCAATTACCTGCGGCACCG } \\
\text { CTTCTGTGCCAAG }\end{array}$ & 42 \\
\hline $19[70]$ & $33[76]$ & $\begin{array}{l}\text { AATCGGCTCGCGCACATCAAGTTGAGGA } \\
\text { GACAGTG }\end{array}$ & 35 \\
\hline $19[91]$ & $33[97]$ & $\begin{array}{l}\text { ATTCCAATTTGAATTACATTTAGCCGTCG } \\
\text { TAAGCA }\end{array}$ & 35 \\
\hline $2[203]$ & $1[181]$ & TTTTTTTTTTCTCAGAACCGCCACCCTCAC & 36 \\
\hline
\end{tabular}




\begin{tabular}{|c|c|c|c|}
\hline & & GTAACA & \\
\hline $2[34]$ & $10[28]$ & ACGAGGGTTTTTCAGCAGATA & 21 \\
\hline $20[167]$ & $1[167]$ & $\begin{array}{l}\text { AGGTAAACAGAGAGATAGCAAGTAGCAC } \\
\text { GGAATTAAGGAACC }\end{array}$ & 42 \\
\hline $20[200]$ & $15[203]$ & $\begin{array}{l}\text { TTTTTTTTTTAGAGAATATTTGAGTTAAG } \\
\text { CСTTTTTTTTTT }\end{array}$ & 41 \\
\hline $21[168]$ & $17[167]$ & $\begin{array}{l}\text { GAGGCATCGACAAACATTACCGCTTATCC } \\
\text { GGTATTGACTTGC }\end{array}$ & 42 \\
\hline $21[2]$ & $22[2]$ & $\begin{array}{l}\text { TTTTTTTTTTATAAAGCTAGCCTGAGAGT } \\
\text { TTTTTTTTT }\end{array}$ & 38 \\
\hline $21[21]$ & $18[2]$ & $\begin{array}{l}\text { AATCGGTAATAAAGTCCAATATCTACTAA } \\
\text { TAGTAGTATTTTTTTTTT }\end{array}$ & 47 \\
\hline $22[104]$ & $10[105]$ & $\begin{array}{l}\text { ATGATATATAAATAACGTAGATTACCAG } \\
\text { CGCCAAAAACGAAC }\end{array}$ & 42 \\
\hline $22[111]$ & $18[98]$ & $\begin{array}{l}\text { CATCAATAACCCTCAACGCGCTATTAAAA } \\
\text { CCATTAGATACAT }\end{array}$ & 42 \\
\hline $22[125]$ & $10[126]$ & $\begin{array}{l}\text { GACAGTCCTCAAATTATTACGGGGCGAC } \\
\text { ATTCAACAAGAAAA }\end{array}$ & 42 \\
\hline $22[132]$ & $18[119]$ & $\begin{array}{l}\text { GGCCGGACAATGCCCAACATGCATCGAG } \\
\text { CGAACGAGTAGATT }\end{array}$ & 42 \\
\hline $22[160]$ & $25[160]$ & $\begin{array}{l}\text { ACAACGCATACAAATTGGGTTATATAAC } \\
\text { AAAAGACGCTGAGA }\end{array}$ & 42 \\
\hline $22[181]$ & $24[175]$ & AATTGAGAAGCCAACTTTTTA & 21 \\
\hline $22[200]$ & $13[203]$ & $\begin{array}{l}\text { TTTTTTTTTTGTAGGGCTTCGAACAAAGT } \\
\text { TATTTTTTTTTT }\end{array}$ & 41 \\
\hline $22[41]$ & $11[48]$ & $\begin{array}{l}\text { TACAAAGTTTGCCAACGACGACACTATC } \\
\text { ATAACCC }\end{array}$ & 35 \\
\hline $22[48]$ & $15[41]$ & AGAGATCTATGACCTAAGAATTAGCAAA & 35 \\
\hline
\end{tabular}




\begin{tabular}{|c|c|c|c|}
\hline & & CCAACAG & \\
\hline $22[83]$ & $10[84]$ & $\begin{array}{l}\text { TGATAAAGTCCAATAGGTGGCTCAATAG } \\
\text { AAAATTCTATTACA }\end{array}$ & 42 \\
\hline $22[90]$ & $18[77]$ & $\begin{array}{l}\text { TTCTAGCCGCAAGGTAGATAACCTTATCA } \\
\text { TGGTCAATAACCT }\end{array}$ & 42 \\
\hline $23[2]$ & $22[21]$ & $\begin{array}{l}\text { TTTTTTTTTTTCTGGAGCAAACAAGAGGT } \\
\text { CATT }\end{array}$ & 33 \\
\hline $23[28]$ & $24[6]$ & $\begin{array}{l}\text { GAATCGAATATGTACCCCGGTTGATATTT } \\
\text { TTTTTTT }\end{array}$ & 36 \\
\hline $23[63]$ & $18[56]$ & $\begin{array}{l}\text { AATACCGCGGAGAGCGGGAGAAAATAAT } \\
\text { AATCAATTATATTTTCATTTG }\end{array}$ & 49 \\
\hline $24[146]$ & $13[146]$ & $\begin{array}{l}\text { TATATGTGTATCATATGCGTTCAGATTCA } \\
\text { AAAGGGAGAAAAC }\end{array}$ & 42 \\
\hline $24[203]$ & $23[200]$ & $\begin{array}{l}\text { TTTTTTTTTTCTGAGAGACTACCGCTCAA } \\
\text { CATTTTTTTTTT }\end{array}$ & 41 \\
\hline $24[62]$ & $26[42]$ & $\begin{array}{l}\text { TCTGACCTAAATAACTAGCATATTGTATC } \\
\text { CGTAAT }\end{array}$ & 35 \\
\hline $25[6]$ & $26[6]$ & $\begin{array}{l}\text { TTTTTTTTTTATCAGAAAAGCCTGGTGTA } \\
\text { GATGGTTTTTTTTTT }\end{array}$ & 44 \\
\hline $26[111]$ & $30[112]$ & $\begin{array}{l}\text { CAACATTTCATTTGATTCGCCTTGGGAAG } \\
\text { GGCGATAAGGCGA }\end{array}$ & 42 \\
\hline $26[118]$ & $37[111]$ & $\begin{array}{l}\text { CTTTCATTCAGCTCGTGTAATTCGCCATG } \\
\text { TAATAAAAGGGACGAATCGG }\end{array}$ & 49 \\
\hline $26[181]$ & $25[203]$ & $\begin{array}{l}\text { AGAATCCATTTATCAAAATCATAGGTTTT } \\
\text { TTTTTTT }\end{array}$ & 36 \\
\hline $26[203]$ & $21[200]$ & $\begin{array}{l}\text { TTTTTTTTTTTAATTTTCCCTTCCAGTAAT } \\
\text { ATTTTTTTTTT }\end{array}$ & 41 \\
\hline $26[34]$ & $34[28]$ & GTCACGTCCAAAAACGTAATC & 21 \\
\hline
\end{tabular}




\begin{tabular}{|c|c|c|c|}
\hline $26[69]$ & $30[70]$ & $\begin{array}{l}\text { TGGGAACAAACAAAGAGGCGAAAACCA } \\
\text { GGCAAAGCACGTTGT }\end{array}$ & 42 \\
\hline $26[76]$ & $37[69]$ & $\begin{array}{l}\text { TTCTCCGACGTTAACCTCGATTGGCTATT } \\
\text { GACCTGAAAGCGTCCGCTTT }\end{array}$ & 49 \\
\hline $26[90]$ & $30[91]$ & $\begin{array}{l}\text { AACAACCAATTAATACCAAGTGCCATTC } \\
\text { AGGCTGCGCCAGGG }\end{array}$ & 42 \\
\hline $26[97]$ & $37[90]$ & $\begin{array}{l}\text { AGCGAGTTCGCATTTAAGCTACGAACTGC } \\
\text { CAACAGAGATAGATGTCGTG }\end{array}$ & 49 \\
\hline $27[133]$ & $37[146]$ & $\begin{array}{l}\text { CAGTACATATCTAACGGCTGATTTTCTTT } \\
\text { TTGCGTATTGGGC }\end{array}$ & 42 \\
\hline $27[147]$ & $30[154]$ & $\begin{array}{l}\text { ATATATGATACAGTGGTTAGAACCTACCA } \\
\text { CTTCTG }\end{array}$ & 35 \\
\hline $27[6]$ & $28[6]$ & $\begin{array}{l}\text { TTTTTTTTTTGCGCATCGTAACGTATCGGC } \\
\text { CTCATTTTTTTTTT }\end{array}$ & 44 \\
\hline $28[160]$ & $38[147]$ & $\begin{array}{l}\text { GATGAATTGAGTGATTGAAAGGCATCAC } \\
\text { CTGGTAATATCCCA }\end{array}$ & 42 \\
\hline $28[203]$ & $19[200]$ & $\begin{array}{l}\text { TTTTTTTTTTAAGAAATTGCGTATAGCAA } \\
\text { GCTTTTTTTTTT }\end{array}$ & 41 \\
\hline $28[34]$ & $37[34]$ & $\begin{array}{l}\text { GACGACACGTGCATGTCAACCTCTAATCA } \\
\text { TCCTGTGAGCTAA }\end{array}$ & 42 \\
\hline $29[119]$ & $14[119]$ & $\begin{array}{l}\text { CGGTGCGAGAAACACCGCACTTTCAGCT } \\
\text { GAACACCGGTCTTT }\end{array}$ & 42 \\
\hline $29[133]$ & $20[140]$ & $\begin{array}{l}\text { TCGCTATTACGCAGAACAGTAAAGCCGT } \\
\text { ACGACGA }\end{array}$ & 35 \\
\hline $29[175]$ & $31[181]$ & TGCACGTAATCCTGTCAGATG & 21 \\
\hline $29[56]$ & $15[62]$ & $\begin{array}{l}\text { GTGCCGGATTATTCAGAAACCATCCCATT } \\
\text { AGCAGC }\end{array}$ & 35 \\
\hline $29[6]$ & $30[28]$ & $\begin{array}{l}\text { TTTTTTTTTTGGAAGATCGCACTCCAGCC } \\
\text { GCCAGGG }\end{array}$ & 36 \\
\hline
\end{tabular}




\begin{tabular}{|c|c|c|c|}
\hline $29[77]$ & $14[77]$ & $\begin{array}{l}\text { GCCATTCTACAAAATGTCTTTGTCCTGAT } \\
\text { AACATACATCAAA }\end{array}$ & 42 \\
\hline $29[98]$ & $14[98]$ & $\begin{array}{l}\text { GCAACTGTGATTGCGAACGGGCTGTTTAC } \\
\text { ATTAGAAGTCAGA }\end{array}$ & 42 \\
\hline $3[147]$ & $7[160]$ & $\begin{array}{l}\text { TCACCGTTAAGTTTAAGCCAGAATGGAA } \\
\text { ACAAATAACCAGAG }\end{array}$ & 42 \\
\hline $3[6]$ & $4[6]$ & $\begin{array}{l}\text { TTTTTTTTTTCAGAGGCTTTGATTAAACG } \\
\text { GGTAATTTTTTTTTT }\end{array}$ & 44 \\
\hline $30[200]$ & $29[203]$ & $\begin{array}{l}\text { TTTTTTTTTTCATCAATATAAAACAGAAA } \\
\text { TATTTTTTTTTT }\end{array}$ & 41 \\
\hline $30[41]$ & $17[41]$ & $\begin{array}{l}\text { AGGAGAAAGCTTTCAGGGGACCAGGCAA } \\
\text { GGCAACGAGCTGAAAAGGTGGTTTGCGG }\end{array}$ & 56 \\
\hline $31[112]$ & $33[125]$ & $\begin{array}{l}\text { TTATCATGCGCGTAGAGGCCACCGAGTA } \\
\text { GTGAGACTAGGGGC }\end{array}$ & 42 \\
\hline $31[154]$ & $33[167]$ & $\begin{array}{l}\text { TTATCATGCGTAACCGTTGTAGCAATACG } \\
\text { GCCTTGCTTGCTG }\end{array}$ & 42 \\
\hline $31[161]$ & $13[167]$ & $\begin{array}{l}\text { CATATTCTCAACAGATAACCTCATAGCGT } \\
\text { TAGGCAATATTTATAAGAAA }\end{array}$ & 49 \\
\hline $31[168]$ & $19[167]$ & $\begin{array}{l}\text { CTGATTAATTGTTTGGATTATATATCAAA } \\
\text { ACGTCATAGGAAT }\end{array}$ & 42 \\
\hline $31[2]$ & $32[2]$ & $\begin{array}{l}\text { TTTTTTTTTTTGGTTGTGAAATGTCCCGTT } \\
\text { TTTTTTTT }\end{array}$ & 38 \\
\hline $31[28]$ & $39[34]$ & CGCACGAGCCCGAGAAATCCC & 21 \\
\hline $31[70]$ & $33[83]$ & $\begin{array}{l}\text { TCCTTTGTCCTCGTGAACGGTACGCCAGG } \\
\text { AGAGAGCGGCCCT }\end{array}$ & 42 \\
\hline $31[91]$ & $33[104]$ & $\begin{array}{l}\text { ATTTTAAACGAGCAAGAAGTGTTTTTATT } \\
\text { GCCCTTACTCGTC }\end{array}$ & 42 \\
\hline $32[146]$ & $20[147]$ & $\begin{array}{l}\text { GGAAGGTTAAATCAAATTCGCTGTGTAG } \\
\text { GTAAAACTGTCCAG }\end{array}$ & 42 \\
\hline
\end{tabular}




\begin{tabular}{|c|c|c|c|}
\hline $32[153]$ & $31[139]$ & $\begin{array}{l}\text { GAATTGAAGCGGAAAATAATGGACAGCT } \\
\text { GGCGAAAAAACCAC }\end{array}$ & 42 \\
\hline $32[200]$ & $27[204]$ & $\begin{array}{l}\text { TTTTTTTTTTTCAATATCTGTCGCTATTAA } \\
\text { TTTTTTTTTTTT }\end{array}$ & 42 \\
\hline $33[2]$ & $34[2]$ & $\begin{array}{l}\text { TTTTTTTTTTCCAAAATAATAGCTGTTTTT } \\
\text { TTTTTTTT }\end{array}$ & 38 \\
\hline $33[21]$ & $30[2]$ & $\begin{array}{l}\text { CCCCGCTTTATGACATTCATGTGGATGTT } \\
\text { CTTCTAAGTTTTTTTTTT }\end{array}$ & 47 \\
\hline $33[42]$ & $21[41]$ & $\begin{array}{l}\text { GCTCGCCAATAGTGCTGAATTCTGCCAGG } \\
\text { GGATAGAAAACAT }\end{array}$ & 42 \\
\hline $34[104]$ & $23[111]$ & $\begin{array}{l}\text { CAGGGCTAAATTTTCGCGAGAGAATAAA } \\
\text { CACCGGA }\end{array}$ & 35 \\
\hline $34[125]$ & $23[132]$ & $\begin{array}{l}\text { CTGGTTGATTTTTTATCCAATTTACTAGA } \\
\text { AAAAGC }\end{array}$ & 35 \\
\hline $34[167]$ & $22[168]$ & $\begin{array}{l}\text { GCAAATGAGAGTCAGCTTAGGTTCTTACC } \\
\text { AGTATAAATCGCC }\end{array}$ & 42 \\
\hline $34[200]$ & $36[175]$ & $\begin{array}{l}\text { TTTTTTTTTTCAGTGCCACGCTGAGATTA } \\
\text { ACACTTTTGAC }\end{array}$ & 40 \\
\hline $34[83]$ & $23[90]$ & $\begin{array}{l}\text { CTTGTTATATTTTGATATTTTGATAAATA } \\
\text { AGGCGT }\end{array}$ & 35 \\
\hline $35[105]$ & $30[98]$ & $\begin{array}{l}\text { TAAAACAGAGTAAAGGTGGGCAACTAAT } \\
\text { AGTAACATTAAGTTGGGTAAC }\end{array}$ & 49 \\
\hline $35[126]$ & $30[119]$ & $\begin{array}{l}\text { ACCGAACTGACCTCCTTGAATAATATCTA } \\
\text { ACAAAGGGGGGATGTGCTGC }\end{array}$ & 49 \\
\hline $35[2]$ & $34[21]$ & $\begin{array}{l}\text { TTTTTTTTTTCCTGTGTGAAATTGTTATGG } \\
\text { TCA }\end{array}$ & 33 \\
\hline $35[28]$ & $36[6]$ & $\begin{array}{l}\text { ATCCGCTTAAAGTGTAAAGCCTGGGGTTT } \\
\text { TTTTTTT }\end{array}$ & 36 \\
\hline $35[63]$ & $30[56]$ & TTTTGAAAAAGACGTGATACCTTTAGAAT & 49 \\
\hline
\end{tabular}




\begin{tabular}{|c|c|c|c|}
\hline & & ATTAAAAAAACGACGGCCAG & \\
\hline $35[84]$ & $30[77]$ & $\begin{array}{l}\text { TAATGCGCGTGGTGGCCATCTAATAGATG } \\
\text { TTATTATTTTCCCAGTCACG }\end{array}$ & 49 \\
\hline $36[146]$ & $25[146]$ & $\begin{array}{l}\text { ATTGGCACAGCAGAAGATAAATAAAATC } \\
\text { ATTTCTCCCATCAA }\end{array}$ & 42 \\
\hline $36[203]$ & $35[200]$ & $\begin{array}{l}\text { TTTTTTTTTTGAAATACCTACACGCCTGC } \\
\text { AATTTTTTTTTT }\end{array}$ & 41 \\
\hline $37[161]$ & $32[168]$ & $\begin{array}{l}\text { CCGCCAGCGTCTGATGAGGCGGTCAGTA } \\
\text { GCCAGCAAACCTCATGGCAAA }\end{array}$ & 49 \\
\hline $37[35]$ & $34[42]$ & $\begin{array}{l}\text { CTCACATGGAAGCACACAATTCCACACA } \\
\text { ACCGAGC }\end{array}$ & 35 \\
\hline $37[56]$ & $39[55]$ & $\begin{array}{l}\text { TCACTGCAAGAATACGTGGATACGAGCC } \\
\text { TAATTGCCCCAGCATCAGGCCGATTAAA }\end{array}$ & 56 \\
\hline $37[6]$ & $38[6]$ & $\begin{array}{l}\text { TTTTTTTTTTTGCCTAATGAGTTTGATGGT } \\
\text { GGTTTTTTTTTTTT }\end{array}$ & 44 \\
\hline $38[111]$ & $41[125]$ & $\begin{array}{l}\text { AGCTGATAATCAGTCTATGGTCCCTAAAG } \\
\text { GGAGCCCCCGATT }\end{array}$ & 42 \\
\hline $38[181]$ & $37[203]$ & $\begin{array}{l}\text { GAACTCAAACAGGAAAAACGCTCATGTT } \\
\text { TTTTTTTT }\end{array}$ & 36 \\
\hline $38[203]$ & $33[200]$ & $\begin{array}{l}\text { TTTTTTTTTTGCCTGAGTAGAAAACCCTC } \\
\text { AАTTTTTTTTTT }\end{array}$ & 41 \\
\hline $38[69]$ & $41[83]$ & $\begin{array}{l}\text { AAGCGGTTAGACAGTAGAATCCAACGTC } \\
\text { AAAGGGCGAAAAAC }\end{array}$ & 42 \\
\hline $38[90]$ & $41[104]$ & $\begin{array}{l}\text { TGGCCCTAATCCTGCGTATAACGTCTATC } \\
\text { AACTAAATCGGAA }\end{array}$ & 42 \\
\hline $39[147]$ & $40[161]$ & $\begin{array}{l}\text { AATTAACCACCACAGGCGAACGTGGCGA } \\
\text { GAAAGGAACGCTGC }\end{array}$ & 42 \\
\hline $39[35]$ & $23[48]$ & $\begin{array}{l}\text { TTATAAAGGCGAAATATTTACTCGAATTC } \\
\text { AGGAAGGTCAATCTGAACGGTAATCGT }\end{array}$ & 56 \\
\hline
\end{tabular}




\begin{tabular}{|c|c|c|c|}
\hline $39[56]$ & $25[62]$ & $\begin{array}{l}\text { GGGATTTCCACGCTGACTCTAGAGGATCT } \\
\text { ATTTAA }\end{array}$ & 35 \\
\hline $39[6]$ & $40[6]$ & $\begin{array}{l}\text { TTTTTTTTTTCCGAAATCGGCAATAGGGT } \\
\text { TGAGTTTTTTTTTTT }\end{array}$ & 44 \\
\hline $4[104]$ & $14[91]$ & $\begin{array}{l}\text { TTAATGCCCCCTGCCAGGCGGGCATTTTG } \\
\text { TTTAATAGCAAAG }\end{array}$ & 42 \\
\hline $4[125]$ & $14[112]$ & $\begin{array}{l}\text { TAACAGTGCCCGTAGAGGGTTGACTGTAT } \\
\text { TGTGAAACCCTGA }\end{array}$ & 42 \\
\hline $4[160]$ & $14[147]$ & $\begin{array}{l}\text { CTGGTAAACTCAGGCCGTAATATCACCAT } \\
\text { AGCTATCTTACGA }\end{array}$ & 42 \\
\hline $4[203]$ & $3[181]$ & $\begin{array}{l}\text { TTTTTTTTTTGTCATACATGGCTTTTGATC } \\
\text { ACССTC }\end{array}$ & 36 \\
\hline $4[34]$ & $22[28]$ & $\begin{array}{l}\text { GTTTCCAGGACTAAGATATTCTCAGTGAA } \\
\text { ATTCGAAAGAAGTGCTATCA }\end{array}$ & 49 \\
\hline $4[55]$ & $6[49]$ & $\begin{array}{l}\text { ATTAAGAGGCTGCACAACCTAAAACGAA } \\
\text { TGACCAA }\end{array}$ & 35 \\
\hline $4[83]$ & $14[70]$ & $\begin{array}{l}\text { GGAACCTATTATTCTAGCGGGTTATTAGT } \\
\text { AGTAAAAAGATTA }\end{array}$ & 42 \\
\hline $40[104]$ & $21[104]$ & $\begin{array}{l}\text { TGCTTTGAAGTTTGAGATTAGAACAATTA } \\
\text { AATGTGTTTTTAG }\end{array}$ & 42 \\
\hline $40[125]$ & $21[125]$ & $\begin{array}{l}\text { CTACAGGTTTGCGGTTAGGAGTTTTTTAT } \\
\text { AGCCAGTTAAATG }\end{array}$ & 42 \\
\hline $40[139]$ & $33[153]$ & $\begin{array}{l}\text { CGCTTAACTGTCCATCACGCAGGGTGGTC } \\
\text { GCATTTCACATAA }\end{array}$ & 42 \\
\hline $40[181]$ & $39[203]$ & $\begin{array}{l}\text { CAAGTGTATTAGTAATAACATCACTTTTT } \\
\text { TTTTTTT }\end{array}$ & 36 \\
\hline $40[203]$ & $31[200]$ & $\begin{array}{l}\text { TTTTTTTTTTCTAGGGCGCTGGATGGCAA } \\
\text { TTTTTTTTTTTT }\end{array}$ & 41 \\
\hline $40[83]$ & $21[83]$ & CGTGCTTCCCGAACAATACATAAAACAA & 42 \\
\hline
\end{tabular}




\begin{tabular}{|c|c|c|c|}
\hline & & CGTCGGAATTTCAA & \\
\hline $41[133]$ & $31[146]$ & TGACGGGGAAAGCCCCCGCCGCAGAAGG & 28 \\
\hline $41[175]$ & $41[203]$ & AAAGCGAAAGGAGCGGGCGTTTTTTTTTT & 29 \\
\hline $41[35]$ & $30[42]$ & $\begin{array}{l}\text { AGAGTCCAAGAATACTTAAGTGTCCTACC } \\
\text { TTTCTC }\end{array}$ & 35 \\
\hline $41[49]$ & $21[62]$ & $\begin{array}{l}\text { AAGAACGTGGACTCAGAGCGGCAACTCG } \\
\text { GTATTAGGATGATGAAACGGCACTTTTG }\end{array}$ & 56 \\
\hline $41[6]$ & $41[34]$ & TTTTTTTTTTGTTGTTCCAGTTTGGAACA & 29 \\
\hline $5[133]$ & $3[139]$ & $\begin{array}{l}\text { GATTTGTATCATTATAACGGGGTCAGTGC } \\
\text { CGGAAT }\end{array}$ & 35 \\
\hline $5[175]$ & $21[181]$ & $\begin{array}{l}\text { ATTTACCGATTGGCGACAGGATTTGCACA } \\
\text { CAAGAAAAAGTACTTTCGAG }\end{array}$ & 49 \\
\hline $5[6]$ & $7[34]$ & $\begin{array}{l}\text { TTTTTTTTTTAATACGTAATGCCACTACG } \\
\text { AGATGAACCTTCAT }\end{array}$ & 43 \\
\hline $6[118]$ & $21[118]$ & $\begin{array}{l}\text { CGACCTGACCCTCATAATTTGATTAACTA } \\
\text { ATGCAGATATATT }\end{array}$ & 42 \\
\hline $6[146]$ & $16[133]$ & ATCGCCTGATAAATAGAGCCGAACGAGC & 28 \\
\hline $6[200]$ & $5[203]$ & $\begin{array}{l}\text { TTTTTTTTTTAGGTCAGACGTTCCAGTAA } \\
\text { GCTTTTTTTTTT }\end{array}$ & 41 \\
\hline $6[76]$ & $21[76]$ & $\begin{array}{l}\text { TCAATCAACCGGAAAATCCAAGAGAGAA } \\
\text { ACAAGAAAGCCTTT }\end{array}$ & 42 \\
\hline $6[97]$ & $21[97]$ & $\begin{array}{l}\text { GCCGGAAGCCGCCAAACAGCCGGAAGCG } \\
\text { TCAACAAATAAAAA }\end{array}$ & 42 \\
\hline $7[168]$ & $4[161]$ & $\begin{array}{l}\text { CAGCATTCTTGATATTCACAAAGCGCAGG } \\
\text { AGTGTA }\end{array}$ & 35 \\
\hline $7[2]$ & $8[2]$ & $\begin{array}{l}\text { TTTTTTTTTTCGCATAGGCAAATCAACGT } \\
\text { TTTTTTTTT }\end{array}$ & 38 \\
\hline
\end{tabular}




\begin{tabular}{|c|c|c|c|}
\hline $7[49]$ & $18[49]$ & $\begin{array}{l}\text { ATCACCGTTTGTTTAACGTCAATGGCTTA } \\
\text { GAGCTTGGGCGCG }\end{array}$ & 42 \\
\hline $8[139]$ & $23[139]$ & $\begin{array}{l}\text { TCAAGTTTTTAAGATAAATCAACAGTTCT } \\
\text { GAGAAACTGTTTA }\end{array}$ & 42 \\
\hline $8[181]$ & $13[181]$ & ATGAAACATTAGCACAAGAAAAGATAGC & 28 \\
\hline $8[200]$ & $3[203]$ & $\begin{array}{l}\text { TTTTTTTTTTACGTCACCAAGAACCGCCA } \\
\text { CCTTTTTTTTTT }\end{array}$ & 41 \\
\hline $9[105]$ & $5[104]$ & $\begin{array}{l}\text { TTAATCAGCGCGTTAACCGCCCTCCATGT } \\
\text { TACTTAGCGATTA }\end{array}$ & 42 \\
\hline $9[119]$ & $0[112]$ & $\begin{array}{l}\text { TTACCTTATCTACGAACAGCTTTTTGTCG } \\
\text { TCTTTC }\end{array}$ & 35 \\
\hline $9[126]$ & $5[125]$ & $\begin{array}{l}\text { ATGCGATTGCCTTTCACCCTCTGTGTCGA } \\
\text { AATCCGCAAAGTA }\end{array}$ & 42 \\
\hline $9[2]$ & $10[2]$ & $\begin{array}{l}\text { TTTTTTTTTTTAACAAAGCCCAAAAGGAT } \\
\text { TTTTTTTTT }\end{array}$ & 38 \\
\hline $9[21]$ & $6[2]$ & $\begin{array}{l}\text { TGCTCATATTACCCTGGCTGACGGTGTAC } \\
\text { AGACCAGGTTTTTTTTTT }\end{array}$ & 47 \\
\hline $9[77]$ & $0[70]$ & $\begin{array}{l}\text { TTGGGCTGGTAGAAATTGTATGGGATTTT } \\
\text { GCTAAA }\end{array}$ & 35 \\
\hline $9[84]$ & $5[83]$ & $\begin{array}{l}\text { TGAGATGCGGTCATCCTCAGACGAGGCG } \\
\text { CAGACGGAACACTC }\end{array}$ & 42 \\
\hline $0[139]$ & $5[132]$ & $\begin{array}{l}\text { GTAACGATCTAAAGTGATACCCCCACGC } \\
\text { GTATAGCCCTTGAGCAACGGA-Ext }\end{array}$ & 49 \\
\hline $0[174]$ & $5[174]$ & $\begin{array}{l}\text { CCTGTAGCATGTACGAGCCACGTACCGC } \\
\text { GATACAGTCTCTGA-Ext }\end{array}$ & 42 \\
\hline $12[146]$ & $0[140]$ & $\begin{array}{l}\text { ACTGGCAGGGAGGGAAGGTAACATACCA } \\
\text { GTCAGGAGCGCCGAAGTTAGC-Ext }\end{array}$ & 49 \\
\hline $12[174]$ & $17[174]$ & $\begin{array}{l}\text { ACGCAATAGTAAGCCAATGAAATAACCC } \\
\text { CCAGCTAGGGAGGT-Ext }\end{array}$ & 42 \\
\hline
\end{tabular}




\begin{tabular}{|c|c|c|c|}
\hline $14[55]$ & $23[62]$ & $\begin{array}{l}\text { AGACTTCTAATAGTATTTTTGAATTAATG } \\
\text { GTTTGA-Ext }\end{array}$ & 35 \\
\hline $14[62]$ & $0[49]$ & $\begin{array}{l}\text { GCCCGAAAACACCAAGATTTAAGGCTCC } \\
\text { CAACAGTTTCAGCG-Ext }\end{array}$ & 42 \\
\hline $15[140]$ & $6[147]$ & $\begin{array}{l}\text { AGGGTAAATGACCAACTGGCTCATTAAA } \\
\text { CAGTAGCAACCACCAATCCTC-Ext }\end{array}$ & 49 \\
\hline $22[62]$ & $12[49]$ & $\begin{array}{l}\text { GGTAGCTAAAATGTACGCAAAGACACGT } \\
\text {-Ext }\end{array}$ & 28 \\
\hline $24[139]$ & $29[132]$ & $\begin{array}{l}\text { AAATGCTGATGCAAAACCAATCTTCCTGA } \\
\text { TGGAAACATCGGGGGCCTCT-Ext }\end{array}$ & 49 \\
\hline $24[174]$ & $29[174]$ & $\begin{array}{l}\text { ACCTCCGATAGTGATTGAAAATGCTTCTC } \\
\text { AGGTTTAATTATT-Ext }\end{array}$ & 42 \\
\hline $25[49]$ & $35[62]$ & $\begin{array}{l}\text { AAGCAAACCCGGGTACCACAGACAATAT } \\
\text {-Ext }\end{array}$ & 28 \\
\hline $27[42]$ & $37[55]$ & $\begin{array}{l}\text { TTTGAGCAAAAGAAACTTTACCTGGAGT } \\
\text { GGTTTGCGTTGCGC-Ext }\end{array}$ & 42 \\
\hline $31[49]$ & $41[48]$ & $\begin{array}{l}\text { AATTCGAGAGCTAAACAGGAAACTATTA } \\
\text {-Ext }\end{array}$ & 28 \\
\hline $34[160]$ & $37[160]$ & $\begin{array}{l}\text { AAAAATCACAGAGGAATGGATTATTTAC } \\
\text { GCAGAACAATATTA-Ext }\end{array}$ & 42 \\
\hline $36[139]$ & $41[132]$ & $\begin{array}{l}\text { GATTCACCAGTCACGAGGCGGTTCACCA } \\
\text { AAAGAGTTGCGCCGTAGAGCT-Ext }\end{array}$ & 49 \\
\hline $36[174]$ & $41[174]$ & $\begin{array}{l}\text { GCTCAATCCATTGCAACTATCTTCTTTGA } \\
\text { GCGGTCAGGGAAG-Ext }\end{array}$ & 42 \\
\hline $38[118]$ & $35[125]$ & $\begin{array}{l}\text { GGGCAACCCAACGCGCGGGGAACGACCA } \\
\text { TAAAAAT-Ext }\end{array}$ & 35 \\
\hline $38[76]$ & $35[83]$ & $\begin{array}{l}\text { TTGCAGCCCAGTCGGGAAACCACCCTTCT } \\
\text { AGTCTT-Ext }\end{array}$ & 35 \\
\hline $38[97]$ & $35[104]$ & CACCGCCCCAGCTGCATTAATATTCTGGA & 35 \\
\hline
\end{tabular}




\begin{tabular}{|l|l|l|l|}
\hline & \multicolumn{2}{|l|}{ TAGCCC-Ext } & \\
\hline $9[63]$ & $4[56]$ & $\begin{array}{l}\text { GAACGAGCGTTTGCAGCCACCTAAGGGA } \\
\text { ACCGAACAGAGGCATGAAAGT-Ext }\end{array}$ & 49 \\
\hline $9[98]$ & $0[91]$ & $\begin{array}{l}\text { TTCAACTTAACGGAGCTTTCGTAGTAAAT } \\
\text { GAATTT-Ext }\end{array}$ & 35 \\
\hline
\end{tabular}

Table S2. Sequences of DNA staples for tube generated by cadnano software.

\begin{tabular}{|c|c|c|c|c|}
\hline Start & End & Sequence $(5 '-3$ ') & Length & Color \\
\hline $15[252]$ & $12[252]$ & $\begin{array}{l}\text { AGGATTAGGATTAGCGGGGTTTTTTTTTT } \\
\text { TTTAGCGCGTTTTCATCGGCA }\end{array}$ & 50 & \\
\hline $2[41]$ & $3[41]$ & $\begin{array}{l}\text { TAATACATTTGAGGATTTATTTTTTTTTTT } \\
\text { TCATGGAAATACCTACATTT }\end{array}$ & 50 & \\
\hline $6[34]$ & $7[34]$ & $\begin{array}{l}\text { GTAATTTAGGCATTTTTTTTTTTTATGGAA } \\
\text { ACAGTA }\end{array}$ & 36 & \\
\hline $0[41]$ & $1[41]$ & $\begin{array}{l}\text { TTGCAACAGGAAAAACGCTTTTTTTTTTT } \\
\text { TTAATCGGAACCCT }\end{array}$ & 43 & \\
\hline $19[252]$ & $16[252]$ & $\begin{array}{l}\text { ATGAACGGTGTATTTTTTTTTTTTCTTAAA } \\
\text { CAGCTT }\end{array}$ & 36 & \\
\hline $29[252]$ & $26[252]$ & $\begin{array}{l}\text { TGCAGCAAGCGGTCCATTTTTTTTTTTATT } \\
\text { ACGCCAGCTGGC }\end{array}$ & 42 & \\
\hline $10[34]$ & $11[34]$ & $\begin{array}{l}\text { TATGGTTTATTTTTTTTTTTTAAAATAAAC } \\
\text { AGC }\end{array}$ & 33 & \\
\hline $24[48]$ & $25[48]$ & $\begin{array}{l}\text { CGAGTAACAACCCGTCGGATTTTTTTTTT } \\
\text { TTAGAACCCTCATATATTTTA }\end{array}$ & 50 & \\
\hline $25[245]$ & $22[245]$ & $\begin{array}{l}\text { ACGGTAATCGTAAAACTAGTTTTTTTTTT } \\
\text { TTAATAACCTGTTTAGCTATA }\end{array}$ & 50 & \\
\hline $20[33]$ & $21[41]$ & $\begin{array}{l}\text { CGGATTTTTTTTTTTTAAGAAAAATCTAC } \\
\text { GTT }\end{array}$ & 32 & \\
\hline
\end{tabular}




\begin{tabular}{|c|c|c|c|}
\hline $3[259]$ & $0[259]$ & $\begin{array}{l}\text { CAGCAGAAGTTTTTTTTTTTTCTAAACAG } \\
\text { G }\end{array}$ & 30 \\
\hline $14[34]$ & $15[34]$ & CCACAGACATTTTTTTTTTTTATAAATCCT & 30 \\
\hline $26[41]$ & $27[41]$ & $\begin{array}{l}\text { CCGGAAGCATAATTTTTTTTTTTTTTCTCC } \\
\text { GTGGGA }\end{array}$ & 36 \\
\hline $16[41]$ & $17[34]$ & $\begin{array}{l}\text { AAATACGTAATGCCACTTTTTTTTTTTTGC } \\
\text { CCTCATA }\end{array}$ & 37 \\
\hline $23[245]$ & $20[245]$ & $\begin{array}{l}\text { ATACATTTCGCAAATGGTCTTTTTTTTTTT } \\
\text { TATGTTTAGACTGGATAGCG }\end{array}$ & 50 \\
\hline $8[34]$ & $9[34]$ & $\begin{array}{l}\text { TTTGCCAGTTACTTTTTTTTTTTTGAGGCA } \\
\text { TTTTCG }\end{array}$ & 36 \\
\hline $21[252]$ & $18[252]$ & $\begin{array}{l}\text { GGTAATAGTAAATTTTTTTTTTTTCAGAC } \\
\text { CAGGCGC }\end{array}$ & 36 \\
\hline $13[252]$ & $10[252]$ & $\begin{array}{l}\text { TGCCTTTAGCGTCAGACTGTTTTTTTTTTT } \\
\text { TATCTTACCGAAGCCCTTTT }\end{array}$ & 50 \\
\hline $7[259]$ & $4[259]$ & $\begin{array}{l}\text { AAGACAAAGAACTTTTTTTTTTTTTAAAA } \\
\text { CAGA }\end{array}$ & 33 \\
\hline $1[252]$ & $28[252]$ & $\begin{array}{l}\text { AGAATCAGAGCGGGAGTTTTTTTTTTTCG } \\
\text { CTGGTTTGCCCCAG }\end{array}$ & 43 \\
\hline $4[41]$ & $5[41]$ & $\begin{array}{l}\text { ATTTGAATTACCTTTTTTATTTTTTTTTTTT } \\
\text { GAAGTATTAGACTTTACAA }\end{array}$ & 50 \\
\hline $9[259]$ & $6[259]$ & $\begin{array}{l}\text { GTACCGCACTCATTTTTTTTTTTTGCGAG } \\
\text { AAAACTT }\end{array}$ & 36 \\
\hline $17[252]$ & $14[252]$ & $\begin{array}{l}\text { CGAGGTGAATTTTTTTTTTTTTTTTTTTGC } \\
\text { TCAGTACCAGGCG }\end{array}$ & 43 \\
\hline $28[41]$ & $29[41]$ & $\begin{array}{l}\text { CGTAAAGCACTATTTTTTTTTTTTAGTGTA } \\
\text { AAGCCT }\end{array}$ & 36 \\
\hline $18[41]$ & $19[41]$ & $\begin{array}{l}\text { CAGTCAGGACGTTGGGTTTTTTTTTTTTTA } \\
\text { CGAAGGCACCAACC }\end{array}$ & 44 \\
\hline
\end{tabular}




\begin{tabular}{|c|c|c|c|}
\hline $11[259]$ & $8[259]$ & $\begin{array}{l}\text { ATAGCAATAGCTTTTTTTTTTTTTTCGAGA } \\
\text { ACAAGC }\end{array}$ & 36 \\
\hline $22[48]$ & $23[48]$ & $\begin{array}{l}\text { CGCAAGGATAAAAATTTTTTTTTTTTTTTT } \\
\text { TAGCAAACTCCAACAGGTCA }\end{array}$ & 50 \\
\hline $5[259]$ & $2[259]$ & ATTTGCACGTTTTTTTTTTTTATAAAACAG & 30 \\
\hline $27[252]$ & $24[245]$ & $\begin{array}{l}\text { GTGCGGGCCTCTTCGCTTTTTTTTTTTTCA } \\
\text { TGTCAATCATATGTACC }\end{array}$ & 47 \\
\hline $12[34]$ & $13[34]$ & $\begin{array}{l}\text { CACAAACAATTTTTTTTTTTTCCAGCGCC } \\
\text { A }\end{array}$ & 30 \\
\hline $17[63]$ & $20[56]$ & $\begin{array}{l}\text { TCTTTCCTCATGAGATACACTTGCGATTA } \\
\text { ACATTAATCGCGTTTTAATT }\end{array}$ & 49 \\
\hline 7[49] & $10[42]$ & $\begin{array}{l}\text { TGTGAGTCATATTTGAATATAGAGCGTCC } \\
\text { AATCCATCACAATCAATAGA }\end{array}$ & 49 \\
\hline $7[245]$ & $0[252]$ & $\begin{array}{l}\text { GCAAATCCAATCGCAATAAAGCAAAATT } \\
\text { AGGTGAGGAACCACAGGCCGA }\end{array}$ & 49 \\
\hline $27[224]$ & $0[217]$ & $\begin{array}{l}\text { GCTGCGCGCGATTAGCCCTTCGGTGGTTC } \\
\text { GAGCACACGGTACGCCAGAA }\end{array}$ & 49 \\
\hline $27[98]$ & $0[91]$ & $\begin{array}{l}\text { GTAACCGCATGGTCTTCCAGTAAAACCG } \\
\text { GGAAGGGGAGTAGAAGAACTC }\end{array}$ & 49 \\
\hline $17[210]$ & $20[203]$ & $\begin{array}{l}\text { CCAAAAGTCGCCCAAATCATACAAGAAC } \\
\text { AAAATAGTCATTGAATCCCCC }\end{array}$ & 49 \\
\hline $17[126]$ & $20[119]$ & $\begin{array}{l}\text { TCAGCGGCGAAAGAGAGATTTAACGAGT } \\
\text { AGATACATATAGTCAGAAGCA }\end{array}$ & 49 \\
\hline $17[42]$ & $20[34]$ & $\begin{array}{l}\text { TAACGATACGGGTATAAAACGATTATAC } \\
\text { AATAAAACAAAGCGAACCAGAC }\end{array}$ & 50 \\
\hline $17[133]$ & $10[140]$ & $\begin{array}{l}\text { AGTGAGAATAGAAACGCCACCGGTCAGT } \\
\text { GAACCGCGAATTAGACGCAGT }\end{array}$ & 49 \\
\hline $17[238]$ & $10[245]$ & $\begin{array}{l}\text { TATCAGCTTGCTTTGATAAGTCAAGAGAT } \\
\text { TTTCGGTCAAGTTTAAGAAA }\end{array}$ & 49 \\
\hline
\end{tabular}




\begin{tabular}{|c|c|c|c|}
\hline $17[105]$ & $20[98]$ & $\begin{array}{l}\text { GCTAAACGGGTAGCAGCGCGAGAGATGG } \\
\text { AATACCATTGCATCAAAAAGA }\end{array}$ & 49 \\
\hline $17[217]$ & $10[224]$ & $\begin{array}{l}\text { GAGCCTTTAATTGTTGATATATATTAAGT } \\
\text { TAGCGTCCGTAATCGAACAA }\end{array}$ & 49 \\
\hline $7[224]$ & $0[231]$ & $\begin{array}{l}\text { TAACTATATGTAAATTCAGGTAAGGGTTC } \\
\text { ACCGCCTCGCCATGACAGGA }\end{array}$ & 49 \\
\hline $27[168]$ & $20[175]$ & $\begin{array}{l}\text { CTTTCCGGCACCGCAACGTTAATTTTTGT } \\
\text { CCAATACTGGAAGGAAAACG }\end{array}$ & 49 \\
\hline $17[154]$ & $10[161]$ & $\begin{array}{l}\text { CTAAAGGAATTGCGCAGAACCTGCCCGT } \\
\text { CCCTCAGCCAGTAGGCATGAT }\end{array}$ & 49 \\
\hline $7[77]$ & $0[84]$ & $\begin{array}{l}\text { TCGTCGCTATTAATTGATGAATAATTTTA } \\
\text { AATATCTCACCAGAAACTAT }\end{array}$ & 49 \\
\hline $7[175]$ & $10[168]$ & $\begin{array}{l}\text { ATAGGTCGCGTTAATCCCATCTATTCTAT } \\
\text { CAGAGGACCCAAAAGAACTG }\end{array}$ & 49 \\
\hline $27[147]$ & $20[154]$ & $\begin{array}{l}\text { GGAAGATCGCACTCTTCGCATTTAATGCG } \\
\text { GCAAAGTATGCAATCAAAAA }\end{array}$ & 49 \\
\hline $7[112]$ & $10[105]$ & $\begin{array}{l}\text { GAAAACACGTTATATCAGCTAATCAAGA } \\
\text { AGAATAAACATACATAAAGGT }\end{array}$ & 49 \\
\hline $27[42]$ & $20[49]$ & $\begin{array}{l}\text { ACAAACGGCGGATTATGTGAGAATGCAA } \\
\text { ATTTCAAGGATTAGCGAGCTT }\end{array}$ & 49 \\
\hline $27[56]$ & $0[49]$ & $\begin{array}{l}\text { GACCGTACAATTCCGTGAGCTAGTTTTTA } \\
\text { TTTAGAGAACAATATTACCG }\end{array}$ & 49 \\
\hline $17[70]$ & $10[77]$ & $\begin{array}{l}\text { AGACGTTAGTAAATCGTAACATCCAGTA } \\
\text { CCAGCATAAATATTAAAGACA }\end{array}$ & 49 \\
\hline $27[105]$ & $20[112]$ & $\begin{array}{l}\text { TGCATCTGCCAGTTTAGGAACAAATCAC } \\
\text { AAAGCTACTTAATTAAGCGGA }\end{array}$ & 49 \\
\hline $27[77]$ & $0[70]$ & $\begin{array}{l}\text { GTTGGTGTGTGAAAGCGTTGCCGTGAAC } \\
\text { GCCGGCGCGGCCTTGCTGGTA }\end{array}$ & 49 \\
\hline $27[203]$ & $0[196]$ & AAAGCGCAGGGTTTTGAGACGATCCCTT & 49 \\
\hline
\end{tabular}




\begin{tabular}{|c|c|c|c|}
\hline & & CGCGTACAAGTGTTTTTATAA & \\
\hline $17[196]$ & $10[203]$ & $\begin{array}{l}\text { CCAAAAAAAAGGCTTAGGTGTTATTATTT } \\
\text { AATCAAATGAAACAACCGAG }\end{array}$ & 49 \\
\hline $7[35]$ & $0[42]$ & $\begin{array}{l}\text { CATAAATCAATATACAATTTCACAATTCC } \\
\text { AATAGATGACGCTCCAGCCA }\end{array}$ & 49 \\
\hline $7[238]$ & $10[231]$ & $\begin{array}{l}\text { TGCTGATTAGTTAACAAGAACTTCATCGA } \\
\text { TAAGAGAGTAAGCAGATAGC }\end{array}$ & 49 \\
\hline $7[70]$ & $10[63]$ & $\begin{array}{l}\text { CTGTAAATAGGGCTGGTAAAGCTGAATC } \\
\text { TTTTGTTCCACGGAATAAGTT }\end{array}$ & 49 \\
\hline $7[154]$ & $10[147]$ & $\begin{array}{l}\text { AATAGTGCGGAATCTCCTGAATTTAGCG } \\
\text { AACTGAATAAGACTCCTTATT }\end{array}$ & 49 \\
\hline $7[161]$ & $0[168]$ & $\begin{array}{l}\text { AATTTATCAAAATCGGATTCGTATCAGAG } \\
\text { CTGAACGGCACAGAGAGTCT }\end{array}$ & 49 \\
\hline $17[231]$ & $20[224]$ & $\begin{array}{l}\text { ATCGGTTCGCCGACACCAACTCTTCATCA } \\
\text { AAAGAACTGCGGAATCGTCA }\end{array}$ & 49 \\
\hline $17[91]$ & $10[98]$ & $\begin{array}{l}\text { CTGTATGGGATTTTGCCCAATCTTTTGAG } \\
\text { AACCACATTAAAGGGCAACA }\end{array}$ & 49 \\
\hline $7[203]$ & $0[210]$ & $\begin{array}{l}\text { TCCGGCTTAGGTTGATATACAGATTATAG } \\
\text { CTGAGACGAACTGTCCTGAG }\end{array}$ & 49 \\
\hline $17[35]$ & $10[35]$ & $\begin{array}{l}\text { GTTAGCGTAGCATTCATTAAATGATATTA } \\
\text { AGACAAAAATTCA }\end{array}$ & 42 \\
\hline 7[98] & $0[105]$ & $\begin{array}{l}\text { CCCTTAGAATCCTTAATTACCCATTATCG } \\
\text { GAATTGATAAAAGCTTGCCT }\end{array}$ & 49 \\
\hline $27[231]$ & $20[238]$ & $\begin{array}{l}\text { AACTGTTGGGAAGGCCGGTTGTCGATGA } \\
\text { TTTTCATCCATTAGTCCAATA }\end{array}$ & 49 \\
\hline $7[196]$ & $10[189]$ & $\begin{array}{l}\text { TTTAACCACCGACCTGTAGAAAGATATA } \\
\text { ATATCAGGAAACGCAATAATA }\end{array}$ & 49 \\
\hline $27[182]$ & $0[175]$ & $\begin{array}{l}\text { TTCTGGTTTGTAAAGGTGGTTTAGCCCGG } \\
\text { CTTAATGGCCACCGAGTAAA }\end{array}$ & 49 \\
\hline
\end{tabular}




\begin{tabular}{|c|c|c|c|}
\hline $17[168]$ & $20[161]$ & $\begin{array}{l}\text { AATAATAGGGAGTTACCTGCTGCTCATTA } \\
\text { ACACTAAGAATGACCATAAA }\end{array}$ & 49 \\
\hline $27[63]$ & $20[70]$ & $\begin{array}{l}\text { ATGGGATAGGTCACGCCAGCTGTAGGTA } \\
\text { ACTTTTGTGCTCCTTTCAAAT }\end{array}$ & 49 \\
\hline $27[245]$ & $0[238]$ & $\begin{array}{l}\text { GCGATCGGAAAGGGAGAGAGTCAGGCG } \\
\text { ACCTCGTTTTAAAGGGATTTTA }\end{array}$ & 49 \\
\hline $7[133]$ & $10[126]$ & $\begin{array}{l}\text { ATTAAGAAAGCCTGTGTTTATCGGGAGG } \\
\text { AGCGCATATGTTAGCAAACGT }\end{array}$ & 49 \\
\hline $17[189]$ & $20[182]$ & $\begin{array}{l}\text { AAAATCTATTCGGTCGGAACGCCAAATC } \\
\text { TACCAGACTTTAAACAGTTCA }\end{array}$ & 49 \\
\hline $27[119]$ & $0[112]$ & $\begin{array}{l}\text { TGAGGGGTACCGAGTGCCAGCGACTCCA } \\
\text { AGCGGGCTAGTAATAACATCA }\end{array}$ & 49 \\
\hline $27[210]$ & $20[217]$ & $\begin{array}{l}\text { CATTCGCCATTCAGCCCAAAAAGTCTGG } \\
\text { GAAAAGGGAACGAGTAAATAT }\end{array}$ & 49 \\
\hline $27[126]$ & $20[133]$ & $\begin{array}{l}\text { ACGACGACAGTATCATCAGCTTCAACCG } \\
\text { AGCAATATGTAGCTTGACTAT }\end{array}$ & 49 \\
\hline $17[112]$ & $10[119]$ & $\begin{array}{l}\text { AACTTTCAACAGTTCCTCATTACTGGTAC } \\
\text { CACCСTTCACCGAAGAAAAT }\end{array}$ & 49 \\
\hline $7[91]$ & $10[84]$ & $\begin{array}{l}\text { TAATTTTTATAAAGCGACGACGCACCCA } \\
\text { AAAATAGTATAAAAGAAACGC }\end{array}$ & 49 \\
\hline $7[56]$ & $0[63]$ & $\begin{array}{l}\text { GAATAACCTTGCTTAACAAAAAATCCTTC } \\
\text { AACTAAGGATTATATATCCA }\end{array}$ & 49 \\
\hline $17[49]$ & $10[56]$ & $\begin{array}{l}\text { CTAAAGTTTTGTCGCAGTACACGCAGTCG } \\
\text { GCAGGTACCGATTTATTTTG }\end{array}$ & 49 \\
\hline $27[84]$ & $20[91]$ & $\begin{array}{l}\text { TAGATGGGCGCATCTTCGCGTTGAGAAA } \\
\text { AAAACATATTTTTGTTAAGAG }\end{array}$ & 49 \\
\hline $17[147]$ & $20[140]$ & $\begin{array}{l}\text { GGAACAACGGGATCTAAATTGTGCCCTG } \\
\text { ATTACGATCAGGTCTTTACCC }\end{array}$ & 49 \\
\hline $27[161]$ & $0[154]$ & CAGCCAGCAAGCTTGGTTTGCGTTGTTCC & 49 \\
\hline
\end{tabular}




\begin{tabular}{|c|c|c|c|}
\hline & & GTAACCGTCCATCACGCAAA & \\
\hline $7[182]$ & $0[189]$ & $\begin{array}{l}\text { TGAGAGACTACCTTTACATCGCAATATAA } \\
\text { AAAATCTGGCTATTCAGTGA }\end{array}$ & 49 \\
\hline $17[84]$ & $20[77]$ & $\begin{array}{l}\text { GAATTTTCTTTGAGTGACCCCTAATCATA } \\
\text { TTCATCGAAGCCCGAAAGAC }\end{array}$ & 49 \\
\hline $27[140]$ & $0[133]$ & $\begin{array}{l}\text { GGCCTCACGACTCTGGCCAACAGTCCAC } \\
\text { AAGTGTATTGTAGCAATACTT }\end{array}$ & 49 \\
\hline $7[119]$ & $0[126]$ & $\begin{array}{l}\text { TAGCGATAGCTTAGCAGAGGCAGAAACC } \\
\text { TTGGCAAACAGAGACTTTGAT }\end{array}$ & 49 \\
\hline $7[217]$ & $10[210]$ & $\begin{array}{l}\text { GGTTATACTAAATTGGCTGTCGCGCCCAA } \\
\text { GAATTGAGTTACCAGAAGGA }\end{array}$ & 49 \\
\hline $27[189]$ & $20[196]$ & $\begin{array}{l}\text { GCCGGAAACCAGGCTAAGCAAGCTATCA } \\
\text { CTAATAGACAGTTGTCAAATG }\end{array}$ & 49 \\
\hline $7[140]$ & $0[147]$ & $\begin{array}{l}\text { CGCTGAGAAGAGTCATACCAAAATTATC } \\
\text { CCTCAATCCTGAAATTAACCG }\end{array}$ & 49 \\
\hline $17[175]$ & $10[182]$ & $\begin{array}{l}\text { ATTTTTTCACGTTGGAGGTTTCCCCCTGG } \\
\text { AGCCACGCAAGGCACGGAAT }\end{array}$ & 49 \\
\hline $29[49]$ & $26[42]$ & CTAATGAACACAACATACGAG & 21 \\
\hline $9[231]$ & $6[224]$ & ATCATTCTTTCATCTTCTGAC & 21 \\
\hline $13[105]$ & $12[112]$ & GTGAATTATCACCGCAGAGCC & 21 \\
\hline $3[238]$ & $2[245]$ & TAAAAATACCGAACGCGGTCA & 21 \\
\hline $23[161]$ & $22[168]$ & CTAAAGTACGGTGTAATCATA & 21 \\
\hline $19[161]$ & $16[154]$ & ATCCGCGAAAGGCCGCTTTTG & 21 \\
\hline $13[168]$ & $12[175]$ & CACCATTACCATTACACCGGA & 21 \\
\hline $29[70]$ & $26[63]$ & ATTAATTTTGTTATCCGCTCA & 21 \\
\hline $3[70]$ & $2[77]$ & TTACATTGGCAGATTTTAGGA & 21 \\
\hline
\end{tabular}




\begin{tabular}{|c|c|c|c|}
\hline $19[77]$ & $16[70]$ & TCATCTTGACTAAAGACTTTT & 21 \\
\hline $9[210]$ & $6[203]$ & AATAATCTAATGGTTTGAAAT & 21 \\
\hline $29[112]$ & $26[105]$ & CCTGTCGCTCGAATTCGTAAT & 21 \\
\hline $11[245]$ & $9[251]$ & $\begin{array}{l}\text { CAAGAAACAATGAAAAGCCGTTTTTATT } \\
\text { GGGTATT }\end{array}$ & 35 \\
\hline $2[223]$ & $4[217]$ & $\begin{array}{l}\text { GTGCCACCTTCTGAATAATGGTTAACGTC } \\
\text { AGATGA }\end{array}$ & 35 \\
\hline $1[210]$ & $29[216]$ & $\begin{array}{l}\text { TATGGTTGCTTTGACCGAAATCGGCAAA } \\
\text { GGCAACA }\end{array}$ & 35 \\
\hline $22[62]$ & $24[56]$ & $\begin{array}{l}\text { AGCCTTTTGCCTGAGTAATGTTTCATCAA } \\
\text { CATTAA }\end{array}$ & 35 \\
\hline $19[182]$ & $16[175]$ & ACTTAGCCGCTGAGGCTTGCA & 21 \\
\hline $2[55]$ & $4[49]$ & $\begin{array}{l}\text { GAGCCGTGACAACTCGTATTATTAATTAC } \\
\text { ATTTAA }\end{array}$ & 35 \\
\hline $9[126]$ & $6[119]$ & ACGCGCCTTTAGTATCATATG & 21 \\
\hline $29[91]$ & $26[84]$ & GCCCGCTATAGCTGTTTCCTG & 21 \\
\hline $23[56]$ & $22[63]$ & AGAGTACCTTTAATCGGGAGA & 21 \\
\hline $2[76]$ & $4[70]$ & $\begin{array}{l}\text { GCACTAATGCCCGAACGTTATACAAACA } \\
\text { TCAAGAA }\end{array}$ & 35 \\
\hline $13[63]$ & $12[70]$ & GAGGGAGGGAAGGTTGACAGG & 21 \\
\hline $13[84]$ & $12[91]$ & GACGGAAATTATTCCACCAGA & 21 \\
\hline $22[188]$ & $24[182]$ & $\begin{array}{l}\text { ATTAACAAGAGATCTACAAAGATATTTA } \\
\text { AATTGTA }\end{array}$ & 35 \\
\hline $22[125]$ & $24[119]$ & $\begin{array}{l}\text { AGAGCATCATCAATATGATATCATTTTTT } \\
\text { AACCAA }\end{array}$ & 35 \\
\hline $22[146]$ & $24[140]$ & AAAATTATTCTAGCTGATAAATAAATTTT & 35 \\
\hline
\end{tabular}




\begin{tabular}{|c|c|c|c|}
\hline & & TGTTAA & \\
\hline $9[252]$ & $6[245]$ & AAACCAATTTCAAATATATTT & 21 \\
\hline $19[98]$ & $16[91]$ & TATACCAAACGGCTACAGAGG & 21 \\
\hline $3[133]$ & $2[140]$ & TAGAACCCTTCTGACAATATC & 21 \\
\hline $23[77]$ & $22[84]$ & TTTGATAAGAGGTCTATGACC & 21 \\
\hline $13[126]$ & $12[133]$ & CTTGAGCCATTTGGCACCCTC & 21 \\
\hline $2[139]$ & $4[133]$ & $\begin{array}{l}\text { TGGTCAGACCAGAAGGAGCGGGTTACAA } \\
\text { AATCGCG }\end{array}$ & 35 \\
\hline $2[160]$ & $4[154]$ & $\begin{array}{l}\text { ATCAAACATCATATTCCTGATCCTGATTG } \\
\text { CTTTGA }\end{array}$ & 35 \\
\hline $2[118]$ & $4[112]$ & $\begin{array}{l}\text { GTTGAAAATTTTGCGGAACAAGAATTATT } \\
\text { CATTTC }\end{array}$ & 35 \\
\hline $2[181]$ & $4[175]$ & $\begin{array}{l}\text { TCACCTTTGATGGCAATTCATGGAGAAAC } \\
\text { AATAAC }\end{array}$ & 35 \\
\hline $29[133]$ & $26[126]$ & ATGAATCAGAGGATCCCCGGG & 21 \\
\hline $12[132]$ & $14[126]$ & $\begin{array}{l}\text { AGAGCCAATAAGTTTTAACGGCTCAGAG } \\
\text { CCACCAC }\end{array}$ & 35 \\
\hline $22[209]$ & $24[203]$ & $\begin{array}{l}\text { AATTCTAGGTCATTGCCTGAGACAGGAA } \\
\text { GATTGTA }\end{array}$ & 35 \\
\hline $1[231]$ & $29[237]$ & $\begin{array}{l}\text { GTATAACGTGCTTTAAATCCTGTTTGATA } \\
\text { CCGCCT }\end{array}$ & 35 \\
\hline $19[245]$ & $16[238]$ & AGGACAGGATACCGATAGTTG & 21 \\
\hline $2[244]$ & $4[238]$ & $\begin{array}{l}\text { GTATTAAAGAACCTACCATATAAATTGC } \\
\text { GTAGATT }\end{array}$ & 35 \\
\hline $12[48]$ & $14[42]$ & $\begin{array}{l}\text { TTGGCCTGCCAGAATGGAAAGAACTACA } \\
\text { ACGCCTG }\end{array}$ & 35 \\
\hline
\end{tabular}




\begin{tabular}{|c|c|c|c|}
\hline $22[167]$ & $24[161]$ & $\begin{array}{l}\text { CAGGCAACGGAGAGGGTAGCTATATTTT } \\
\text { GTTAAAA }\end{array}$ & 35 \\
\hline $13[189]$ & $12[196]$ & CGGAAACGTCACCAAATCACC & 21 \\
\hline $9[189]$ & $6[182]$ & ACGAGCAGTGTGATAAATAAG & 21 \\
\hline $23[182]$ & $22[189]$ & TTTCATTCCATATATAGTAGC & 21 \\
\hline $19[140]$ & $16[133]$ & CGCCTGAGTCACCCTCAGCAG & 21 \\
\hline $23[119]$ & $22[126]$ & GCTGAATATAATGCAAGCCTC & 21 \\
\hline $22[230]$ & $24[224]$ & $\begin{array}{l}\text { GCGAGCTAGCAAACAAGAGAAATAATCA } \\
\text { GAAAAGC }\end{array}$ & 35 \\
\hline $2[97]$ & $4[91]$ & $\begin{array}{l}\text { TTATCTAAAAAGTTTGAGTAATGAGCAA } \\
\text { AAGAAGA }\end{array}$ & 35 \\
\hline $19[119]$ & $16[112]$ & TACAACGCAGCATCGGAACGA & 21 \\
\hline $29[238]$ & $26[231]$ & GGCCCTGGGATGTGCTGCAAG & 21 \\
\hline $13[231]$ & $12[238]$ & CAGTAGCGACAGAATCATAGC & 21 \\
\hline $9[63]$ & $6[56]$ & GACAAAATAATTGAGAATCGC & 21 \\
\hline $12[195]$ & $14[189]$ & $\begin{array}{l}\text { GGAACCACCTATTTCGGAACCATCACCGT } \\
\text { ACTCAG }\end{array}$ & 35 \\
\hline $12[90]$ & $14[84]$ & $\begin{array}{l}\text { GCCGCCGAGCGTCATACATGGAGGAACC } \\
\text { CATGTAC }\end{array}$ & 35 \\
\hline $11[35]$ & $9[41]$ & $\begin{array}{l}\text { CATATTATTTATCCTTTCCAGAGCCTAAA } \\
\text { GCCAGT }\end{array}$ & 35 \\
\hline $3[154]$ & $2[161]$ & GCGTAAGAATACGTCTCAAAT & 21 \\
\hline $19[224]$ & $16[217]$ & CGAACTGAATGACAACAACCA & 21 \\
\hline $22[104]$ & $24[98]$ & $\begin{array}{l}\text { TGTACCAGGCCGGAGACAGTCGCCATCA } \\
\text { AAAATAA }\end{array}$ & 35 \\
\hline
\end{tabular}




\begin{tabular}{|c|c|c|c|}
\hline $9[147]$ & $6[140]$ & AGATAAGATAATTACTAGAAA & 21 \\
\hline $12[216]$ & $14[210]$ & $\begin{array}{l}\text { CTTTTCACTGAAACATGAAAGAGTATAG } \\
\text { CCCGGAA }\end{array}$ & 35 \\
\hline $2[202]$ & $4[196]$ & $\begin{array}{l}\text { GCAAATGATCCTGATTGTTTGGTAACAGT } \\
\text { ACCTTT }\end{array}$ & 35 \\
\hline $23[224]$ & $22[231]$ & TAGATTTAGTTTGATTGGGGC & 21 \\
\hline $19[56]$ & $16[49]$ & CAAAAGAGAAGTTTCCATTAA & 21 \\
\hline $3[49]$ & $2[56]$ & CAATCGTCTGAAATTAGATTA & 21 \\
\hline $29[175]$ & $26[168]$ & GCGCCAGACGACGGCCAGTGC & 21 \\
\hline $13[147]$ & $12[154]$ & AGCCAGCAAAATCAAGCCGCC & 21 \\
\hline $29[217]$ & $26[210]$ & GCTGATTAGTTGGGTAACGCC & 21 \\
\hline $29[154]$ & $26[147]$ & GAGAGGCGCATGCCTGCAGGT & 21 \\
\hline $3[217]$ & $2[224]$ & ATAGCCCTAAAACATGCAACA & 21 \\
\hline $12[237]$ & $14[231]$ & $\begin{array}{l}\text { CCCCTTAAGGCTGAGACTCCTGCCGTCGA } \\
\text { GAGGGT }\end{array}$ & 35 \\
\hline $13[210]$ & $12[217]$ & CATCGATAGCAGCATTGCCAT & 21 \\
\hline $11[224]$ & $9[230]$ & $\begin{array}{l}\text { AGTTAAGCCCAATATAGGAATCATTACCT } \\
\text { TTCCTT }\end{array}$ & 35 \\
\hline $11[56]$ & $9[62]$ & $\begin{array}{l}\text { AATAAGAAACGATTTTACCAACGCTAAC } \\
\text { AAGTACC }\end{array}$ & 35 \\
\hline $9[84]$ & $6[77]$ & GTCCAGACCAACGCTCAACAG & 21 \\
\hline $19[203]$ & $16[196]$ & GACGGTCCGCATAACCGATAT & 21 \\
\hline $12[153]$ & $14[147]$ & $\begin{array}{l}\text { ACCCTCAGCCTTGAGTAACAGGCCACCCT } \\
\text { CAGAAC }\end{array}$ & 35 \\
\hline $29[196]$ & $26[189]$ & TCACCAGTCCCAGTCACGACG & 21 \\
\hline
\end{tabular}




\begin{tabular}{|c|c|c|c|}
\hline $9[42]$ & $6[35]$ & AATAAGAAACAACGCCAACAT & 21 \\
\hline $1[42]$ & $29[48]$ & $\begin{array}{l}\text { AAAGGGAGCCCCCGTGGGGTCGAGGTGC } \\
\text { GGGGTGC }\end{array}$ & 35 \\
\hline $22[83]$ & $24[77]$ & $\begin{array}{l}\text { CTGTAATAAGATTCAAAAGGGCTGGCCT } \\
\text { TCCTGTA }\end{array}$ & 35 \\
\hline $23[140]$ & $22[147]$ & CAACATGTTTTAAAAATTAGC & 21 \\
\hline $3[112]$ & $2[119]$ & GGACATTCTGGCCAATCAACA & 21 \\
\hline $12[69]$ & $14[63]$ & $\begin{array}{l}\text { AGGTTGATCTGAATTTACCGTCTGAGTTT } \\
\text { CGTCAC }\end{array}$ & 35 \\
\hline $21[49]$ & $19[55]$ & $\begin{array}{l}\text { CGAACTAACGGAACTTAAGAACTGGCTC } \\
\text { AAAGAGG }\end{array}$ & 35 \\
\hline $12[111]$ & $14[105]$ & $\begin{array}{l}\text { GCCACCATGATACAGGAGTGTTTCAGGG } \\
\text { ATAGCAA }\end{array}$ & 35 \\
\hline $23[98]$ & $22[105]$ & CGGATGGCTTAGAGAATCGGT & 21 \\
\hline $13[42]$ & $12[49]$ & AAGGGCGACATTCACAGACGA & 21 \\
\hline $9[105]$ & $6[98]$ & AACATGTCAAATTCTTACCAG & 21 \\
\hline $12[174]$ & $14[168]$ & $\begin{array}{l}\text { ACCGCCTATAAACAGTTAATGAGTACCG } \\
\text { CCACССТ }\end{array}$ & 35 \\
\hline $23[203]$ & $22[210]$ & ATTCCCAATTCTGCTGGCATC & 21 \\
\hline $3[175]$ & $2[182]$ & ACAATATTTTTGAATAAAGCA & 21 \\
\hline $9[168]$ & $6[161]$ & AATAATAATAAGAATAAACAC & 21 \\
\hline $3[196]$ & $2[203]$ & TAGTCTTTAATGCGGCCAGCA & 21 \\
\hline $21[91]$ & $19[97]$ & $\begin{array}{l}\text { AGTTGAGATTTAGGTTTAATTTCAACTTC } \\
\text { AGCGAT }\end{array}$ & 35 \\
\hline $3[91]$ & $2[98]$ & TCACACGACCAGTAAGGAAGG & 21 \\
\hline
\end{tabular}




\begin{tabular}{|c|c|c|c|}
\hline $21[196]$ & $19[202]$ & $\begin{array}{l}\text { CGACGATAAAAACCCGGATATTCATTAC } \\
\text { AGGCGCA }\end{array}$ & 35 \\
\hline $21[112]$ & $19[118]$ & $\begin{array}{l}\text { CATTCAACTAATGCAGTAAATTGGGCTTA } \\
\text { ACAAAG }\end{array}$ & 35 \\
\hline $11[182]$ & $9[188]$ & $\begin{array}{l}\text { GTAATTGAGCGCTAGAAGGCTTATCCGG } \\
\text { CTAATTT }\end{array}$ & 35 \\
\hline $1[126]$ & $29[132]$ & $\begin{array}{l}\text { GCTAGGGCGCTGGCTATTAAAGAACGTG } \\
\text { TGCATTA }\end{array}$ & 35 \\
\hline $21[175]$ & $19[181]$ & $\begin{array}{l}\text { TCATAACCCTCGTTAACGTAACAAAGCTC } \\
\text { CATGTT }\end{array}$ & 35 \\
\hline $21[238]$ & $19[244]$ & $\begin{array}{l}\text { GTTTTGCCAGAGGGATAGGCTGGCTGAC } \\
\text { TTGAAAG }\end{array}$ & 35 \\
\hline $21[217]$ & $19[223]$ & $\begin{array}{l}\text { CGAGAGGCTTTTGCAAGAGTAATCTTGA } \\
\text { AGGGAAC }\end{array}$ & 35 \\
\hline $1[189]$ & $29[195]$ & $\begin{array}{l}\text { GCGCCGCTACAGGGATAAATCAAAAGAA } \\
\text { TTTCTTT }\end{array}$ & 35 \\
\hline $11[140]$ & $9[146]$ & $\begin{array}{l}\text { TAGACGGGAGAATTAACCTCCCGACTTG } \\
\text { CAACAAT }\end{array}$ & 35 \\
\hline $1[63]$ & $29[69]$ & $\begin{array}{l}\text { GCTTGACGGGGAAACATCACCCAAATCA } \\
\text { AACTCAC }\end{array}$ & 35 \\
\hline $11[77]$ & $9[83]$ & $\begin{array}{l}\text { TAACGTCAAAAATGGCTACAATTTTATCT } \\
\text { AATTCT }\end{array}$ & 35 \\
\hline $1[147]$ & $29[153]$ & $\begin{array}{l}\text { GCGGTCACGCTGCGCAGTTTGGAACAAG } \\
\text { GCGCGGG }\end{array}$ & 35 \\
\hline $11[203]$ & $9[209]$ & $\begin{array}{l}\text { AGAGATAACCCACAATAGCAAGCAAATC } \\
\text { ACCAATC }\end{array}$ & 35 \\
\hline $21[70]$ & $19[76]$ & $\begin{array}{l}\text { TTACAGGTAGAAAGTGTGAATTACCTTA } \\
\text { AAAACAC }\end{array}$ & 35 \\
\hline $21[133]$ & $19[139]$ & TAACGCCAAAAGGAACGAGAAACACCAG & 35 \\
\hline
\end{tabular}




\begin{tabular}{|c|c|c|c|}
\hline & & GTATCAT & \\
\hline $11[98]$ & $9[104]$ & $\begin{array}{l}\text { CAGCCTTTACAGAGTTAGTTGCTATTTTA } \\
\text { ATAAAC }\end{array}$ & 35 \\
\hline $1[84]$ & $29[90]$ & $\begin{array}{l}\text { AACGTGGCGAGAAATCTATCACCCACTA } \\
\text { GCTCACT }\end{array}$ & 35 \\
\hline $21[154]$ & $19[160]$ & $\begin{array}{l}\text { GGCATAGTAAGAGCCAGTGAATAAGGCT } \\
\text { TGTCGAA }\end{array}$ & 35 \\
\hline $1[105]$ & $29[111]$ & $\begin{array}{l}\text { AAGAAAGCGAAAGGACGTCAAAGGGCG } \\
\text { ACGGGAAA }\end{array}$ & 35 \\
\hline $1[168]$ & $29[174]$ & $\begin{array}{l}\text { ACCACACCCGCCGCAGATAGGGTTGAGT } \\
\text { GTATTGG }\end{array}$ & 35 \\
\hline $11[161]$ & $9[167]$ & $\begin{array}{l}\text { CACCCTGAACAAAGAGAACGCGAGGCGT } \\
\text { CAAGAAA }\end{array}$ & 35 \\
\hline $11[119]$ & $9[125]$ & $\begin{array}{l}\text { CATAAAAACAGGGATTTTGAAGCCTTAA } \\
\text { ATGCAGA }\end{array}$ & 35 \\
\hline
\end{tabular}

Table S3. Sequences of DNA staples for tCRRO generated by cadnano software.

\begin{tabular}{|c|c|c|c|c|}
\hline Start & End & Sequence 5'-3, & Length & Color \\
\hline $20[239]$ & $18[240]$ & $\begin{array}{l}\text { TGAGGAAGAACATTATCATTTTGCTATACT } \\
\text { TC }\end{array}$ & 32 & \\
\hline $7[32]$ & $9[31]$ & TTTCAACAACCCTCGTTTACCATTTGCAAA & 30 & \\
\hline $21[96]$ & $23[95]$ & $\begin{array}{l}\text { GAAACCTGGGCAACAGCTGATTGCGAACA } \\
\text { AGA }\end{array}$ & 32 & \\
\hline $17[32]$ & $19[31]$ & $\begin{array}{l}\text { ATAATTCTGGTGCCGGAAACCAACTGTTG } \\
\text { G }\end{array}$ & 30 & \\
\hline $18[207]$ & $16[208]$ & $\begin{array}{l}\text { CAAAATTACAATAACGGATTCGCCATAAA } \\
\text { TCA }\end{array}$ & 32 & \\
\hline $8[47]$ & $6[48]$ & $\begin{array}{l}\text { ACTATCATTTTAATCATTGTGAATATTCAG } \\
\text { TG }\end{array}$ & 32 & \\
\hline
\end{tabular}




\begin{tabular}{|c|c|c|c|}
\hline $14[143]$ & $13[159]$ & $\begin{array}{l}\text { TTGCGGGAAAACATTATGACCCTGCGGAA } \\
\text { TCA }\end{array}$ & 32 \\
\hline $13[224]$ & $15[223]$ & CAACGCTATTTCATCTTCTGACATTTATCA & 30 \\
\hline $11[64]$ & $13[63]$ & $\begin{array}{l}\text { TGATAAGAAGATACATTTCGCAAAAATCA } \\
\text { TAC }\end{array}$ & 32 \\
\hline $16[143]$ & $15[159]$ & $\begin{array}{l}\text { CAATCATAACGGTAATCGTAAAACCCTTA } \\
\text { GAA }\end{array}$ & 32 \\
\hline $11[96]$ & $13[95]$ & $\begin{array}{l}\text { AGCTTAATTCTGCGAACGAGTAGAATTAA } \\
\text { GCA }\end{array}$ & 32 \\
\hline $16[175]$ & $14[176]$ & $\begin{array}{l}\text { AATCGTCGAACATAGCGATAGCTGATAAA } \\
\mathrm{T}\end{array}$ & 30 \\
\hline $11[32]$ & $13[31]$ & AGGATTATAGCTATATTTTCATCTACTAAT & 30 \\
\hline $20[47]$ & $18[48]$ & $\begin{array}{l}\text { TTGTTATCGATCGGTGCGGGCCTCTTTCCG } \\
\text { GC }\end{array}$ & 32 \\
\hline $8[271]$ & $6[272]$ & $\begin{array}{l}\text { AACATAAACAATAATAACGGAATATTACG } \\
\text { C }\end{array}$ & 30 \\
\hline $1[192]$ & $3[191]$ & $\begin{array}{l}\text { GGTCAGTGAGCGCAGTCTCTGAATATCAC } \\
\text { CGG }\end{array}$ & 32 \\
\hline $21[256]$ & $23[255]$ & $\begin{array}{l}\text { AAATGAAACCAACAGAGATAGAACCGCTC } \\
\text { AAT }\end{array}$ & 32 \\
\hline $13[120]$ & $15[127]$ & $\begin{array}{l}\text { AATCGGTTGTACCAAGAAGCCTTTATTTCA } \\
\text { CAAGAGAA }\end{array}$ & 38 \\
\hline $18[175]$ & $16[176]$ & $\begin{array}{l}\text { GAAATTGCACAGTAACAGTACCTTTCTGT } \\
\text { A }\end{array}$ & 30 \\
\hline $8[79]$ & $6[80]$ & $\begin{array}{l}\text { CAAAAGGAAAGAACTGGCTCATTCATTAC } \\
\text { C }\end{array}$ & 30 \\
\hline $22[47]$ & $20[48]$ & $\begin{array}{l}\text { CCACGCTGTGAGTGAGCTAACTCAGTGTG } \\
\text { AAA }\end{array}$ & 32 \\
\hline $6[271]$ & $4[272]$ & $\begin{array}{l}\text { AGTATGTTTGGGAATTAGAGCCAAAGGCC } \\
\text { G }\end{array}$ & 30 \\
\hline
\end{tabular}




\begin{tabular}{|c|c|c|c|}
\hline $7[224]$ & $9[223]$ & $\begin{array}{l}\text { ATAGCCGTGAACACCCTGAACACAGTTAC } \\
\text { A }\end{array}$ & 30 \\
\hline $10[207]$ & $8[208]$ & $\begin{array}{l}\text { CCGACTTGCCAGAGCCTAATTTGCAAGTC } \\
\text { AGA }\end{array}$ & 32 \\
\hline $21[32]$ & $23[31]$ & $\begin{array}{l}\text { TGCCTAAGTTTGCCCCAGCAGGGGCAAAA } \\
\mathrm{T}\end{array}$ & 30 \\
\hline $8[143]$ & $7[159]$ & $\begin{array}{l}\text { TAGAAAGACTAACGGAACAACATTATAAT } \\
\text { AAG }\end{array}$ & 32 \\
\hline $5[32]$ & $7[31]$ & $\begin{array}{l}\text { ATAAATTTTGCCCTGACGAGAATGGTTTA } \\
\text { A }\end{array}$ & 30 \\
\hline $9[64]$ & $11[63]$ & $\begin{array}{l}\text { TGTTTAGAATTAAGAGGAAGCCCGGCTCC } \\
\text { TTT }\end{array}$ & 32 \\
\hline $3[224]$ & $5[223]$ & $\begin{array}{l}\text { GAGCCGCCAGTAGCGACAGAATAATTATT } \\
\text { C }\end{array}$ & 30 \\
\hline $4[207]$ & $2[208]$ & $\begin{array}{l}\text { CCTTTAGCCCACCACCGGAACCGCTAAAG } \\
\text { CCA }\end{array}$ & 32 \\
\hline $2[143]$ & $1[159]$ & $\begin{array}{l}\text { ACGTTGAAGGAATTGCGAATAATATTGAT } \\
\text { GAT }\end{array}$ & 32 \\
\hline $9[96]$ & $11[95]$ & $\begin{array}{l}\text { AATCGTCAAGTCAGAAGCAAAGCGTGGCT } \\
\text { TAG }\end{array}$ & 32 \\
\hline $23[32]$ & $22[48]$ & $\begin{array}{l}\text { CCCTTATAAATCAAAAGAATAGCCAAGCG } \\
\text { GT }\end{array}$ & 31 \\
\hline $6[111]$ & $4[112]$ & $\begin{array}{l}\text { TAATCTTGAAGGGAACCGAACTGAATACG } \\
\text { TAA }\end{array}$ & 32 \\
\hline $10[191]$ & $12[184]$ & $\begin{array}{l}\text { TTTGAAGCTAGAAACCAATCAATATGCAG } \\
\text { AACGCGCCTGT }\end{array}$ & 40 \\
\hline $19[96]$ & $21[95]$ & $\begin{array}{l}\text { AAGGCGATCCCGGGTACCGAGCTCCCAGT } \\
\text { CGG }\end{array}$ & 32 \\
\hline $4[175]$ & $2[176]$ & CGGCATTTCTTTTCATAATCAAATTACCGT & 30 \\
\hline $2[111]$ & $0[112]$ & $\begin{array}{l}\text { AAGGAGCCAGCGGAGTGAGAATAGACCCT } \\
\text { CAT }\end{array}$ & 32 \\
\hline
\end{tabular}




\begin{tabular}{|c|c|c|c|}
\hline $6[143]$ & $5[159]$ & $\begin{array}{l}\text { GGCGCATAGACAGATGAACGGTGTCAGCG } \\
\text { CCA }\end{array}$ & 32 \\
\hline $16[47]$ & $14[48]$ & $\begin{array}{l}\text { AAAATTCGAAATTAATGCCGGAGAGGTAA } \\
\text { AGA }\end{array}$ & 32 \\
\hline $1[160]$ & $2[144]$ & $\begin{array}{l}\text { ACAGGAGTGCGTCATACATGGCTTATTTTT } \\
\text { TC }\end{array}$ & 32 \\
\hline $10[239]$ & $8[240]$ & $\begin{array}{l}\text { TCTAAGAACAGCCATATTATTTATAGACG } \\
\text { GGA }\end{array}$ & 32 \\
\hline $0[79]$ & $1[63]$ & $\begin{array}{l}\text { TGTACCGTAACACTGAGTTTCGTCAATGA } \\
\text { ATT }\end{array}$ & 32 \\
\hline $19[160]$ & $20[144]$ & $\begin{array}{l}\text { ACTTTACACATTTGAGGATTTAGAACGGC } \\
\text { CAG }\end{array}$ & 32 \\
\hline $9[224]$ & $11[223]$ & $\begin{array}{l}\text { AAATAAACGCGAGGCGTTTTAGTATCATT } \\
\text { C }\end{array}$ & 30 \\
\hline $1[32]$ & $3[31]$ & TGTCGTCAGTTGCGCCGACAATATTCGGTC & 30 \\
\hline $8[111]$ & $6[112]$ & $\begin{array}{l}\text { ACCACATTTGGGAAGAAAAATCTAATCAA } \\
\text { GAG }\end{array}$ & 32 \\
\hline $16[239]$ & $14[240]$ & $\begin{array}{l}\text { ACCTTTTTAGGTCTGAGAGACTACAAATAT } \\
\text { AT }\end{array}$ & 32 \\
\hline $1[256]$ & $3[263]$ & $\begin{array}{l}\text { TATTATTCCAGGTCAGACGATTGGACCCTC } \\
\text { AGAGCCACCA }\end{array}$ & 40 \\
\hline $11[256]$ & $13[255]$ & $\begin{array}{l}\text { CATCGAGAAGTACCGACAAAAGGTTTGAG } \\
\text { AAT }\end{array}$ & 32 \\
\hline $15[224]$ & $17[223]$ & $\begin{array}{l}\text { AAATCATTAATGGAAACAGTACTGATTGC } \\
\mathrm{T}\end{array}$ & 30 \\
\hline $12[207]$ & $10[208]$ & $\begin{array}{l}\text { TCAGCTAAATCGGCTGTCTTTCCTCGAACC } \\
\text { TC }\end{array}$ & 32 \\
\hline $12[239]$ & $10[240]$ & $\begin{array}{l}\text { TCTGTCCAGGGTATTAAACCAAGTTCCGGT } \\
\text { AT }\end{array}$ & 32 \\
\hline $16[63]$ & $18[56]$ & $\begin{array}{l}\text { ACGTTAATTAGCCAGCTTTCATCATCGCAC } \\
\text { TCCAGCCAGC }\end{array}$ & 40 \\
\hline
\end{tabular}




\begin{tabular}{|c|c|c|c|}
\hline $22[111]$ & $20[112]$ & $\begin{array}{l}\text { GTGAGACGTCGTGCCAGCTGCATTTCGAC } \\
\text { TCT }\end{array}$ & 32 \\
\hline $21[128]$ & $23[127]$ & GGCCAACCAGGGTGGTTTTTCTCCAACGTC & 30 \\
\hline $20[79]$ & $18[80]$ & $\begin{array}{l}\text { TAATCATGCTGGCGAAAGGGGGATCGGCC } \\
\mathrm{T}\end{array}$ & 30 \\
\hline $1[64]$ & $3[71]$ & $\begin{array}{l}\text { TTCTGTATGAGGTGAATTTCTTAAGGCCGC } \\
\text { TTTTGCGGGA }\end{array}$ & 40 \\
\hline $23[192]$ & $22[208]$ & $\begin{array}{l}\text { ATTACCGCCAGCCATTGCAACAGGCACAG } \\
\text { ACA }\end{array}$ & 32 \\
\hline $14[175]$ & $12[176]$ & $\begin{array}{l}\text { AAGGCGTTAGAAAAAGCCTGTTTTTATCA } \\
\text { A }\end{array}$ & 30 \\
\hline $0[207]$ & $1[191]$ & $\begin{array}{l}\text { GGAATAGGTGTATCACCGTACTCATTTAA } \\
\text { CGG }\end{array}$ & 32 \\
\hline $16[271]$ & $14[272]$ & $\begin{array}{l}\text { AATTAATTTTAGGTTGGGTTATACGCAAG } \\
\text { A }\end{array}$ & 30 \\
\hline $12[271]$ & $10[272]$ & $\begin{array}{l}\text { GAATATAAACAAGCAAGCCGTTTAATAGC } \\
\text { A }\end{array}$ & 30 \\
\hline $10[127]$ & $12[120]$ & $\begin{array}{l}\text { TCAGGTCTGTAGCTCAACATGTTGTTTCAT } \\
\text { TCCATATA }\end{array}$ & 38 \\
\hline $23[128]$ & $23[159]$ & $\begin{array}{l}\text { AAAGGGCGAAAAACCGTCTATCAACTCAA } \\
\text { AC }\end{array}$ & 31 \\
\hline $14[207]$ & $12[208]$ & $\begin{array}{l}\text { AATGGTTTTACAAATTCTTACCAGCAACAT } \\
\text { GT }\end{array}$ & 32 \\
\hline $7[128]$ & $9[135]$ & $\begin{array}{l}\text { AAACGAATTCATCAGTTGAGATCCCCCTC } \\
\text { AAATGCTT }\end{array}$ & 37 \\
\hline $10[175]$ & $8[176]$ & $\begin{array}{l}\text { CAAGATTACTGAATCTTACCAACGAGATA } \\
\text { A }\end{array}$ & 30 \\
\hline $18[79]$ & $16[80]$ & $\begin{array}{l}\text { CAGGAAGAACATTAAATGTGAGCATAAGC } \\
\text { A }\end{array}$ & 30 \\
\hline $16[255]$ & $18[248]$ & $\begin{array}{l}\text { CAATTTCACGCAGAGGCGAATTATTCCTG } \\
\text { ATTGTTTGGAT }\end{array}$ & 40 \\
\hline
\end{tabular}




\begin{tabular}{|c|c|c|c|}
\hline $5[192]$ & $7[191]$ & $\begin{array}{l}\text { GGAGGGAAGAATAAGTTTATTTTGTAGCT } \\
\text { ATC }\end{array}$ & 32 \\
\hline $14[111]$ & $12[112]$ & $\begin{array}{l}\text { ATAAAAATTCAGAGCATAAAGCTAACAGT } \\
\text { TGA }\end{array}$ & 32 \\
\hline $3[96]$ & $5[95]$ & $\begin{array}{l}\text { CAGCATCGCGAAGGCACCAACCTAGCAGA } \\
\text { CGG }\end{array}$ & 32 \\
\hline $7[56]$ & $9[63]$ & $\begin{array}{l}\text { TACCTTATGCGATTTTATTACGAGGCATAG } \\
\text { TATAGTAAAA }\end{array}$ & 40 \\
\hline $5[224]$ & $7[223]$ & $\begin{array}{l}\text { ATTAAAGCAACATATAAAAGAAGTAAGCA } \\
\text { G }\end{array}$ & 30 \\
\hline $18[47]$ & $16[48]$ & $\begin{array}{l}\text { ACCGCTTCGCGTCTGGCCTTCCTGATTTTG } \\
\text { TT }\end{array}$ & 32 \\
\hline $7[96]$ & $9[95]$ & $\begin{array}{l}\text { CAGGACGTCAACTAATGCAGATACTACTG } \\
\text { CGG }\end{array}$ & 32 \\
\hline $18[271]$ & $16[272]$ & $\begin{array}{l}\text { CAATATAATCATTTCAATTACCTAAAACA } \\
\text { A }\end{array}$ & 30 \\
\hline $15[96]$ & $17[95]$ & $\begin{array}{l}\text { CATTGCCTAAAACAGGAAGATTGTGAGTA } \\
\text { ACA }\end{array}$ & 32 \\
\hline $12[47]$ & $10[48]$ & $\begin{array}{l}\text { AACCTGTTGAGAGTACCTTTAATTAAAGA } \\
\text { CTT }\end{array}$ & 32 \\
\hline $20[175]$ & $18[176]$ & $\begin{array}{l}\text { AGATAATAAACAATTCGACAACTAAATAA } \\
\text { A }\end{array}$ & 30 \\
\hline $19[32]$ & $21[31]$ & $\begin{array}{l}\text { GAAGGGCCGCTCACAATTCCACGCCTGGG } \\
\text { G }\end{array}$ & 30 \\
\hline $8[175]$ & $6[176]$ & $\begin{array}{l}\text { CCCACAAGACAATGAAATAGCAATCACAA } \\
\mathrm{T}\end{array}$ & 30 \\
\hline $10[271]$ & $8[272]$ & AGCAAATCAACGATTTTTTGTTTAGAGAAT & 30 \\
\hline $14[79]$ & $12[80]$ & $\begin{array}{l}\text { AATGCAATCAAAGAATTAGCAAATTTAGT } \\
\mathrm{T}\end{array}$ & 30 \\
\hline $22[143]$ & $21[159]$ & $\begin{array}{l}\text { TTGGGCGCGCGCGGGGAGAGGCGGGCCAT } \\
\text { TAA }\end{array}$ & 32 \\
\hline
\end{tabular}




\begin{tabular}{|c|c|c|c|}
\hline $13[160]$ & $14[144]$ & $\begin{array}{l}\text { TAATTACTAAATAAGAATAAACACTAATA } \\
\text { CTT }\end{array}$ & 32 \\
\hline $23[64]$ & $22[80]$ & GGGTTGAGTGTTGTTCCAGTTTGCCTTCAC & 30 \\
\hline $4[255]$ & $6[248]$ & $\begin{array}{l}\text { AACCATCGACCGACTTGAGCCATTAGCAA } \\
\text { ACGTAGAAAAT }\end{array}$ & 40 \\
\hline $19[248]$ & $21[255]$ & $\begin{array}{l}\text { GGAACAAAGAAACCACGGCAAATCAACA } \\
\text { GTTGCCAGCAGC }\end{array}$ & 40 \\
\hline $4[111]$ & $2[112]$ & $\begin{array}{l}\text { TGCCACTAGAACGAGGGTAGCAACGGCTC } \\
\text { CAA }\end{array}$ & 32 \\
\hline $23[224]$ & $22[240]$ & $\begin{array}{l}\text { TCATGGAAATACCTACATTTTGACCTTCTG } \\
\text { A }\end{array}$ & 31 \\
\hline $3[128]$ & $5[127]$ & $\begin{array}{l}\text { AGGCTTTCATTAAACGGGTAAACCAACTT } \\
\mathrm{T}\end{array}$ & 30 \\
\hline $15[128]$ & $17[127]$ & $\begin{array}{l}\text { TCGATGATGTACCCCGGTTGATAAACGGC } \\
\text { G }\end{array}$ & 30 \\
\hline $6[175]$ & $4[176]$ & $\begin{array}{l}\text { CAATAGAAAGGGCGACATTCAACTTTTCA } \\
\mathrm{T}\end{array}$ & 30 \\
\hline $2[239]$ & $0[240]$ & $\begin{array}{l}\text { TTCACAAAATGCCCCCTGCCTATTGGATAA } \\
\text { GT }\end{array}$ & 32 \\
\hline $14[271]$ & $12[272]$ & $\begin{array}{l}\text { CAAAGAACTTAACAACGCCAACAAATAAG } \\
\text { A }\end{array}$ & 30 \\
\hline $20[111]$ & $18[112]$ & $\begin{array}{l}\text { AGAGGATCTAAGTTGGGTAACGCCGCATC } \\
\text { TGC }\end{array}$ & 32 \\
\hline $20[207]$ & $18[208]$ & $\begin{array}{l}\text { CACTAACACCCGAACGTTATTAATTACCAT } \\
\text { AT }\end{array}$ & 32 \\
\hline $7[248]$ & $9[255]$ & $\begin{array}{l}\text { GGAAACCGAGGAAACGAACAGGGAAGCG } \\
\text { CATTCCCAATCC }\end{array}$ & 40 \\
\hline $17[160]$ & $18[144]$ & $\begin{array}{l}\text { ATGAATATGTAGATTTTCAGGTTTACGTTG } \\
\text { GT }\end{array}$ & 32 \\
\hline $22[239]$ & $20[240]$ & $\begin{array}{l}\text { CCTGAAAGAGTGCCACGCTGAGAGAAAGG } \\
\text { AAT }\end{array}$ & 32 \\
\hline
\end{tabular}




\begin{tabular}{|c|c|c|c|}
\hline $8[207]$ & $6[208]$ & $\begin{array}{l}\text { GGGTAATTGCCCTTTTTAAGAAAAACGCA } \\
\text { AAG }\end{array}$ & 32 \\
\hline $23[256]$ & $22[272]$ & $\begin{array}{l}\text { CGTCTGAAATGGATTATTTACATAAAGGG } \\
\text { A }\end{array}$ & 30 \\
\hline $18[111]$ & $16[112]$ & $\begin{array}{l}\text { CAGTTTGAGATTCTCCGTGGGAACAATCA } \\
\text { GAA }\end{array}$ & 32 \\
\hline $14[239]$ & $12[240]$ & $\begin{array}{l}\text { TTTAGTTACAACAGTAGGGCTTAAAAAGT } \\
\text { AAT }\end{array}$ & 32 \\
\hline $10[47]$ & $8[48]$ & $\begin{array}{l}\text { CAAATATCTTGCCAGAGGGGGTAAAGAGC } \\
\text { AAC }\end{array}$ & 32 \\
\hline $2[175]$ & $0[176]$ & $\begin{array}{l}\text { TCCAGTAAGTACTGGTAATAAGTGGAGGT } \\
\mathrm{T}\end{array}$ & 30 \\
\hline $6[207]$ & $4[208]$ & $\begin{array}{l}\text { ACACCACGGGTAAATATTGACGGACAAGT } \\
\text { TTG }\end{array}$ & 32 \\
\hline $23[96]$ & $22[112]$ & $\begin{array}{l}\text { GTCCACTATTAAAGAACGTGGACTTTTCAC } \\
\text { CA }\end{array}$ & 32 \\
\hline $10[79]$ & $8[80]$ & $\begin{array}{l}\text { TCAAAAAGCTGGATAGCGTCCAAATAACG } \\
\text { C }\end{array}$ & 30 \\
\hline $2[47]$ & $0[48]$ & $\begin{array}{l}\text { ATACCGATTTTCCAGACGTTAGTAACCAGT } \\
\text { AC }\end{array}$ & 32 \\
\hline $0[239]$ & $1[223]$ & $\begin{array}{l}\text { GCCGTCGAGAGGGTTGATATAAGCCGTAT } \\
\text { AA }\end{array}$ & 31 \\
\hline $6[239]$ & $4[240]$ & $\begin{array}{l}\text { AAAGGTGGGTGAATTATCACCGTCATAGC } \\
\text { AGC }\end{array}$ & 32 \\
\hline $16[79]$ & $14[80]$ & AATATTTAGAGATCTACAAAGGCTATTTTA & 30 \\
\hline $4[79]$ & $2[80]$ & $\begin{array}{l}\text { AGAGGCAATCGTCACCCTCAGCATATCAG } \\
\text { C }\end{array}$ & 30 \\
\hline $21[160]$ & $22[144]$ & $\begin{array}{l}\text { AAATACCGATAGCCCTAAAACATCTTTGC } \\
\text { GTA }\end{array}$ & 32 \\
\hline $12[79]$ & $10[80]$ & TGACCATTGGTCATTTTTGCGGAGATTGCA & 30 \\
\hline
\end{tabular}




\begin{tabular}{|c|c|c|c|}
\hline $22[207]$ & $20[208]$ & $\begin{array}{l}\text { ATATTTTTTGAGGCGGTCAGTATTTTTAGG } \\
\text { AG }\end{array}$ & 32 \\
\hline $17[128]$ & $19[127]$ & $\begin{array}{l}\text { GATTGACGCGCATCGTAACCGTAGGGTTT } \\
\mathrm{T}\end{array}$ & 30 \\
\hline $23[160]$ & $22[176]$ & TATCGGCCTTGCTGGTAATATCCTTAATGC & 30 \\
\hline $1[128]$ & $3[127]$ & $\begin{array}{l}\text { AACTAAAAATCTCCAAAAAAAAGGCTACA } \\
\text { G }\end{array}$ & 30 \\
\hline $2[271]$ & $0[272]$ & $\begin{array}{l}\text { GGTTGAGGTGAAACATGAAAGTAAGGATT } \\
\text { A }\end{array}$ & 30 \\
\hline $0[47]$ & $1[31]$ & $\begin{array}{l}\text { AAACTACAACGCCTGTAGCATTCTAAAGT } \\
\text { TT }\end{array}$ & 31 \\
\hline $9[256]$ & $11[255]$ & $\begin{array}{l}\text { AAATAAGAAGATATAGAAGGCTTAACCGC } \\
\text { ACT }\end{array}$ & 32 \\
\hline $4[47]$ & $2[48]$ & $\begin{array}{l}\text { TTGACCCCCTTGCAGGGAGTTAAAACAGC } \\
\text { TTG }\end{array}$ & 32 \\
\hline $17[192]$ & $19[191]$ & $\begin{array}{l}\text { GGGAGAAATTTGCACGTAAAACAGCGTAT } \\
\text { TAA }\end{array}$ & 32 \\
\hline $14[47]$ & $12[48]$ & $\begin{array}{l}\text { TTCAAAAGGCATTAACATCCAATATGGTC } \\
\text { AAT }\end{array}$ & 32 \\
\hline $1[224]$ & $3[223]$ & ACAGTTACAAATAAАTCCTCATCTCССТCA & 30 \\
\hline $5[96]$ & $7[95]$ & $\begin{array}{l}\text { TCAATCATACAAGAACCGGATATTATACC } \\
\text { AGT }\end{array}$ & 32 \\
\hline $22[79]$ & $20[80]$ & CGCCTGGCCTCACTGCCCGCTTTGAATTCG & 30 \\
\hline $21[192]$ & $23[191]$ & $\begin{array}{l}\text { AACAGAGGGAATGGCTATTAGTCTAGAAC } \\
\text { AAT }\end{array}$ & 32 \\
\hline $20[271]$ & $18[272]$ & $\begin{array}{l}\text { GGTCAGTTCAGAAGGAGCGGAATAATTCA } \\
\mathrm{T}\end{array}$ & 30 \\
\hline $18[143]$ & $17[159]$ & $\begin{array}{l}\text { GTAGATGGCGTAATGGGATAGGTCAACGT } \\
\text { CAG }\end{array}$ & 32 \\
\hline $0[143]$ & $1[127]$ & GAACCGCCACCCTCAGAGCCACCAAAGGA & 31 \\
\hline
\end{tabular}




\begin{tabular}{|c|c|c|c|}
\hline & & $\mathrm{AC}$ & \\
\hline $13[32]$ & $15[31]$ & $\begin{array}{l}\text { AGTAGTAGGTGAGAAAGGCCGGACCGTTC } \\
\mathrm{T}\end{array}$ & 30 \\
\hline $21[64]$ & $23[63]$ & $\begin{array}{l}\text { GCGTTGCGCCTGAGAGAGTTGCAGCCGAG } \\
\text { ATA }\end{array}$ & 32 \\
\hline $1[96]$ & $3[95]$ & $\begin{array}{l}\text { ACAGTTTCTTTAATTGTATCGGTTGCGAAA } \\
\text { GA }\end{array}$ & 32 \\
\hline $22[271]$ & $20[272]$ & CATTCTGGAATCTAAAGCATCACAATATCT & 30 \\
\hline $13[256]$ & $15[263]$ & $\begin{array}{l}\text { CGCCATATGCGAGAAAACTTTTTCCTTTTT } \\
\text { AACCTCCGGC }\end{array}$ & 40 \\
\hline $11[224]$ & $13[223]$ & $\begin{array}{l}\text { CAAGAACGACGACGACAATAAATATAAA } \\
\text { GC }\end{array}$ & 30 \\
\hline $16[111]$ & $14[112]$ & $\begin{array}{l}\text { AAGCCCCAGAGAGTCTGGAGCAAAACGCA } \\
\text { AGG }\end{array}$ & 32 \\
\hline $9[160]$ & $10[144]$ & $\begin{array}{l}\text { ATTTTATCGTTGCTATTTTGCACCCGAGAA } \\
\text { TG }\end{array}$ & 32 \\
\hline $10[111]$ & $8[112]$ & $\begin{array}{l}\text { ACTATTATTAAATATTCATTGAATTTAGGA } \\
\text { AT }\end{array}$ & 32 \\
\hline $19[224]$ & $21[223]$ & TTTGAGTGTTATCTAAAATATCAACACCGC & 30 \\
\hline $2[207]$ & $0[208]$ & $\begin{array}{l}\text { GAATGGAACCTTGAGTAACAGTGCTATAG } \\
\text { CCC }\end{array}$ & 32 \\
\hline $12[143]$ & $11[159]$ & $\begin{array}{l}\text { GTCTGGAATTAAATATGCAACTAAAAATA } \\
\text { ATA }\end{array}$ & 32 \\
\hline $21[224]$ & $23[223]$ & $\begin{array}{l}\text { CTGCAACCGTAAGAATACGTGGAAAAACG } \\
\text { C }\end{array}$ & 30 \\
\hline $13[64]$ & $15[71]$ & $\begin{array}{l}\text { AGGCAAGGGCCTGAGTAATGTGTAGGGTA } \\
\text { GCTATTTTTGA }\end{array}$ & 40 \\
\hline $22[175]$ & $20[176]$ & $\begin{array}{l}\text { GCGAACTGAACGAACCACCAGCACGTCAA } \\
\mathrm{T}\end{array}$ & 30 \\
\hline $3[192]$ & $5[191]$ & AACCAGAGGTCAGACTGTAGCGCGCGATT & 32 \\
\hline
\end{tabular}




\begin{tabular}{|c|c|c|c|}
\hline & & GAG & \\
\hline $4[239]$ & $2[240]$ & $\begin{array}{l}\text { ACCGTAATCACCCTCAGAACCGCCCCTTG } \\
\text { ATA }\end{array}$ & 32 \\
\hline $5[128]$ & $7[127]$ & $\begin{array}{l}\text { GAAAGAGGGCTGGCTGACCTTCCGTTAAT } \\
\text { A }\end{array}$ & 30 \\
\hline $19[128]$ & $21[127]$ & $\begin{array}{l}\text { CCCAGTCTTGCATGCCTGCAGGAATGAAT } \\
\text { C }\end{array}$ & 30 \\
\hline $3[160]$ & $4[144]$ & $\begin{array}{l}\text { TTTGCCATTCGGTCATAGCCCCCTTTCATG } \\
\text { AG }\end{array}$ & 32 \\
\hline $8[239]$ & $6[240]$ & $\begin{array}{l}\text { GAATTAACAACAAAGTTACCAGAAACATA } \\
\text { CAT }\end{array}$ & 32 \\
\hline $15[160]$ & $16[144]$ & $\begin{array}{l}\text { TCCTTGAACTATTAATTAATTTTCTAGCAT } \\
\text { GT }\end{array}$ & 32 \\
\hline $12[175]$ & $10[176]$ & $\begin{array}{l}\text { CAATAGATTAATTTACGAGCATGCTTAAA } \\
\mathrm{T}\end{array}$ & 30 \\
\hline $11[160]$ & $12[144]$ & $\begin{array}{l}\text { TCCCATCCAAGTCCTGAACAAGAAAGTAC } \\
\text { GGT }\end{array}$ & 32 \\
\hline $0[271]$ & $1[255]$ & $\begin{array}{l}\text { GCGGGGTTTTGCTCAGTACCAGGCTCGGA } \\
\text { ACC }\end{array}$ & 32 \\
\hline $7[192]$ & $9[199]$ & $\begin{array}{l}\text { TTACCGAAGAGCGCTAATATCAGAGCTAA } \\
\text { CGAGCGTCTTT }\end{array}$ & 40 \\
\hline $12[111]$ & $10[112]$ & $\begin{array}{l}\text { TTCCCAATTGCTGAATATAATGCTTTACCC } \\
\text { TG }\end{array}$ & 32 \\
\hline 18 [239] & $16[240]$ & $\begin{array}{l}\text { TGAATAATCCAAGTTACAAAATCGTTTGA } \\
\text { ATT }\end{array}$ & 32 \\
\hline $10[143]$ & $9[159]$ & $\begin{array}{l}\text { ACCATAAATAAACAGTTCAGAAAACAGCT } \\
\text { ACA }\end{array}$ & 32 \\
\hline $7[160]$ & $8[144]$ & $\begin{array}{l}\text { AGCAAGAAAATTGAGTTAAGCCCAATTAC } \\
\text { AGG }\end{array}$ & 32 \\
\hline $19[56]$ & $21[63]$ & $\begin{array}{l}\text { TTCGCTATTACGCCAGGTCATAGCTGTTTC } \\
\text { CTCATTAATT }\end{array}$ & 40 \\
\hline
\end{tabular}




\begin{tabular}{|c|c|c|c|}
\hline $16[207]$ & $14[208]$ & $\begin{array}{l}\text { ATATATGTGAAGAGTCAATAGTGACTAAA } \\
\text { TTT }\end{array}$ & 32 \\
\hline $15[192]$ & $17[191]$ & $\begin{array}{l}\text { GACGCTGAGAGTGAATAACCTTGCTTTAC } \\
\text { ATC }\end{array}$ & 32 \\
\hline $20[143]$ & $19[159]$ & $\begin{array}{l}\text { TGCCAAGCACGACGTTGTAAAACGAGTAT } \\
\text { TAG }\end{array}$ & 32 \\
\hline $4[271]$ & $2[272]$ & $\begin{array}{l}\text { GAAACGTCCCCTCAGAGCCGCCAGACAGG } \\
\text { A }\end{array}$ & 30 \\
\hline $15[32]$ & $17[31]$ & AGCTGATCATTAAATTTTTGTTCATCAAAA & 30 \\
\hline $13[184]$ & $15[191]$ & $\begin{array}{l}\text { AGTATCATATGCGTTAGAAATACCGACCG } \\
\text { TGTTAGATTAA }\end{array}$ & 40 \\
\hline $17[96]$ & $19[95]$ & $\begin{array}{l}\text { ACCCGTCGGGGGACGACGACAGTATGTGC } \\
\text { TGC }\end{array}$ & 32 \\
\hline $6[79]$ & $4[80]$ & $\begin{array}{l}\text { CAAATCAATAGCCGGAACGAGGCAAACG } \\
\text { AA }\end{array}$ & 30 \\
\hline $2[79]$ & $0[80]$ & TTGCTTTCGGGATTTTGCTAAACGAACCCA & 30 \\
\hline $5[160]$ & $6[144]$ & $\begin{array}{l}\text { AAGACAAAAATTCATATGGTTTACACAGA } \\
\text { CCA }\end{array}$ & 32 \\
\hline $17[224]$ & $19[223]$ & $\begin{array}{l}\text { TTGAATAGGAAGGGTTAGAACCTTTAAAA } \\
\text { G }\end{array}$ & 30 \\
\hline $6[47]$ & $4[48]$ & $\begin{array}{l}\text { AATAAGGCGTGTCGAAATCCGCGAACTCA } \\
\text { TCT }\end{array}$ & 32 \\
\hline $0[175]$ & $0[144]$ & $\begin{array}{l}\text { TAGTACCGCCACCCTCAGAACCGCCACCC } \\
\text { TCA }\end{array}$ & 32 \\
\hline $9[32]$ & $11[31]$ & $\begin{array}{l}\text { AGAAGTTGCGTTTTAATTCGAGAACAGGT } \\
\text { C }\end{array}$ & 30 \\
\hline $0[111]$ & $1[95]$ & $\begin{array}{l}\text { TTTCAGGGATAGCAAGCCCAATAGAACTT } \\
\text { TCA }\end{array}$ & 32 \\
\hline $13[96]$ & $15[95]$ & $\begin{array}{l}\text { ATAAAGCCTTTTAGAACCCTCATATATCAG } \\
\text { GT }\end{array}$ & 32 \\
\hline
\end{tabular}




\begin{tabular}{|l|l|l|l|l|}
\hline $4[63]$ & $6[56]$ & $\begin{array}{l}\text { ACTAAAACCCTGCTCCATGTTACTCGTAAC } \\
\text { AAAGCTGCTC }\end{array}$ & 40 \\
\hline $19[192]$ & $21[191]$ & $\begin{array}{l}\text { ATCCTTTGACTAATAGATTAGAGCGAAGA } \\
\text { TAA }\end{array}$ & 32 \\
\hline $3[32]$ & $5[31]$ & $\begin{array}{l}\text { GCTGAGGCAGCGATTATACCAAATCGCCT } \\
\text { G }\end{array}$ & 30 \\
\hline $4[143]$ & $3[159]$ & $\begin{array}{l}\text { GAAGTTTCGAGGACTAAAGACTTTTATTA } \\
\text { GCG }\end{array}$ & 32 \\
\hline
\end{tabular}

\section{References}

(1) Rothemund, P. W. K. Nature 2006, 440, 297-302.

(2) Kopperger, E.; Pirzer, T.; Simmel, F. C. Nano Lett 2015, 15, 2693-2699.

(3) Martin, T. G.; Dietz, H. Nature Communications 2012, 3, 1103-6.

(4) Kick, B., Praetorius, F., Dietz, H., and Weuster-Botz, D. Nano Lett. 2015, 15, 4672-4676.

(5) Schindelin, J.; Arganda-Carreras, I.; Frise, E.; Kaynig, V.; Longair, M.; Pietzsch, T.; Preibisch, S.; Rueden, C.; Saalfeld, S.; Schmid, B.; Tinevez, J. Y.; White, D. J.; Hartenstein, V.; Eliceiri, K.; Tomancak, P.; Cardona, A. Nat Methods 2012, 9, 676-82.

(6) de la Rosa-Trevin, J. M.; Quintana, A.; del Cano, L.; Zaldívar, A.; Foche, I.; Gutiérrez, J.; Gómez-Blanco, J.; Burguet-Castell, J.; Cuenca-Alba, J.; Abrishami, V.; Vargas, J.; Otón, J.; Sharov, G.; Vilas, J. L.; Navas, J.; Conesa, P.; Kazemi, M.; Marabini, R.; Sorzano, C. O. S.; Carazo, J. M. J Struct Biol 2016, 195, 93-99.

(7) Stahl, E.; Martin, T. G.; Praetorius, F.; Dietz, H. Angew Chem Int Ed 2014, 53, 12735-12740.

(8) Shaw, A., Benson, E. and Högberg, B. ACS Nano 2015, 9, 4968-4975.

(9) Bellot, G.; Mcclintock, M. A.; Lin, C.; Shih, W. M. Nat Methods 2011, 8, 192-194.

(10) Raspaud, E., de la Cruz, M.O., Sikorav, J. L. and Livolant, F. Biophysical J 1998, 74, 381-393.

(11) Hahn, J., Wickham, S. F. J., Shih, W. M. and Perrault, S. D. ACS Nano 2014, 8, 8765-8775. 